\title{
Diabetes self-management : strategies to support patients and health care professionals
}

\author{
Citation for published version (APA): \\ Heinrich, E. (2011). Diabetes self-management : strategies to support patients and health care \\ professionals. [Doctoral Thesis, Maastricht University]. Universiteit Maastricht. \\ https://doi.org/10.26481/dis.20110218eh
}

Document status and date:

Published: 01/01/2011

DOI:

10.26481/dis.20110218eh

Document Version:

Publisher's PDF, also known as Version of record

\section{Please check the document version of this publication:}

- A submitted manuscript is the version of the article upon submission and before peer-review. There can be important differences between the submitted version and the official published version of record. People interested in the research are advised to contact the author for the final version of the publication, or visit the DOI to the publisher's website.

- The final author version and the galley proof are versions of the publication after peer review.

- The final published version features the final layout of the paper including the volume, issue and page numbers.

Link to publication

\footnotetext{
General rights rights.

- You may freely distribute the URL identifying the publication in the public portal. please follow below link for the End User Agreement:

www.umlib.nl/taverne-license

Take down policy

If you believe that this document breaches copyright please contact us at:

repository@maastrichtuniversity.nl

providing details and we will investigate your claim.
}

Copyright and moral rights for the publications made accessible in the public portal are retained by the authors and/or other copyright owners and it is a condition of accessing publications that users recognise and abide by the legal requirements associated with these

- Users may download and print one copy of any publication from the public portal for the purpose of private study or research.

- You may not further distribute the material or use it for any profit-making activity or commercial gain

If the publication is distributed under the terms of Article $25 \mathrm{fa}$ of the Dutch Copyright Act, indicated by the "Taverne" license above, 


\section{DIABETES SELF-MANAGEMENT}

Strategies to support patients and health care professionals 


\section{Colophon}

Cover: Kim van Bakel

Layout: Tiny Wouters

(C) Evelien Heinrich, Venlo, 2011

ISBN 978-90-9025909-3

The research presented in this thesis was conducted under the auspices of the School for Public Health and Primary Care (CAPHRI), which participates in the Netherlands School of Primary Care Research (CaRe), acknowledged in 1995 by the Royal Netherlands Academy of Arts and Sciences (KNAW).

The studies presented in this thesis were conducted with financial support from the Dr. Paul Janssen Foundation and from the Dutch Organisation for Health Research and Development (ZonMw), project number 76500004.

Financial support by LifeScan for printing this dissertation is gratefully acknowledged.

All rights are reserved. No part of this book may be reproduced or transmitted without written permission from the author or, when appropriate, the publisher of the article. 


\section{DIABETES SELF-MANAGEMENT}

Strategies to support patients and health care professionals

\section{PROEFSCHRIFT}

ter verkrijging van de graad van doctor aan de Universiteit Maastricht, op gezag van de Rector Magnificus, Prof. mr. G.P.M.F. Mols, volgens besluit van het College van Decanen, in het openbaar te verdedigen op vrijdag 18 februari 2011 om 14:00 uur

door

Evelien Heinrich 


\section{Promotores}

Prof. dr. N.K. de Vries

Prof. dr. N.C. Schaper

\section{Beoordelingscommissie}

Prof. dr. H.W. van den Borne (voorzitter)

Prof. dr. G.E.H.M. Rutten (Universitair Medisch Centrum Utrecht)

Prof. dr. F.J. Snoek (VU Medisch Centrum Amsterdam)

Prof. dr. C. Spreeuwenberg

Prof. dr. T. van der Weijden 


\section{Contents}

$\begin{array}{lll}\text { Chapter } 1 \text { General Introduction } & 7\end{array}$

Chapter 2 Development of the web-based type 2 diabetes 21 education programme: DIEP

Chapter 3 Evaluation of the web-based Diabetes Interactive 31 Education Programme (DIEP) for patients with type 2 diabetes

Chapter 4 Effect evaluation of a Motivational Interviewing based counselling strategy in diabetes care

Chapter 5 Motivational Interviewing based counselling in diabetes care: an implementation study among nurses

Chapter 6 Self-management interventions for type 2 diabetes: a systematic review

$\begin{array}{lll}\text { Chapter } 7 & \text { Summary and General Discussion } & 101\end{array}$

$\begin{array}{ll}\text { Samenvatting } & 127\end{array}$

$\begin{array}{ll}\text { References } & 133\end{array}$

$\begin{array}{ll}\text { Dankwoord } & 151\end{array}$

$\begin{array}{ll}\text { Curriculum Vitae } & 155\end{array}$ 



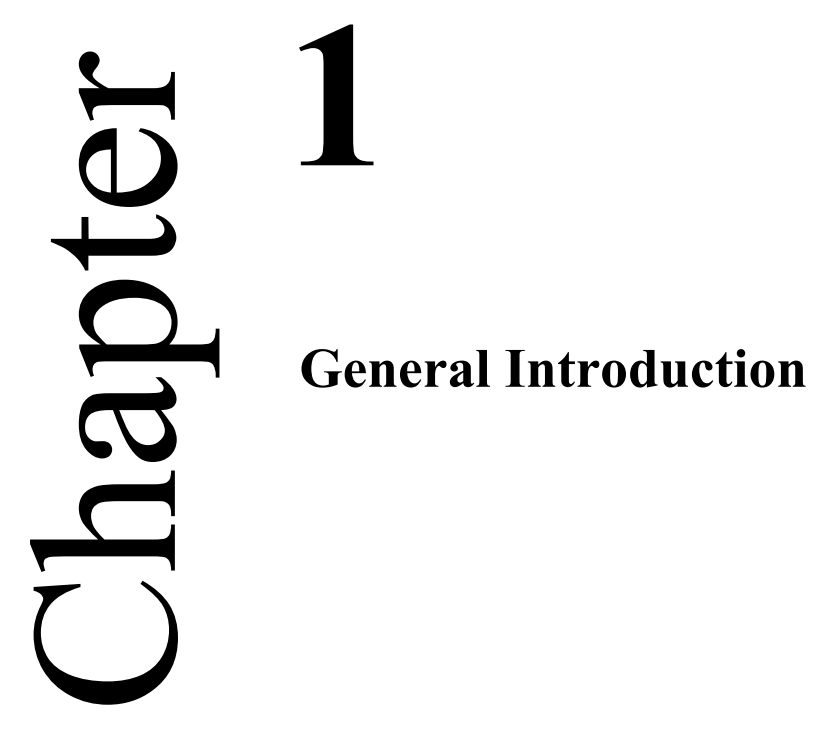




\section{INTRODUCTION}

Diabetes mellitus is a growing health threat for people all over the world. Especially the incidence of type 2 diabetes increases due to aging populations and changing lifestyles. To limit the consequences of this chronic disease for patients and society, effective treatment strategies are urgently needed. Besides medication, diabetes treatment relies heavily on patients' self-management capacities. However, patients experience difficulties with self-management, especially with maintaining behaviour change which is often necessary. Therefore, health care professionals need effective ways to support their patients.

This dissertation is about the development, content and evaluation of an education and counselling strategy aimed at both, patients and health care professionals, to support patients' self-management and thereby limit the impact of the disease. This chapter starts with background information about diabetes and the two most common types of diabetes, followed by a paragraph on self-management and a paragraph on diabetes care. Next, the aims of the current research project are given and strategies to improve self-management are described. The introduction ends with an outline of this dissertation.

\section{DIABETES MELLITUS}

Diabetes mellitus is a chronic disease which affects approximately 285 million people worldwide (International Diabetes Federation, 2010). This public health problem has reached pandemic proportions; a prevalence of 439 million people with diabetes in the year 2030 has been predicted (International Diabetes Federation, 2010). In the Netherlands, the estimated prevalence was 740.000 people with diabetes in 2007 , which was roughly $4.5 \%$ of the population (RIVM, 2010). Several factors are held responsible for the rise in diabetes; aging populations, dietary changes, and reduced physical activity (International Diabetes Federation, 2010). Also, health care professionals are nowadays more alert to risk factors of diabetes and therefore patients are earlier diagnosed by case finding than before.

Diabetes is an endocrine disorder which is characterized by a deficiency of and/or resistance to the hormone insulin. Insulin is produced in the beta-cells of the pancreas and is an important regulator of the glucose metabolism. Insulin is responsible for the uptake and storage of glucose from the blood and thereby reduces blood glucose levels. If the glucose metabolism is disturbed, 
hypoglycaemia (low blood glucose levels) or hyperglycaemia (high blood glucose levels) might occur. In addition, insulin plays an important role in fat and protein metabolism.

There are several types of diabetes, but the two most common types are type 1 and type 2 diabetes. Type 1 diabetes is caused by an auto-immune response leading to a breakdown of insulin producing cells. This results in an absolute insulin deficit and therefore, all patients with type 1 diabetes need daily injections of insulin in order to lower their blood glucose levels. This type of diabetes can affect people of any age, but usually already manifests in children or young adults and accounts for $10-15 \%$ of all people with diabetes. By contrast, type 2 diabetes has the fastest increasing incidence and accounts for approximately $85-90 \%$ of the diabetes population. The pathogenesis of type 2 diabetes differs from type 1 diabetes. Two key features are important, namely insulin resistance and beta-cell failure. Because of insulin resistance, body cells are less sensitive to the glucose-lowering effects of insulin, which in nondiabetic people is compensated by an increase in pancreatic insulin secretion. However, in people with type 2 diabetes the pancreas fails to sufficiently increase this secretion, resulting in a rise of the blood glucose levels. The pathogenesis of this progressive beta-cell failure is only partly unravelled; probably both genetic and metabolic factors are important.

Type 2 diabetes (DM2) (and insulin resistance) usually develops in the obese adult, and the risk for type 2 diabetes is closely associated with the body mass index (BMI). Conversely, weight loss clearly reduces the risk for DM2 in obese subjects and can result in a marked improvement of blood glucose levels in obese DM2 patients. Other factors associated with insulin resistance and the development of DM2 are physical inactivity, dietary intake, smoking, age, family history of diabetes and ethnicity. Besides hyperglycaemia, DM2 is clearly associated with other cardiovascular risk factors such as dyslipidemia and hypertension.

The most important parameter for glycaemic control is the percentage of glycated haemoglobin ( $\mathrm{HbAlc})$. The Hbalc-level is an estimate of the average plasma glucose concentration over a period of 6-8 weeks. Diabetes treatment is usually targeted at $\mathrm{HbA} 1 \mathrm{c}$-levels below $7 \%{ }^{1}$, depending on age, co-morbidity

\footnotetext{
${ }^{1}$ Before April 2010, in the Netherlands HbA1c-levels were expressed in \%. To facilitate international comparisons in HbAlc-levels, one international unit (mmol $/ \mathrm{mol}$ ) was chosen. An HbAlc-level of $7 \%=53 \mathrm{mmol} / \mathrm{mol}$. Our research was carried out before 2010. Therefore, in this dissertation HbAlc-levels are still reported in $\%$.
} 
and life expectancy. Levels above $8 \%$ indicate poor glycaemic control. Prolonged poor metabolic control increases the risk of developing complications of which the consequences are far-reaching. Microvascular complications are retinopathy, nephropathy, and neuropathy; these complications are closely associated with both the severity and the duration of hyperglycaemia (Stratton, Adler, Neil et al., 2000). Long-term lowering of elevated blood glucose levels decreases the risk for these complications (DCCT Research Group, 1993). Macrovascular complications are premature atherosclerotic vascular diseases; myocardial infarction, cerebrovascular accident, and peripheral vascular disease. These complications are mainly related to cardiovascular risk factors, with hyperglycaemia playing a secondary role. Diabetic complications often result in increased disability, reduced quality of life, reduced life expectancy and enormous health costs for society (International Diabetes Federation, 2010).

Although diabetes usually cannot be cured, complications can be prevented or delayed. The multifactorial treatment is targeted at achieving near normal glycaemia and minimizing other cardiovascular risk factors. Diabetes treatment mostly consists of lifestyle modification such as weight loss in obese patients, increasing physical activity, dietary changes and smoking cessation and of pharmacological treatment. In addition, patients with type 1 diabetes need insulin injections from the moment they are diagnosed with diabetes. Patients with type 2 diabetes are not dependent on exogenous insulin, unless lifestyle changes and oral hypoglycaemic agents are insufficient.

\section{SELF-MANAGEMENT}

The pharmacological treatment for patients with diabetes is highly advanced and previous studies have shown the beneficial effects of intensive glucose therapy on the risk of complications (Holman, Paul, Bethel et al., 2008; UK Prospective Diabetes Study Group, 1998). However, even more important than a prescription for medical treatment by health care professionals is the patients' key role in the management of their disease, referred to as self-management. On a daily basis, patients have to take responsibility for a large number of behavioural choices and activities to manage their condition, predominantly outside the health-care setting (Jarvis, Skinner, Carey et al., 2009). The choices that patients make during daily life have a greater impact on health outcomes than those made by health professionals. Once patients are outside the healthcare setting, they themselves are in control; they decide whether or not to 
implement recommendations in their daily life (Funnell \& Anderson, 2004). Patients' choices affect glucose control in the short term, with the risk of hypoor hyperglycaemia, and on a long term basis, the choices affect the risk of complications and the prognosis for life.

Self-management in diabetes goes beyond medication adherence. Selfmanagement consists of behavioural, cognitive, and emotional responses to a constantly changing state of one's condition (Barlow, Wright, Sheasby et al., 2002). On a behavioural level, specific meal planning and sufficient physical activity are highly important self-management behaviours and also smoking cessation is strongly advocated. Additionally, patients have to take their medication as described and sometimes, in case of insulin treatment, patients have to adapt their medication dose or time schedule to specific daily circumstances (e.g. when someone spontaneously decides to work in the garden). What is most difficult is finding the optimal balance between food intake, physical activity and medication effects (Bonnet, Gagnayre, \& d'Ivernois, 2001). Furthermore, patients have to monitor their condition. On the one hand, there are medical examinations by health care professionals such as blood pressure and weight measurements, laboratory measurements and eye and feet examinations. On the other hand, patients have to check their feet and many patients have to monitor their blood glucose levels regularly. Also, symptom management requires patients to be constantly alert to symptoms related to diabetes (e.g. hypoglycaemia), to respond adequately, and to be aware of signs of complications. Additionally, management of psychological consequences is important, e.g. disease acceptance, dealing with depressive feelings and stress management (Snoek \& Hogenelst, 2008). Disease acceptance is important but difficult; when people are diagnosed with diabetes, they are confronted with a chronic illness which most often cannot be cured and lifelong disease management is required. Moreover, patients should deal effectively with their environment. Getting social support and finding optimal ways to communicate with health care professionals is also part of selfmanagement.

Diabetes self-management is complex and emotionally challenging. Patients should be able to solve daily problems, should cope adequately with their condition, and should be able to achieve treatment goals. To do all this, patients need to understand diabetes and its management. Having knowledge is not enough; patients have to apply their knowledge in daily situations such as balancing their diet. What may be equally important is that patients need the confidence to take their role as decision maker and to take their responsibilities for day-to-day diabetes care. The complexity of good self-management requires 
motivated and well educated patients and to support patients optimally, high quality care is needed in which two factors are crucial (Funnell \& Anderson, 2002). First, patients should be prepared to be effective self-managers of their diabetes by providing them with the information and support necessary to make informed decisions. Second, health care system approaches that facilitate ongoing monitoring, support and routine care are needed. Previously, health care systems were merely designed for the treatment of acute health care problems but during the last decades, diabetes care has changed.

\section{DIABETES CARE}

Changes in the view on diabetes and chronic diseases with more emphasis on self-management require a different care model than the traditional routines used for acute illnesses. In this paragraph changes in diabetes care are described as well as the self-management difficulties patients and professionals in diabetes care experience.

\section{Changes in diabetes care}

The changes in diabetes care are twofold; on the one hand, the organization of diabetes care has changed and on the other hand, the content of care changed.

Previously, patients with type 2 diabetes received care from a general practitioner and/or an internist. Due to the ongoing growth in the prevalence of diabetes and the specific type of care that diabetes requires, as in other Western countries, the organization of Dutch diabetes care had to change to be able to provide high quality, cost-effective and easily accessible care.

In response, during the last decade, vertical substitution of care took place, which refers to the transfer of tasks between care-providers with different levels of expertise (Vrijhoef, Diederiks, Spreeuwenberg et al., 2001). Nowadays, care for diabetes type 2 patients is mainly provided in primary care and in many general practices diabetes care is increasingly delegated to socalled 'practice nurses'. These trained practice nurses specialize in chronic care, such as diabetes, and are supervised by the general practitioner. The majority of patients are seen quarterly by a practice nurse and annually by their general practitioner (Diabetesvereniging Nederland, 2008; Nederlandse Diabetes Federatie, 2007). The nurse consultations enable regular follow-up visits and the nurses' main tasks are to monitor the disease and provide education and lifestyle counselling. Training programmes for nurses are more 
focused on the behavioural and preventive aspects of health care than programmes for physicians. Their educational background and specific tasks in daily practice make these nurses the most suitable health care professionals to support patients' self-management. Besides nurses and general practitioners, health care professionals involved in diabetes care may include dieticians, ophthalmologists, and sometimes internists and cardiologists. In contrast, patients with type 1 diabetes and patients with complicated type 2 diabetes needing more complex care are usually treated by internists and specialized diabetic nurses. To provide good quality care, diabetes care standards and guidelines have emerged which describe when what should be done (Nederlandse Diabetes Federatie, 2007).

Not only the organization of diabetes care has changed, also the content has changed during the last two to three decades (Funnell \& Anderson, 2003; Menon, 2002). Funnell \& Anderson (2004) provided a clear description of the shift within diabetes care and education. Previously, care and education were based on a medical model in which health care providers were seen as the authority and patients were expected to comply with the recommendations of their provider. This approach appeared not to be effective in diabetes care (Clement, 1995; Street, Piziak, Carpentier et al., 1993). A shift came towards a more empowering approach, doing justice to the responsibility and control of patients themselves in daily care. Empowerment in the health context is characterized by "perceptions of control regarding one's own health and health care; perceptions of competence regarding one's ability to maintain good health and manage interactions with the health care system; and internalization of health ideas and goals at the individual and societal level" (p.34) (Menon, 2002). The idea of empowerment and of self-management is based on the premise that people have the capacity to make choices and are responsible for the consequences of their choices (Feste \& Anderson, 1995). It is not the health professional but the patient that needs to be in control in order to perform adequate self-management on a daily basis. However, in the situation of diabetes care, patients are (partly) dependent on the expertise of their health care professional. As described in the chronic care model (Wagner, Austin, Davis et al., 2001; Wagner, Bennett, Austin et al., 2005), patient outcomes are a result of productive interactions between informed, activated patients on the one hand and a prepared, proactive team of health care professionals on the other. Nowadays, care is increasingly seen as a collaboration between equals in which the professionals are seen as experts about diabetes and its treatment and patients are seen as experts on their lives and what will work for them (Funnell $\&$ Anderson, 2004). This approach is also adopted by the Dutch Diabetes 
Federation which characterizes the patient as central co-ordinator of his / her own health care plan so that care is more tailored to the individual (Nederlandse Diabetes Federatie, 2007).

\section{Diabetes care and self-management}

Despite organizational and content changes in diabetes care, patients experience difficulties in maintaining adequate self-management resulting in suboptimal health outcomes with a decreased length and quality of life (Funnell \& Anderson, 2004; Resnick, Foster, Bardsley et al., 2006). A study among patients with type 2 diabetes described by Harris et al. showed that despite high health care access and utilization, glycaemic control was poor, many patients were obese and much of the hypertension and dyslipidemia was not controlled (Harris, 2000). Harris gave multiple reasons for this discordance including patient self-care practices and physician medical care practices. An American survey among adults with type 2 diabetes $(n=1480)$ revealed that the majority of these individuals did not meet the guidelines for physical activity and diet, and accordingly were overweight (Nelson, Reiber, \& Boyko, 2002). This indicates patients' difficulties with two highly relevant self-management behaviours (Boulé, Haddad, Kenny et al., 2001; Hu, Manson, Stampfer et al., 2001). These data probably also apply for the Netherlands, e.g. in the region Maastricht / Heuvelland the mean BMI of diabetic patients treated by general practitioners was $29.8 \mathrm{~kg} / \mathrm{m}^{2}$ and only $40 \%$ of the population met a target BMI between $19-27 \mathrm{~kg} / \mathrm{m}^{2}$ (Stichting Regionale Huisartsenzorg Heuvelland, 2010).

Diabetes management is not only a struggle for patients; it is also a challenge for health care professionals. According to primary care professionals, diabetes is harder to treat than other chronic conditions, e.g. hypertension and angina pectoris (Larme \& Pugh, 1998). This is partly due to the fluctuations in glycaemic control and complex medical treatment. An additional complicating factor in motivating patients to change their behaviour is that inappropriate behaviour does not lead to immediate worsening of the disease (Matsuoka, Kawata, Ikeda et al., 1994). Another factor that makes it difficult is that successful management relies to a greater extent on lifestyle change, which is outside the control of the professional. Moreover, once diabetes complications have developed they can impair the patient's lifestyle change abilities. The professional's perceived urgency to control diabetes sometimes contrasts sharply with patients' experiences and perceptions (Larme \& Pugh, 1998). Also, in another study among primary care physicians, patients' non-adherence was perceived as the major barrier to diabetic care 
(Drass, Kell, Osborn et al., 1998). Despite the attempts to encourage and persuade patients to perform self-care tasks, health care professionals are often discouraged when patients do not follow the well-intended advices given (Funnell \& Anderson, 2004). Type 2 diabetes is often a result of a longstanding unhealthy lifestyle in patients with a genetic predisposition. Not only these factors, but also influences of the community and social context referring to factors such as work, family, neighbourhood, community support and (dis)encouragement of behaviours consistent with diabetes regimen recommendations (Glasgow, 1995) make lifestyle change counselling intricate.

In summary, the difficulties health care professionals experience in diabetes care, the suboptimal self-management by patients, the required empowerment of patients, and the increasing prevalence of diabetes type 2 strengthen the need to develop interventions which can contribute to knowledge about the best way to support patients towards effective self-management.

\section{AIMS OF THE CURRENT RESEARCH PROJECT}

The current research project aimed to develop a new evidence based diabetes education and counselling strategy targeted at patients and health care professionals, to evaluate the strategy (in terms of effect and process), to give an overview of recently developed self-management interventions and to give implications for future initiatives in diabetes care and for future research. The project described in this dissertation was targeted at patients with type 2 diabetes and practice nurses.

\section{INGREDIENTS FOR A NEW DIABETES EDUCATION AND COUNSELLING STRATEGY}

The growing interest for the empowerment approach, especially in chronic care, is also seen in the focus of self-management interventions. For example, increased emphasis has been placed on a collaboration between patients and health care professionals and patient centered care, and at the same time, the emphasis on compliance and didactic learning has decreased (Funnell \& Anderson, 2003).

What is known from previous studies about improving patients' selfmanagement, is that active patient participation (Bosworth, Oddone, \& Weinberger, 2006; Funnell \& Anderson, 2002; Rollnick, Mason, \& Butler, 
1999; Van Dam, Van der Horst, Van den Borne et al., 2003; Williams, McGregor, Zeldman et al., 2005), informed shared decision making (Funnell \& Anderson, 2002; Van Dam et al., 2003), and motivating patients for behavioural change by setting concrete, realistic goals (Van Dam et al., 2003; Wens, Vermeire, Hearnshaw et al., 2008) are beneficial. Active patient participation and shared decision making are closely related. In fact, shared decision making is a method to achieve an active partnership between patient and clinician. On the spectrum of patient-professional interaction, shared decision making is the middle ground between paternalism (professional decides what is thought best) and informed choice (patient is provided with information without further guidance) (Elwyn, 2001). It is the process where health care decisions are based on mutual agreement between patient and professional (Frosch, Robert, \& Kaplan, 1999). Another vital component for counselling strategies is education (Clement, 1995). Although knowledge alone is not enough to improve self-management, sufficient understanding of diabetes is necessary to enable problem solving, patient participation and informed, shared decision making (Clement, 1995; Van Dam et al., 2003; Wens et al., 2008). For sustained effects of education, one-time educational programmes rarely succeed (Snoek \& Skinner, 2005). Patients need ongoing support and education, addressing personal needs which vary over time as their condition and personal circumstances change.

The empowerment approach requires health care professionals to adapt their counselling style to the specific features of chronic care. A promising behaviour change counselling strategy is 'Motivational Interviewing' (MI) which is defined as a 'directive, client-centred counselling style for eliciting behaviour change by helping clients to explore and resolve ambivalence' (Miller \& Rollnick, 2002). MI advocates active patient participation and seems a valuable tool to achieve shared decision making. In contrast to traditional, more paternalistic, counselling styles, MI gives the knowledge and experiences of patients a central role in finding the best behaviour change strategies. The motivation to change should originate from the patient instead of being imposed by the health care professional. The four basic principles of MI are: expressing empathy, developing discrepancy between deeply-held values and day-to-day behaviour, rolling with resistance and supporting self-efficacy (Miller \& Rollnick, 2002; Rollnick et al., 1999). Although MI was originally developed in the addiction field, it is increasingly being advocated in other health areas such as diet, exercise, and diabetes (Hettema, Steele, \& Miller, 2005; Miller \& Rose, 2009; Resnicow, Dilorio, Soet et al., 2002). 
Interventions with an MI-based counselling approach might be valuable in improving patients' self-management. However, in earlier research it was shown that interventions focused on health care professionals are less effective than interventions targeting directly at patients (Van Dam et al., 2003). This may argue for patient focused interventions. Patients should be enabled and provided with tools to participate as an equal partner in the health care process. Nevertheless, active patient participation occurs during consultations with professionals. Shared decision making needs at least two parties that both share information and take steps to build consensus about the preferred treatment (Charles, Gafni, \& Whelan, 1997). Therefore, we belief that interventions should be aimed at both, patients and professionals.

Based on the knowledge obtained from previous studies, we developed an intervention consisting of two components;

1. A web-based diabetes education programme to increase patients understanding of type 2 diabetes, to provide tools to support adequate selfmanagement, and to stimulate active patient participation.

2. A Motivational Interviewing based counselling training for practice nurses to implement a new counselling strategy during regular primary diabetes care consultations.

\section{OUTLINE OF THIS DISSERTATION}

In Chapter 2 a description of the development process of the web-based education programme (www.diep.info) is given, which was based on a framework for decision making during programme development: Intervention Mapping. The chapter reports on the needs assessment we conducted, the formulated objectives, the theory-based methods and practical strategies, the programme itself, and on the adoption, implementation and evaluation plan.

In Chapter 3 the effect and user evaluation of the education programme are described. For the effect evaluation a randomized controlled trial (RCT) with a pre-test and post-test design was carried out using a knowledge questionnaire. The user evaluation consisted of an online questionnaire and one-on-one interviews to examine the perceived quality, the use of provided tools, and the use of our programme as a supportive aid in education.

Chapter 4 reports on the content and on the effect evaluation of the Motivational Interviewing (MI) - based counselling training for nurses in diabetes care. By means of an RCT with a pre-test and post-test design, effects 
on clinical, behavioural and process outcomes were assessed among diabetes type 2 patients.

Closely related to the content of chapter 4, in Chapter 5 the effect of the training for nurses on the level of use of the MI-based method in diabetes consultations is shown, evaluated by means of audio-taped consultations of trained versus untrained nurses. Furthermore, an exploration of factors influencing the use of the MI method is given, based on telephone interviews.

Diabetes care has changed over the past years, with much more attention being paid to self-management. In Chapter 6 a review of the most recently (2000-2010) published reports on type 2 diabetes self-management interventions is given, to determine the educational focus and the effectiveness of these interventions.

In the final chapter, Chapter 7, an overview of the research described in this dissertation is given followed by the general discussion. Our project is discussed from a practical as well as a methodological perspective and recommendations for future research and practical implications are given. 


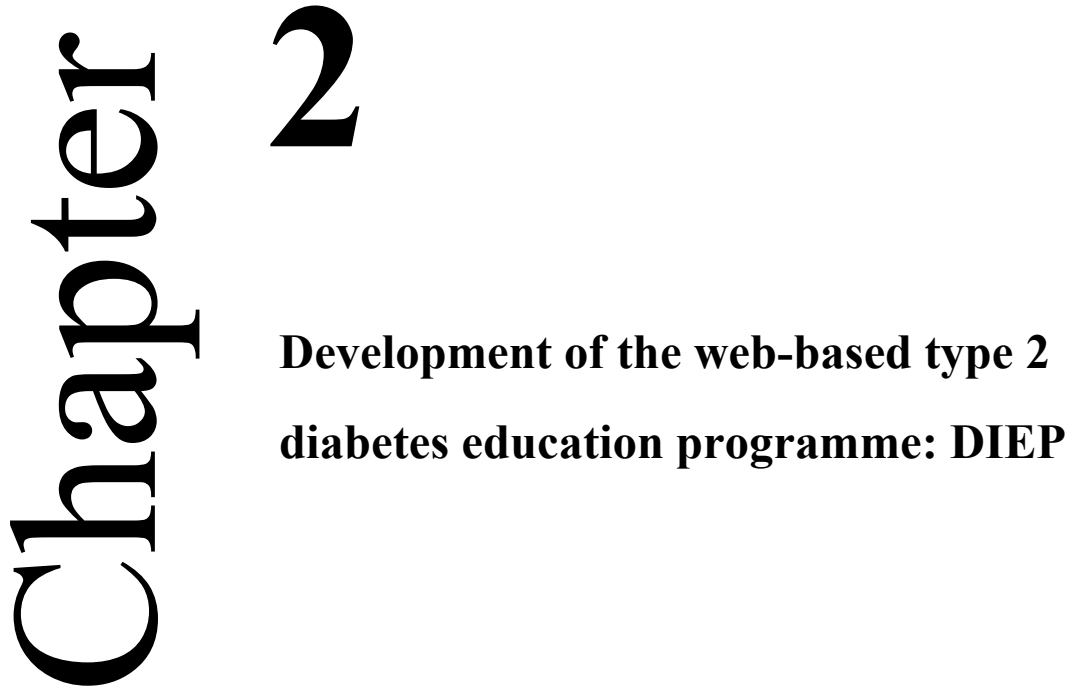

Published as: Heinrich, E., Schaper, N.C., \& De Vries, N.K. (2009). Development of the web-based type 2 diabetes education programme: DIEP. European Diabetes Nursing, 6, 51-56. 


\begin{abstract}
Education is an essential part of diabetes care. However, in the Netherlands, no education programme was available for everyone at any time and adaptive to user's specific needs. The aim of this article is to describe the structured development and final content of a type 2 diabetes web-based education programme.

A web-based education programme - the Diabetes Interactive Education Programme (DIEP) - was developed using intervention mapping and involved collaboration between programme planners, Dutch diabetes organizations and potential users (patients and health care professionals). DIEP incorporates information, multimedia and tools to support self-reflection, goal setting, problem solving and active patient participation.

www.diep.info consists of seven chapters with basic and additional information, a dictionary, self-management checklists and a workbook for goal setting and preparation for consultations. The information included is mostly spoken text supported by headlines, images, video and patient experiences. Adoption, implementation and evaluation plans have been made. Outcomes of the process and effect evaluation will be reported in the future.

DIEP is a unique education programme, based on theory and planned development, which is supported by diabetes organizations. By using multimedia and incorporating different functionalities, DIEP attempts to meet the current practice requirements. DIEP aims to meet the needs of multiple, specific patient groups in the future, and has already been adapted for use in different countries (e.g. Belgium).
\end{abstract}




\section{INTRODUCTION}

Education is an essential component of the care of patients with type 2 diabetes, and understanding the condition is a prerequisite for adequate selfmanagement (Clement, 1995; Funnell \& Anderson, 2002). Patient education is described as 'the process of providing the person with diabetes with the knowledge and skills needed to perform self-care, manage crises, and make lifestyle changes required to successfully manage this disease' (Clement, 1995).

The importance of education is acknowledged worldwide. Although national and international standards for diabetes education have been set (Belton, 2003; Nederlandse Diabetes Federatie, 2005), the content of education is very variable. Different health care professionals (HCPs) use different materials, distributed by different organisations and companies. Materials are mostly written leaflets that address specific topics (e.g. hypoglycaemia). However, to our knowledge, no programme within the Netherlands could be used by all HCPs and patients. Nothing could provide information and tools for self-management at individuals' convenience, adapted to their specific needs. Therefore, we aimed to develop a new education programme for patients recently diagnosed with type 2 diabetes. The programme is based on national guidelines, and was developed in close cooperation with all partners in diabetes care.

During intervention development, careful planning and the use of theorybased strategies increases the probability of effectiveness, programme adoption and implementation (Kok, Van den Borne, \& Mullen, 1997). Intervention mapping (IM) provides programme developers with a framework for decision making (Bartholomew, Parcel, Kok et al., 2000). Accordingly, we conducted a needs assessment, formulated objectives, selected theory-based methods and practical strategies, developed the programme, formulated an adoption and implementation plan, and generated an evaluation plan. Furthermore, the developers of IM explicitly emphasise the importance of stakeholder participation during all planning phases (Bartholomew et al., 2000). This approach enables collaboratively developed user-relevant programmes, and facilitates programme adoption and implementation.

Inspired by an education programme developed at the University of Warwick, and based on IM, the Diabetes Interactive Education Programme (DIEP) was developed in the Netherlands to offer a nationwide, uniform, education programme for the large and rapidly growing group of patients with 
type 2 diabetes. This article describes the structured development and content of DIEP.

The development process was managed by the following: a planning group (three employees of Maastricht University for developmental guidance and research, three employees of the University Hospital Maastricht for programme content and research advice, and one project manager for logistical and financial support); a linkage group (representatives of relevant Dutch diabetes organisations who supported the planning group by intensive communication through meetings, e-mail and telephone contact); and a pilot group of patients with type 2 diabetes. The tasks of these groups are described below.

\section{Needs assessment (NA)}

A needs assessment (NA) was undertaken to analyse the target group and its problems, and to understand 'what is' compared with 'what is more desirable' (Bartholomew et al., 2000). We achieved this by reviewing literature and conducting focus-group interviews (FGIs) among potential programme users.

Our literature review confirmed that patients experience difficulties with self-management (Clement, 1995; Vermeire, Wens, Van Royen et al., 2005a). Furthermore, we identified the following factors associated with improving self-management: active patient participation (Funnell \& Anderson, 2002; Rollnick et al., 1999; Van Dam et al., 2003; Williams et al., 2005); informed decision making (Funnell \& Anderson, 2002); motivating patients for behaviour change by setting concrete, realistic goals (Van Dam et al., 2003; Wens et al., 2008); and education (Norris, Engelgau, \& Narayan, 2001; Norris, Lau, Smith et al., 2002). Although knowledge alone is not enough to improve self-management, it is necessary to enable problem solving, patient participation and informed decision making (Clement, 1995; Van Dam et al., 2003; Wens et al., 2008).

Additionally, four FGIs were carried out to examine the target population's experiences and needs (Ferrell, Grant, Funk et al., 1997; Krueger \& Casey, 2000; Van Assema, Mesters, \& Kok, 1992): one involved HCPs $(n=8)$ and three involved patients with type 2 diabetes (total $n=25$ ). No ethical approval was obtained for these interviews, but all participants provided written informed consent. Participants' responses were recorded and fully transcribed. The content analysis was based upon Morgan's qualitative research methods (Morgan, 1988). Data from HCPs and patients were analysed separately.

One of the most difficult tasks reported by HCPs was to motivate patients for behaviour change. HCPs commented that optimal treatment outcomes are 
often impossible to achieve. Therefore, treatment goals should be personalised and related to the possibilities for each individual. Furthermore, HCPs argued that many patients have insufficient understanding of their disease in order to participate as equal conversation partners, to share responsibility and to formulate goals. It was mentioned that patients' free choice is often the last option, if counselling based upon advice by HCPs does not work.

Concerning education, a need for a univocal education tool adapted to the population became apparent. HCPs felt that formulating achievable goals with the patient would be beneficial.

Patient FGIs revealed that patients felt little need for further education: they reported sufficient levels of information. However, patients kept asking the moderator and assistant questions, such as 'How do you get diabetes?', or 'Why are carbohydrates so important?'. Furthermore, patients said that they received large quantities of very general information, which was not always directly applicable to their personal situation. Often, the reasons for making recommendations remained unexplained, and patients felt a need to look at the information again at home. Most patients said that they just listen to recommendations made by the HCPs; none of the patients reported that they had ever been asked what they wanted. According to patients, goal setting was undertaken, but only in general terms, e.g. 'Lose some pounds'.

A comparison between the outcomes of our literature review and the FGIs identified possible gaps in current practice, thereby revealing the needs of HCPs and patients with type 2 diabetes. When the outcomes were presented, all members of the linkage group subscribed to the findings, supported by their own experiences.

\section{Objectives}

Based on our NA, the following goals for our education programme were set:

- To increase understanding of type 2 diabetes.

- To support communication between HCPs and patients, and stimulate active patient participation.

- To provide tools to support adequate self-management. 


\section{METHODS AND STRATEGIES}

Based on the previous steps, the following strategies were considered:

- To increase understanding of type 2 diabetes and to improve selfmanagement, the website should contain useful information.

- To prevent an overload of information, patients should not be exposed directly to all content.

- To increase the probability of a better understanding of the information provided, multimedia should be used (Jeste, Dunn, Folsom et al., 2008).

- To stimulate self-reflection and understanding of type 2 diabetes and selfmanagement, questions about the patient's specific situation should be included.

- To further support self-management and problem solving, goal-setting forms and checklists that give advice for specific situations should be incorporated into the website.

- To support active patient participation and communication between HCPs and patients, tools that enable patients to prepare for consultations should be included so that patients can participate actively in agenda setting.

A website was chosen as the delivery method because it is easy to update when new diabetes care guidelines are produced, and it is available for every HCP and patient at every moment. Furthermore, the number of people using the internet to seek health-related information is growing (Kaiser Family Foundation, 2005). Of internet users in Europe, 71\% are searching for health information (Andreassen, Bujnowska Fedak, Chronaki et al., 2007). In the Netherlands, the penetration of the internet exceeds $90 \%$ (Internet World Stats, 2008).

\section{RESULTS}

\section{Programme development}

DIEP gives an overview of type 2 diabetes in seven chapters covering: background information about the disease; hyperglycaemia, diabetes and lifestyle; disease management; hypoglycaemia; blood glucose monitoring and living with diabetes. Each chapter provides basic information (a simple description of a topic, e.g. where insulin is produced) and additional information (more detailed and with the use of medical terms, e.g. the 
relationship between insulin and blood glucose). Each chapter closes with questions for the patient, such as 'What is your strategy to prevent hypoglycaemia?'.

The information is mostly presented in spoken language, supported by headlines, images, video (e.g. a demonstration of insulin injection) and patient experiences. The navigation options and page design are adapted to the target population. Each chapter has a workbook to record information that has already been discussed with a patient (Figure 2.1), and a goal-setting sheet that enables plans for improved self-management to be discussed by the HCP and patient (Figure 2.2). In addition, the patient can prepare for HCP consultations, by writing down questions or remarks. Furthermore, the programme contains a dictionary of relevant concepts and links to relevant paragraphs. Additionally, three patient information leaflets about foot care, travelling and sickness can be printed out and used as checklists.

The planning group developed the final programme, working in association with a multimedia company that specialises in education. However, every suggestion for content, functionality and characteristics was discussed with the linkage group until an agreement was achieved. After developing three chapters, a test version was shown to the pilot group to gain feedback from potential users. This collaborative effort led to a web-based education programme, www.diep.info, which is endorsed by all members of the linkage group.

\section{Adoption and implementation plan}

Among patients, DIEP is promoted by advertisements on diabetes websites and in diabetes magazines, and by leaflets available from general practices. However, HCPs are the main channel for promotion. We hypothesised that implementation will succeed if HCPs use DIEP as their education tool during consultations, and if they recommend that patients use DIEP at home. Therefore, a prerequisite for the use of DIEP by patients is to embed it into standard care.

Among HCPs, DIEP is promoted through publications in specialist journals, presentations and demonstrations at congresses, and during personal visits by representatives of the diabetes care industry. In addition, to stimulate optimal use of DIEP, training for HCPs has been developed (diep@work).

The linkage group was highly important for realising this plan. By involving representatives of relevant Dutch diabetes organisations, we created support and thereby gained opportunities to promote DIEP. 


\begin{tabular}{|l|l|}
\hline Diabetes background \\
\hline \multicolumn{2}{|l|}{ Date: } \\
\hline $\begin{array}{l}\text { What is diabetes? } \\
\text { How do I know I have diabetes? }\end{array}$ & \\
\hline [more specific info] & \\
\hline $\begin{array}{l}\text { How does insulin work? } \\
\text { Diabetes, a growing problem } \\
\text { Heredity factors }\end{array}$ & \\
\hline Which of the following questions are still difficult for you, after reading this chapter? \\
\hline $\begin{array}{l}\text { What caused your diabetes? } \\
\text { Which risk factors are relevant to you? } \\
\text { How did you follow up the advice given with regard to weight loss and/or } \\
\text { increasing exercise? }\end{array}$ & \multicolumn{2}{|l|}{} \\
\hline Remaining questions or things you want to discuss: & \\
\hline Discussed with:
\end{tabular}

Figure 2.1 Example of a workbook page for patients to complete

\section{Evaluation plan}

We developed an online questionnaire to study to what extent DIEP is effective in improving knowledge about type 2 diabetes in a randomised controlled trial with pre-test/post-test design. The intervention consists of two weeks' access to DIEP for the experimental group; the control group is only permitted access after the post-test.

A process evaluation is carried out using an online questionnaire that assesses the quality of DIEP (including satisfaction with content and userfriendliness), the functionality and the use of the website as a supportive educational tool. Additionally, semi-structured one-to-one interviews are held to obtain more detailed user information, addressing quality aspects of online education materials and the opportunities and aims of DIEP. Furthermore, we observe DIEP users while working with the programme.

The evaluation outcomes are beyond the scope of this article but will be reported in the future. 


\begin{tabular}{|c|c|}
\hline $\begin{array}{l}\text { Date of appointment: } \\
\text { Current status }\end{array}$ & Goals \\
\hline $\begin{array}{l}\text { Weight: } \\
\text { Length: } \\
\text { Body mass index: } \\
\text { Waist circumference: }\end{array}$ & $\begin{array}{l}\text { Weight reduction? } \\
\text { Yes / No If yes, how much a week? } \\
\text { How? } \\
\text { More physical activity / eat less / both }\end{array}$ \\
\hline $\begin{array}{l}\text { Do you smoke? } \\
\text { Yes / No } \\
\text { If yes, what? } \\
\text { How much a day? }\end{array}$ & $\begin{array}{l}\text { Do you want to quit smoking? } \\
\text { Yes / No } \\
\text { If yes, how? }\end{array}$ \\
\hline $\begin{array}{l}\text { What physical activity do you perform? } \\
\text { a. Strenuous physical work } \\
\text { b. Brisk walking } \\
\text { c. Cycling } \\
\text { d. Something else: } \\
\text { Does your daily physical activity last at least half } \\
\text { an hour a day? } \\
\text { Yes / No }\end{array}$ & $\begin{array}{l}\text { More physical activity? } \\
\text { Yes / No } \\
\text { If yes, how? } \\
\text { How much extra time per day or per week? }\end{array}$ \\
\hline $\begin{array}{l}\text { What do you eat on an average day? } \\
\text { Breakfast: } \\
\text { Lunch: } \\
\text { Dinner: } \\
\text { Snacks: } \\
\text { How many? } \\
\text { What? }\end{array}$ & $\begin{array}{l}\text { Eat less or differently? } \\
\text { Yes / No } \\
\text { If yes, what do you want to change? } \\
\text { Breakfast: } \\
\text { Lunch: } \\
\text { Dinner: } \\
\text { Snacks: }\end{array}$ \\
\hline $\begin{array}{l}\text { What do you drink on an average day? } \\
\text { Soft drinks: } \\
\text { Alcohol: }\end{array}$ & $\begin{array}{l}\text { Drink less? } \\
\text { Yes / No } \\
\text { If yes, what do you want to change? } \\
\text { Soft drinks: } \\
\text { Alcohol: }\end{array}$ \\
\hline & \\
\hline
\end{tabular}

Figure 2.2 Goal-setting sheet, for health care professionals to use during patient consultations 


\section{DISCUSSION}

DIEP is a unique education programme, created using theoretical models and planned development strategies, and supported by diabetes organisations in the Netherlands. While other web-based education programmes merely offer text (e.g. www.diabetes.org or www.dvn.nl), DIEP uses multimedia and different functionalities, thereby attempting to meet the needs of current practice. This programme is available for anyone at any time. In 2006, DIEP received the Dutch quality award for diabetes care. Further implementation of DIEP and future research is needed to examine whether our objectives have been achieved.

A shortcoming of our developmental process may be that we adapted the steps of IM to suit what was feasible within our time and budget. However, we followed the main IM steps and, during the whole developmental process, collaboration between programme planners, implementers and users was ensured.

To guarantee ongoing programme development and improvement we have established the DIEP foundation. Currently, DIEP is targeted at patients who have recently been diagnosed with type 2 diabetes. Future developments may extend the programme to different target groups or adapt the content to users' characteristics (e.g. age or knowledge). Furthermore, DIEP can be adopted by other countries. In fact, Belgium has already adapted the programme. Such developments enable DIEP to become an international programme that is usable by different HCPs and different patient groups.

\section{ACKNOWLEDGEMENTS}

Thanks to all health care workers and patients who were involved in the development of DIEP, in particular: J. Brouwer, H. van Dam†, E. DenisThissen, R. van Doorn, M. Mohr, A. Ovink, C. Spreeuwenberg, J. de Waal and Y. Wils. Furthermore, we would like to thank the Dr. Paul Janssen foundation, of Johnson \& Johnson healthconnaction, for their financial support and in particular J. de Waal of LifeScan, for his logistic support. 


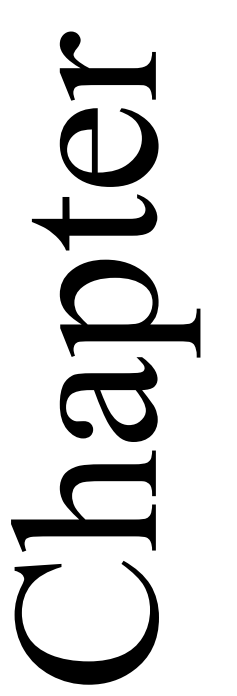

Evaluation of the web-based Diabetes

Interactive Education Programme (DIEP)

for patients with type 2 diabetes

An adapted version is accepted as: Heinrich, E., De Nooijer, J., Schaper, N.C.,

Schoonus-Spit, M.H.G., Janssen, M.A.J., \& De Vries, N.K. Evaluation of the web-based

Diabetes Interactive Education Programme (DIEP) for patients with type 2 diabetes.

Patient Education and Counseling. 


\begin{abstract}
The objective of the present study was to evaluate a web-based type 2 diabetes selfmanagement education programme aimed at improving knowledge, stimulating active patient participation and providing supportive self-management tools.

Two studies were performed; (1) for the effect evaluation an RCT with a pre-test and post-test design ( $\mathrm{n}=99$ ) was carried out using a knowledge questionnaire; (2) the user evaluation consisted of an online questionnaire $(n=564)$ and one-on-one interviews $(n=11)$ to examine the perceived quality, the use of functionalities and the use of our programme as a supportive tool in education.

The effect evaluation showed a significant intervention effect $(p<0.01)$ on knowledge. The user evaluation showed high satisfaction with the content, credibility and user-friendliness of the programme. However, functionalities and self-management tools were used by less than half of the participants.

The programme can improve knowledge but it is not fully used as intended. A more optimal use of the programme is necessary for higher efficacy. The use of mostly spoken text instead of written text was highly appreciated and could be used more often for educational websites. Furthermore, health care professionals need support in implementing new educational programmes during consultations.
\end{abstract}




\section{INTRODUCTION}

Self-management is an essential component of the management of chronic illnesses, as patients are in control of and responsible for the daily management of their disease (Funnell \& Anderson, 2004). Adequate self-management is complex, but it can prevent or delay the onset of complications (Adler, Stratton, Neil et al., 2000; Barlow et al., 2002; Norris et al., 2002; UK Prospective Diabetes Study Group, 1998) and thereby it can limit the impact of chronic illness on patient and societal level.

Diabetes type 2 (DM2) is a chronic condition with a fast increasing prevalence. The WHO estimated a rise of the total number of people with DM from 171 million in 2000 to 366 million in 2030 (Wild, Roglic, Green et al., 2004), of which approximately $90 \%$ have DM2. This increase will place an extra load on the diabetes care system. Earlier studies have shown that patients with chronic conditions experience difficulties with self-management (Clement, 1995; Jerant, Von Friederichs Fitzwater, \& Moore, 2005; Vermeire, Wens, Van Royen et al., 2005b), but the time health care professionals (HCPs) can devote to education per patient decreases. Therefore, to limit the consequences of the 'diabetes epidemic', supportive tools for adequate selfmanagement are urgently needed.

From earlier research, prerequisites for better self-management are known. First of all, patients should have enough knowledge to make informed decisions during daily living with DM (Clement, 1995; Funnell \& Anderson, 2004; Funnell \& Anderson, 2002) and to be able to equally collaborate with their health care professional (HCP). Furthermore, active patient participation during consultations is beneficial (Williams et al., 2005; Williams, McGregor, Zeldman et al., 2004). Patients who are involved in goal setting and who have clear goals are more likely to be successful in achieving them (Clark, Hampson, Avery et al., 2004a; Heisler, Bouknight, Hayward et al., 2002). Therefore, shared goal setting can be helpful in realizing self-management goals.

Although these different valuable strategies are known, within the Netherlands education materials in DM2 care primarily consist of written materials, mostly focused on didactic teaching. Also, different organisations and companies distribute different education materials through HCPs. To our knowledge, within our country there was no nation-wide programme that could be used by different HCPs and also by DM2 patients, providing the information and tools for self-management at their convenience and adaptive to specific needs. 
To support patient education and thereby self-management, we developed a web-based education programme (Diabetes Interactive Education Programme; DIEP) for DM2 patients. The number of people using Internet for information about health is growing (Kaiser Family Foundation, 2005) and websites are available for anyone with Internet at any moment. Critics warn for the variable quality of information as the credibility and is often unknown or difficult to evaluate (Cline \& Haynes, 2001). DIEP is developed in close cooperation with potential users and is based on national guidelines for diabetes care. Thereby, it provides users with credible information and tools for self-management. The programme aims to (a) increase understanding of DM2; (b) stimulate active patient participation; (c) provide tools to support adequate self-management. An article about the programme and the structured developmental process that was based on a theoretical framework for intervention planning (intervention mapping) (Bartholomew et al., 2000), is described elsewhere (Heinrich, Schaper, \& De Vries, 2009). A short description of DIEP can be found below.

To evaluate DIEP, two studies were performed. In the first, an effect evaluation, we determined if an internet-based education programme (DIEP) has the potential to contribute to self-management by increasing knowledge. Furthermore, the efficacy depends on the use of the programme and its functionalities by HCPs and patients, and on the match with the needs and preferences of the HCPs and patients. Therefore, a user evaluation among patients and HCPs was carried out in a second study to assess the perceived quality of DIEP, the use of functionalities and the use of DIEP as a supportive tool in education.

This article describes these two studies which were performed in different samples. Results from these studies can be extrapolated to tools for other chronic conditions.

\section{www.DIEP.info}

DIEP gives an overview of DM2 in seven chapters, see Table 3.1. Each chapter provides basic information (e.g. where insulin is produced) and additional information (e.g. relation between insulin and blood glucose). Each chapter closes with questions to the patient, e.g. 'What is your strategy to prevent hypoglycaemia?'.

The programme uses multimedia; the information is mostly presented in spoken language, supported by headlines, images, video and patient experiences. All spoken language can be printed out as written text, and the sound can be switched off. In a workbook per chapter one can register which 
information is already consumed and self-management plans can be described. Also, questions can be written down to discuss with the HCP. Furthermore, the programme contains a search function with links to relevant paragraphs. Additionally, three leaflets can be printed as checklists, e.g. about foot care.

\section{METHODS}

\section{Study 1: Effect evaluation}

Design

In a randomized controlled trial with a pre-test ( $\mathrm{T} 0)$ and one post-test after two weeks (T1) participants were allocated to the experimental group(A), the control group(B) or the post-test only control group(C). The latter was included to assess possible test effects of completing the pre-test on post-test knowledge scores. Only the experimental group(A) had access to DIEP for two weeks. Both control groups received the intervention after the post-test.

\section{Participants and procedures}

DM2 patients were recruited by an announcement in a free DM magazine, in local door-to-door papers and on two websites. Patients could send an email for more information. After informed consent was obtained, patients were allocated to one of the three groups. Inclusion criteria were: diagnosis of DM2, age 40-70. Patients were excluded from participation if they already used DIEP. The questionnaires were sent by e-mail and participants of the experimental group(A) received a login code for DIEP. The 'number of website visits' and 'total time spent on the website' were registered.

\section{Knowledge questionnaire}

The knowledge questionnaire was newly developed because to our knowledge there was no validated questionnaire that was applicable to the Dutch situation. The questionnaire was based on the content of DIEP, see Table 3.1. It contained 29 multiple choice knowledge items with three options of which only one was correct (values: $1=$ correct, $0=$ incorrect). Knowledge scores were calculated by summing all item scores with a maximum of 29. Theoretical knowledge as well as practical knowledge was measured, see Table 3.2. 
Furthermore, additional information was obtained about sex, age, educational level, time since diagnose and medical treatment.

The reproducibility of our knowledge questionnaire was determined by assessing the agreement (Intraclass Correlation Coefficient; two-way random model with participants and moments of measurement as random factors) and the reliability (Pearson's $r$; test-retest method) using pre-test and post-test scores of the control group(B). Both, $\mathrm{ICC}_{\text {agreement }}$ and Pearson's $r$, were 0.85, $p<0.001$, indicating high reproducibility. Furthermore, an indicator of construct validity was the expected relationship between baseline scores and educational level $(\beta=0.315 ; p=0.002)$ and time since diagnosis $(\beta=0.215 ; p=0.027)$ and not between baseline scores and age or gender (Fitzgerald, Funnell, Hess et al., 1998).

Table 3.1 Knowledge scale topics

\begin{tabular}{lc}
\hline Chapter & Number of items \\
\hline Background information about DM & 5 \\
Hyperglycemia (consequences \& treatment) & 4 \\
Diabetes and lifestyle & 8 \\
Treatment \& management of the disease & 5 \\
Hypoglycemia & 3 \\
Blood glucose monitoring & 2 \\
Living with diabetes (managing special situations) & 2 \\
Total & $\mathbf{2 9}$ \\
\hline
\end{tabular}

Table 3.2 Examples of practical and theoretical knowledge questions

\begin{tabular}{cl}
\hline Theoretical knowledge & Practical knowledge \\
\hline Eating foods lower in fat decreases your risk for: & Imagine you are on a birthday party and you drink two \\
& glasses of red wine. Your blood glucose initially will: \\
& a. increase \\
a. nerve disease & b. decrease \\
b. kidney disease & c. stay the same \\
\hline
\end{tabular}

\section{Data analysis}

Independent-sample $t$-tests and logistic regression analyses were used for attrition analysis. To test whether randomization was successful, Chi-square, one-way ANOVA's and independent-sample $t$-tests were used. An independent-samples $t$-test (between the two control conditions) was used to assess whether there was a test effect of the pre-test on post-test knowledge scores. Multiple linear regression analysis was used to examine the relation between demographic characteristics and baseline knowledge scores. 
Within group analyses on knowledge were done by means of a paired $t$-test. The efficacy of the intervention was further tested with a multiple linear regression analysis. Significance of effects is reported $(p<0.05)$ as well as the magnitude of the effect size (Cohen's $d$ ) (Cohen, 1988).

To determine if a relation exists between total time spent on the website and change in knowledge a Spearman correlation analysis was done because total time spent was not normal distributed.

Participants with an outlying (outside three standard deviations from the mean) 'total time spent on the website' or 'number of website visits' were excluded from the analyses with these variables.

\section{Study 2: User evaluation}

For the user evaluation, an online questionnaire was used. More explanatory information was obtained in eleven semi-structured one-on-one interviews.

\section{Participants and procedures}

All visitors of DIEP were asked to fill out an online questionnaire the moment they wanted to close the programme. Only the data of patients with DM2 were included in the analyses. The questionnaire was available for two years (July 2006 - July 2008).

For the interviews not only DM2 patients but also HPCs (nurse practitioners and diabetic nurses) using DIEP were approached, because optimal use of DIEP as an education tool partly depends on the use by HCPs. The snowball method was used for recruitment; a HCP working with DIEP was asked to name other HCPs working with DIEP and HCPs were asked to recruit patients. Informed consent was obtained prior to the interview.

\section{User evaluation measurements}

The online questionnaire consisted of mostly multiple-choice questions, addressing the perceived quality of the website and the use of functionalities. Table 3.3 gives an overview of number of items per topic and examples of questions. Open ended questions were used for: a) clarification of which information is lacking in the programme; b) suggestions for improvement of the programme.

For the interviews a topic guide was generated based on previous research on quality aspects of websites (Eysenbach, Powell, Kuss et al., 2002; Kerr, Murray, Stevenson et al., 2006; Silberg, Lundberg, \& Musacchio, 1997; Wyatt, 
1997). The main topics were (a) programme content; (b) user-friendliness \& programme features; (c) programme goals, see Table 3.4.

Table 3.3 User evaluation online questionnaire topics

\begin{tabular}{|c|c|c|}
\hline Topic & Number of items & Example \\
\hline Content & 5 & $\begin{array}{l}\text { How satisfied are you with the information you have } \\
\text { found? } \\
\text { a) very satisfied, b) satisfied, c) not satisfied, not } \\
\text { unsatisfied, d) unsatisfied, e) very unsatisfied }\end{array}$ \\
\hline User-friendliness & 4 & $\begin{array}{l}\text { What do you think of the user-friendliness of the website? } \\
\text { a) very good, b) good, c) not good, not bad, d) bad, } \\
\text { e) very bad }\end{array}$ \\
\hline Use of sound & 2 & $\begin{array}{l}\text { If you visit the website, is your sound turned on? } \\
\text { a) yes, b) no, c) no, because my computer has no sound }\end{array}$ \\
\hline Use of functionalities & 4 & $\begin{array}{l}\text { Have you ever looked at the additional information? } \\
\text { a) yes, b) no }\end{array}$ \\
\hline Revisit & 2 & $\begin{array}{l}\text { Are you planning to revisit the website? } \\
\text { a) yes, b) no }\end{array}$ \\
\hline Suggestions & 1 & Do you have suggestions to improve the website? \\
\hline $\begin{array}{l}\text { Background info } \\
\text { (Demographics, duration } \\
\text { of DM2, website visit) }\end{array}$ & 4 & - \\
\hline Total & 22 & \\
\hline
\end{tabular}

Table 3.4 Examples of one-on-one interview questions

\begin{tabular}{ll}
\hline Topic & Example \\
\hline Programme content & What is your opinion about the content of the website? \\
& What information could not be found? \\
User-friendliness & What do you think about the user-friendliness of the website? \\
& What could be improved according to you? \\
Programme goals & What is, according to you, the goal of the website? \\
& Do you think that our goals are achievable? \\
\hline
\end{tabular}

\section{Data analysis}

Concerning the online questionnaire, logistic regression analyses were used to analyze the relationship between the use of functionalities and demographics.

The interviews were all tape-recorded and transcribed verbatim. Analysis was conducted on original transcripts, facilitated by using QSR Nvivo 2.0. 


\section{RESULTS}

\section{Study 1: Results of the effect evaluation}

\section{Response, baseline characteristics and test effect}

The majority ( $87 \%)$ of all people that wanted more information about this study $(n=190)$ gave informed consent $(n=166)$. Thirteen participants were excluded from all analyses because they already had used the website before the study. Nine participants dropped out before the start of the study (no data available), and another nine before the post-test (experimental group(A) $n=7$, control $\operatorname{group}(\mathrm{B}) \mathrm{n}=2$ ).

There were no significant differences in gender, age, BMI, time since diagnosis, educational level or baseline knowledge score between the three groups. Table 3.5 gives a description of the participants' characteristics $(\mathrm{n}=135)$. Participants with a longer time since diagnosis $(\beta=0.215 ; p=0.027)$ or a higher educational level $(\beta=0.315 ; p=0.002)$ had a higher baseline knowledge score than participants with a shorter time since diagnosis or lower educational level. Baseline knowledge did not depend on age or gender.

No test-effects on knowledge scores were found when the post-test knowledge scores of the control group(B) were compared with the post-test only control group $(\mathrm{C})(t=0.01 ; d f=90 ; p=0.99)$.

Table 3.5 Participants characteristics of the effect evaluation and online user evaluation

\begin{tabular}{|c|c|c|c|c|}
\hline \multirow[b]{2}{*}{ Variable: } & \multicolumn{3}{|c|}{ Effect evaluation } & \multirow{2}{*}{$\begin{array}{c}\text { User evaluation } \\
(n=564)\end{array}$} \\
\hline & $\begin{array}{c}\text { Exp group }(A) \\
(n=43)\end{array}$ & $\begin{array}{c}\begin{array}{c}\text { Control group }(B) \\
(n=56)\end{array} \\
\end{array}$ & $\begin{array}{c}\text { Control group }(C) \\
(n=36)\end{array}$ & \\
\hline Sex; \% female & 56 & 48 & 53 & 40 \\
\hline Mean age & $56( \pm 7)$ & $56( \pm 7)$ & $59( \pm 6)$ & $57( \pm 10)$ \\
\hline \multicolumn{5}{|l|}{ Educational level } \\
\hline$\%$ Low & 35 & 43 & 25 & 34 \\
\hline$\%$ Middle & 35 & 30 & 28 & 38 \\
\hline$\%$ High & 30 & 27 & 47 & 24 \\
\hline \multicolumn{5}{|l|}{ Time since diagnosis } \\
\hline$\%<$ years & 19 & 21 & 31 & 44 \\
\hline$\% 2-4$ years & 16 & 27 & 14 & 20 \\
\hline$\% \geq 4$ years & 65 & 52 & 55 & 36 \\
\hline \multicolumn{5}{|l|}{ Treatment } \\
\hline$\%$ None & 7 & 4 & 0 & - \\
\hline$\%$ Oral medication & 56 & 70 & 61 & - \\
\hline$\%$ Insulin & 23 & 9 & 14 & - \\
\hline$\%$ Oral med. \& insulin & 14 & 18 & 25 & - \\
\hline Baseline knowledge score* & $24.56( \pm 2.38)$ & $24.50( \pm 2.84)$ & - & - \\
\hline Post-test knowledge score* & $25.26( \pm 2.11)^{\mathrm{a}}$ & $24.29( \pm 2.96)^{b}$ & $24.28( \pm 3.18)$ & - \\
\hline
\end{tabular}




\section{Intervention effects}

Within the experimental group(A), post-test knowledge scores were significantly higher compared to baseline $(p<0.05 ; \mathrm{ES}=0.40)$. No changes were observed in the control group(B).

Compared to the control group(B), the experimental group(A) had higher knowledge scores on T1 $(\beta=0.18)$ with adjustment for knowledge at T0. This difference did not change after adjustment for age, gender, educational level and time since diagnosis, see Table 3.6. The magnitude of the effect of group on knowledge was medium with an ES of 0.54 (Lipsey, 1990). Furthermore, participants with higher age had a lower post-test knowledge score than younger participants. The group $\times$ age interaction effect was not significant.

Within the experimental group(A), the mean number of website visits was $3.56(\mathrm{SD}=2.66)$ and the total time spent on the website was 58 minutes on average $(\mathrm{SD}=56.08)$. The correlation between total time spent on the website and increase in knowledge was not significant $(p=0.42)$. Furthermore, there was no significant relation between total time spent on the website and baseline knowledge, sex, age, educational level and time since diagnosis.

Table 3.6 Results of linear regression on knowledge at post-test $(n=99)$

\begin{tabular}{lcc}
\hline Variable & $\boldsymbol{\beta}$ & $\boldsymbol{p}$ \\
\hline Group & 0.18 & 0.005 \\
Knowledge T0 & 0.79 & 0.000 \\
Sex & -0.03 & 0.611 \\
Age & -0.13 & 0.047 \\
Educational level & -0.04 & 0.583 \\
Time since diagnosis & 0.04 & 0.505 \\
\hline
\end{tabular}

$R^{2}=0.67$

\section{Study 2: Results of user evaluation}

\section{Participants}

Online questionnaire. Of the 3961 people that were asked to participate, 3122 refused and 275 were excluded from further analysis, because they were not DM2 patients. Finally, 564 patients were included in this study. The average age was 57 years $(\mathrm{SD}=9.96)$, see Table 3.5 for a description of participants' characteristics.

For $84 \%$ of the participants, it was the first time they visited DIEP. Participants gave multiple reasons for visiting the website; $61 \%$ were advised to visit DIEP by their HCP, 35\% wanted information about DM2 without 
having a specific question and $23 \%$ had heard of this website and was curious. Another 10\% had a specific question about DM2. Almost all (99\%) participants had the intention to revisit DIEP.

Interviews. Six HCPs (five women) and five patients (two women) were interviewed. Three HCPs were familiar with DIEP for several months, the other three for longer than one year. Two patients also used DIEP longer than one year, two had used it a couple of months and one patient was only recently introduced to DIEP.

\section{Evaluation of the programme}

Content. The respondents to the questionnaire evaluated the content of DIEP positively; $85 \%$ fully found and $10 \%$ partially found the information they were looking for. Furthermore, 94\% was satisfied or very satisfied with the information, 91\% acquired new knowledge by visiting DIEP and 99\% reported that DIEP was interesting in general. All respondents regarded information of DIEP as credible. Critical notes were that the information on medication and some aspects of diet were difficult to find. Moreover, the amount of information about diet should be increased. The interviews mainly supported these outcomes on satisfaction with content, although some participants would like to see more specific information (e.g. specific diets). Furthermore, all participants estimated the content as credible. Some participants said that they found it reliable because of the involvement of an academic hospital. Almost half of the participants perceived the content as up-to-date; the other participants said this was difficult to assess.

User-friendliness. Almost all (98\%) respondents to the questionnaire described this aspect as good to very good. Furthermore, all respondents agreed that the chapter division is clear and $95 \%$ reported that it was (very) easy to navigate through DIEP. Almost all (96\%) respondents answered that the language use was good. Participants in the interviews explicated that the use of colours in the website contributed to the clarity of the programme, and that the illustrations were useful to understand the information. The distinction between basic information and additional information was highly appreciated and according to HCPs, it protects patients from information overload.

Use of sound. Of the respondents to the questionnaire, 91\% was (very) pleased by the fact that much information is given as spoken rather than written text, although it was suggested to provide all text in writing additionally. Some respondents would like to be able to import their medical information. Data from the interviews showed that most participants (patients as well as HCPs) 
strongly appreciated the spoken text, although HCPs did not know how to switch off the sound while using the programme during their consultations.

Use of functionalities. Of the respondents to the questionnaire, $41 \%$ used the search function, $28 \%$ answered the questions at the end of a chapter, $60 \%$ looked at the additional information and $13 \%$ printed out some spoken text at least once. There was no influence of age or time since diagnosis on the use of these functionalities. However, men were more likely to look at the additional information than women (55\% versus $64 \%$, OR $=1.508 ; 95 \% \mathrm{CI}=1.02-2.20$; $p<0.05$ ), and respondents with a low educational level were less likely to answer questions at the end of a chapter than respondents with a middle $(\mathrm{OR}=1.884 ; 95 \% \mathrm{CI}=1.19-2.99 ; p<0.05)$ or high $(\mathrm{OR}=1.824 ; 95 \% \mathrm{CI}=1.09$ $3.06 ; p<0.05$ ) educational level, $20 \%$ versus $33 \%$ and $31 \%$ respectively. From the participants of the interviews, only two HCPs used the workbook intensively and four others distributed a workbook to their patients without using it during consultations. Only two patients used the workbook to write down their questions to discuss these with their HCP.

Programme goals (only discussed in the interviews). Regarding the intended goals of DIEP (to improve self-management and stimulate patient activation), three HCPs said that DIEP can improve self-management, and after probing another two agreed with that. However, DIEP was primarily seen as an additional information tool in education. Several HCPs argued that DIEP only is not sufficient to achieve better contact and better self-management; individual contact remains crucial. According to patients, the main goal of DIEP is to provide information. And according to three patients the website contributes to problem solving. All patients share the view, when specifically asked, that the website can potentially improve communication and selfmanagement.

\section{DISCUSSION}

We investigated whether an internet based education programme has the potential to contribute to self-management by improving knowledge in DM2 patients. Even though baseline knowledge scores were relatively high and we offered the opportunity to use the programme only for a two week period, we found a positive intervention effect. This was explained by a significant improvement of knowledge scores in the experimental group(A). The relative high baseline scores could be explained by the fact that more than half of all participants had diabetes for more than four years. It is very likely that these 
patients already received DM education as part of their treatment. Our knowledge questionnaire might have been relatively easy for the participants. Although theoretically patients with recently diagnosed diabetes or patients with a low educational level may benefit most from the programme because they had the lowest baseline scores, we did not find a higher increase in knowledge among these patients. Improvements in knowledge were independent of the total time spent on the website, indicating beneficial effects even after viewing only a small part of the total programme.

Earlier, the importance of educating patients for a better understanding of the disease was stressed (Assal, Jacquemet, \& Morel, 1997). Puder and colleagues (2003) emphasized the necessity of educating patients with a chronic condition to enable them to make informed choices concerning health related behaviour and to implement a self-care plan with individual goals (Puder \& Keller, 2003). Therefore, we believe that DIEP can contribute to better self-management by increasing knowledge about DM2. Conversely, the relevance of knowledge as outcome of patient education has been disputed (Cooper, Booth, Fear et al., 2001). Knowledge may not directly lead to better self-management. However, knowledge may be regarded as a prerequisite for self-management; patients with chronic conditions need sufficient understanding of the disease to make day-to-day decisions about their illness (Clement, 1995; Funnell \& Anderson, 2002; Van den Arend, Stolk, Krans et al., 2000; Wens et al., 2008).

In addition to the effect evaluation we assessed the perceived quality of the programme, the use of its functionalities and the use of DIEP as a supportive tool in education in a second study by means of an online questionnaire and one-on-one interviews. The fast majority of participants evaluated the quality of the programme positively; they were satisfied with the content, the design and user-friendliness. Also, the spoken text was highly valued. These texts were spoken by professional actors, and we believe that this rather unique aspect of the programme has contributed to the positive evaluation. The results were supported by the fact that almost everyone expressed the intention to revisit the website. Useful suggestions were to extend the information, especially about diet and to make it easier for HCPs to turn off sound.

Although the programme was positively evaluated, a minority used the functionalities as intended, including the workbook. Furthermore, according to HCPs and patients the programme is primarily an informative education tool. However, after probing, participants recognized the capacity of the programme to improve communication and self-management. We hypothesize that when the functionalities are better used, by both patients and HCPs, the efficacy of 
DIEP might increase. For example, if workbooks are used for goal setting and discussed with HCPs, this might be helpful in achieving self-management goals (Hurn, Kneebone, \& Cropley, 2006; Van Dam et al., 2003; Wens et al., 2008).

HCPs had difficulties recruiting patients to participate in the one-on-one interview study, often because they did not know whether a patient had worked or not with DIEP. HCPs admitted that they mostly brought the programme only to the attention of some patients, indicating the absence of structural use of DIEP during consultations. For optimal efficacy and a direct effect on selfmanagement, a more adequate implementation and use of the programme is necessary. An active role of HCPs is required for a better implementation; selfmanagement behaviours as outcome measures for evaluating DIEP may then become relevant.

The present study has some limitations. Self-management outcomes were not measured because no improvements could be expected since the experimental group had only access to the programme for two weeks. Future research should investigate long-term effects of DIEP on self-management. Furthermore, in diabetes care, education is an ongoing process in which a patient should have access to education at their convenience. The short access period, together with the high baseline scores may have led to the limited (+ 0.7 ) but significant increase in knowledge. Another limitation is the number of interviews for the user evaluation. However, these interviews with patients and HCPs were used to obtain more explanatory information in addition to information from the online questionnaire among patients. The high number of non-responders of our user evaluation questionnaire, of which we have no data available, should be taken into account in the interpretation of the results.

Despite these restrictions and the limited effects on knowledge, this webbased education tool has high potential. The perceived high quality of DIEP is not only shown in the current study, but also by reactions from people in the field of diabetes care in the Netherlands. After being nominated by representatives of internists, general practitioners, diabetic nurses and DM patients, DIEP won the quality award for diabetes care 2005. Furthermore, in 2006 the Netherlands Institute for Health Promotion and Disease Prevention assessed the quality of diabetes education materials in the Netherlands including DIEP, which was described as a highly promising tool (Looise, Van der Poel, \& Bos, 2006). 


\section{Conclusion}

DIEP can improve knowledge and thereby has the potential to contribute to self-management. Furthermore, participants were highly satisfied with the content, credibility and user-friendliness of the programme. However, the programme is merely seen as an information tool instead of an education tool that offers the opportunity to stimulate patient activation and self-management and therefore needs a better implementation plan.

Online education is easily accessible, can be interactive and education can be adapted (tailored) to individual characteristics based on, for example, learning needs or educational level (Atack, Luke, \& Chien, 2008; Benigeri \& Pluye, 2003). DIEP could be further developed to a more comprehensive source, with more interactive features and tailored information.

\section{Practice implications}

- Most of our participants appreciated the spoken text instead of only written text. Educational websites could use this possibility of Internet more often.

- Due to a close cooperation with the target group and stakeholders we found a high perceived quality of the programme. When developing new interventions, this cooperation should be assured.

- Adequate implementation and use of a new education programme requires an active role of and a clear instruction for HCPs. Therefore, a training for HCPs in how to use the programme in diabetes care is developed: diep@work.

- Continuous development of the programme is necessary for up-to-date information and for processing suggestions for improvement. Therefore the DIEP-foundation is established. The goals of the foundation go beyond diabetes. DIEP can be used as framework for programmes in other countries, as it already was in Belgium, but also for other chronic conditions in which self-management plays an important role.

\section{ACKNOWLEDGEMENTS}

We would like to thank all health care professionals and patients who were involved in the development and evaluation of DIEP. 


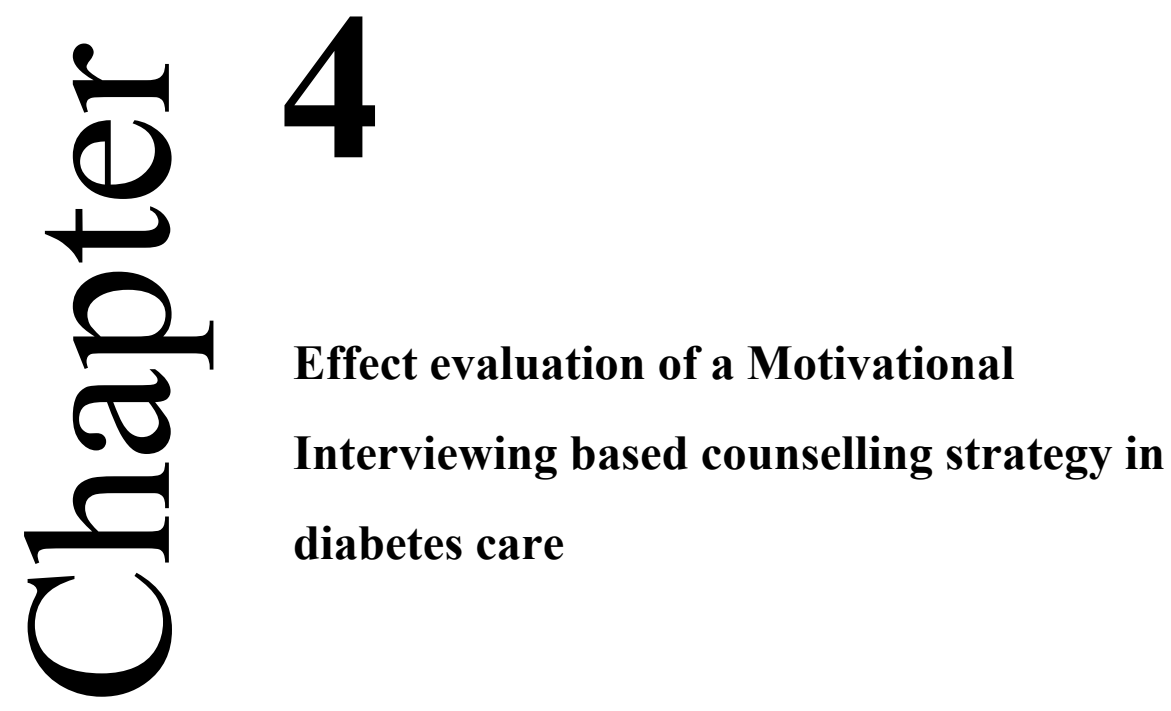

Published as: Heinrich, E., Candel, M.J.J.M., Schaper, N.C., \& De Vries, N.K. (2010). Effect evaluation of a Motivational Interviewing based counselling strategy in diabetes care. Diabetes Research and Clinical Practice, 90, 270-278. 


\section{ABSTRACT}

The present study assessed the effects of a Motivational Interviewing (MI) based counselling training for nurses on clinical, behavioural and process outcomes among diabetes type 2 patients.

The study is an RCT with follow-up measurements after 12 and 24 months. Thirtythree nurses and 584 patients participated. Nurses in the experimental condition received the training; control group nurses were trained after the study. The training consisted of two training sessions, two follow-up meetings, written feedback and three direct feedback sessions. Basic MI-principles and techniques and an MI-based counselling protocol were addressed.

Results indicated disadvantageous effects on fat intake and HDL and advantageous effects on chance locus of control and knowledge. No effects were found on vegetable or fruit intake, physical activity, HbAlc, weight, blood pressure, total cholesterol, LDL, triglycerides, health care climate, quality of life or on self-efficacy.

As in other MI studies, mixed results were found. It would be premature to recommend dissemination of $\mathrm{MI}$ in diabetes care. More studies are needed in realworld settings with health care professionals of the field instead of intensively trained MI interventionists. Knowledge should be gained about adequate training and factors contributing to the implementation of MI in daily practice. 


\section{INTRODUCTION}

The incidence of diabetes type 2 (DM2) worldwide increases rapidly due to changing lifestyles and increased longevity (Deakin, McShane, Cade et al., 2005; Wild et al., 2004). In addition to physical and psychological burden to the patient, the increase in diabetes causes a financial burden to society (International Diabetes Federation, 2008). Besides pharmacological treatment, lifestyle modification (such as adjustment of diet and physical activity) is a crucial element in treatment to prevent or delay the onset of complications (Adler et al., 2000; Norris et al., 2002; UK Prospective Diabetes Study Group, 1998). Because of their daily responsibility for a large number of behavioural choices and activities, patients play a central role in their own treatment. Patients experience difficulties with self-management during daily life, contributing to frequent suboptimal control of risk factors (Clement, 1995; Harris, 2000; Jerant et al., 2005; Vermeire et al., 2005a). To limit the consequences of the increase of patients with DM2, effective ways of patient counselling are urgently needed.

A promising counselling strategy in the treatment of lifestyle problems and disease is 'Motivational Interviewing' (MI). MI is defined as 'a client-centred, directive method for enhancing intrinsic motivation to change by exploring and resolving ambivalence' (p.25) (Miller \& Rollnick, 2002). In contrast to traditional, more paternalistic, counselling styles, MI gives the patients' knowledge and experiences a central role in finding the best behaviour change strategies. The motivation to change should originate from the patient instead of being imposed by the health care professional.

MI was originally developed in the addiction field, but this relatively new counselling style is increasingly being advocated in other health areas such as diet, exercise, and diabetes (Hettema et al., 2005; Miller \& Rose, 2009; Resnicow et al., 2002). Nevertheless, consistent evidence for the effectiveness of MI in these areas is still limited and additional research is indicated (Burke, Arkowitz, \& Menchola, 2003; Dunn, Deroo, \& Rivara, 2001; Resnicow et al., 2002). MI in chronic disease care may require a different approach than in addictive behaviours and the health care providers are different (Resnicow et al., 2002). However, as summarized by Martins and colleagues (2009), MI shows potential for diabetes care. In some of the nine studies reviewed by Martins and colleagues, beneficial effects on glucose levels, physical activity, weight and engagement in dietary changes were found (Martins \& McNeil, 2009). In all studies the intervention consisted of separate (additional) MIsessions aimed at behaviour change instead of MI embedded in usual care. 
In conclusion, MI seems promising for diabetes care but its effectiveness when incorporated in daily practice and not as a separate intervention in addition to usual care is still unclear. Therefore, the present study determined the effects of an MI-based counselling training to nurses on clinical, behavioural and process outcomes in DM2 patients. Nurses were supposed to use the counselling style during usual care.

\section{METHODS}

\section{Study design and procedures}

In the Netherlands, diabetes care is provided mainly in primary care. In most practices patients are seen annually by their general practitioner and quarterly by a "practice" nurse, whose main tasks are monitoring the disease, providing education and lifestyle counselling. These nurses specialize in the care in chronic diseases such as diabetes and COPD, and are supervised by the general practitioner.

General practices were recruited in the southern parts of the Netherlands. In total thirty-three nurses participated. Nurses were asked to list all eligible patients within their practice(s): DM2 for $\leq 5$ years and an age between $40-70$ years. Patients were excluded if they had severe co-morbidity or insufficient command of the Dutch language. All patients were invited by letter to participate in the study. Of the 1517 patients approached, 618 patients (41\%) signed informed consent. Based on the in- and exclusion criteria, 584 patients were included.

This study is a cluster randomized controlled trial. Nurses within a district frequently contact each other and have shared training sessions. To avoid contamination, cluster randomization was chosen. With a computerized randomizer, two districts (18 nurses) were assigned to the experimental condition and two districts (15 nurses) were assigned to the usual care condition. Nurses could not be blinded but assessment of outcomes was done by an independent observer.

The intervention started in autumn 2006, when nurses of the experimental condition were trained. The effects of the intervention were measured among patients. Besides a pre-test before the start of the intervention, post-tests were conducted after twelve and twenty-four months (autumns of 2007 and 2008).

The medical ethics committee of the academic hospital Maastricht and Maastricht University approved the study. 


\section{Intervention}

Nurses in the experimental condition received an MI-based counselling training after baseline measurements. Nurses were supposed to apply the new counselling style during standard quarterly consultations with their patients. Patients of control group nurses received usual care: quarterly consultations with a nurse who did not attend the MI-based training.

During the two years of study, nurses and patients from the experimental and control group had access to a web-based education programme (www.diep.info) that was developed to support self-management by patients. This programme does not only provide information, but also offers tools to support self-reflection, goal setting, problem solving, and active patient participation. The development and content of this programme is described in detail elsewhere (Heinrich et al., 2009).

\section{Training}

Two 51/2-hour sessions were organized to train nurses in an adapted form of MI and the use of the education tool. The MI part was given by a certified trainer. The training consisted of presentations, demonstrations, role-playing and discussions. Nurses received a project folder with information about the study, training material (e.g. cases for role-playing), background information about MI including examples of dialogues between health care professionals and patients, and information about the education tool and how to use it. Furthermore, nurses received instruction charts specifying counselling techniques to support them during consultations. In the week between the two sessions, nurses were asked to read all the MI background information and the information about the education programme (www.diep.info). Three months after the training, nurses received written feedback on two audio-taped consultations. Besides, nurses were visited three times during their work for direct feedback ( $3 \frac{1}{2}$ hours per visit), approximately 6,9 and 11 months after the training. The first time this was done by the MI-trained researcher, while the second and third visits were done by the MI-trained teaching nurse. Additionally, nurses had two opportunities to assemble an afternoon to share experiences and practice together; 8 months and 18 months after the training. In total, approximately 21.5 hours counselling training were given, and 5 hours optional group meetings. 


\section{Adapted motivational interviewing}

The counselling intervention was based on MI (Miller \& Rollnick, 2002) and health behaviour change counselling (Rollnick et al., 1999) and was designed to fit to diabetes care consultations (approximately 20 minutes per consultation).

During the first day of training, basic principles and techniques of motivational interviewing were addressed: expressing empathy, developing discrepancy, rolling with resistance, supporting self-efficacy, getting permission for actions, avoiding the provision of unsolicited advice and/or information, using open-ended questions in stead of closed questions, using reflections and using summaries (Miller \& Rollnick, 2002; Rollnick et al., 1999).

The second day focused on a MI-based counselling protocol, see Figure 4.1, and the use of the education programme. The protocol consisted of the following steps: establish rapport, set agenda together with the patient, assess importance and confidence, explore importance and confidence, summarize positive and negative issues of behaviour change, discuss a plan with the patient, set concrete and realistic goals with a patient if the patient is ready for change and there is a need for goals, summarize the plan and ask if the patient agrees, and finish the consultation appropriately. Strategies that were learned to explore importance and confidence were the 0-10 strategy, the matrix of pro's and con's, and brainstorming about solutions (Miller \& Rollnick, 2002; Rollnick et al., 1999). Counselling skills targeting behaviour change, for example exploring confidence, only had to be used when there was a reason to, like declining medical outcomes. Other skills such as basic principles and techniques should always be used.

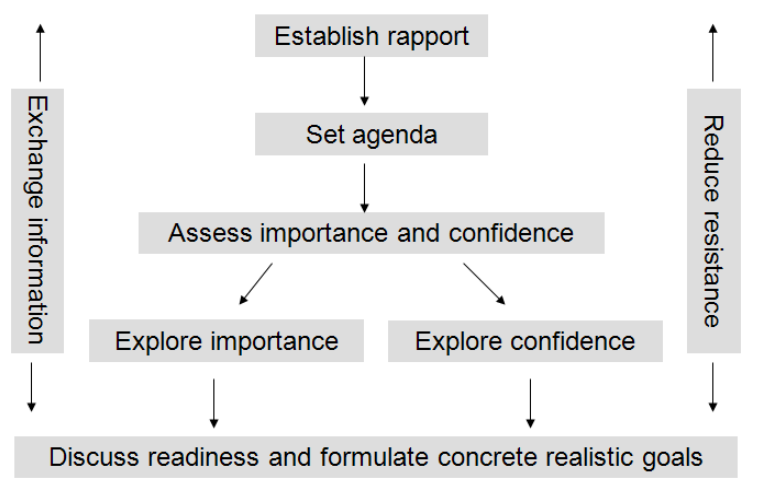

Figure 4.1 Counselling protocol 


\section{Outcome measures}

Measurements took place at baseline, after 12 months and after 24 months. Patients completed a self-administered, written questionnaire with mainly validated scales on self-management behaviours and process outcomes, supplemented with a question about the use of www.diep.info and background variables. The latter included age, sex, ethnicity, educational level, time since diagnosis and living situation (alone or together). Nurses recorded several clinical parameters.

\section{Self-management behaviours}

Fat, fruit and vegetable intakes were measured using validated food frequency questionnaires (FFQ) (Van Assema, Brug, Ronda et al., 2001; Van Assema, Brug, Ronda et al., 2002). The fat FFQ assessed the frequency and amount of 19 products or product groups contributing most to saturated fat intake. Instead of grams fat, a fat score (range 0-80) is calculated because the FFQ does not cover all sources of saturated fat (Van Assema et al., 2001). The FFQ for fruit and vegetable intake assessed the frequency and quantity of fruit, fruit juice and raw or cooked vegetables. Average fruit and vegetable intake in grams per day were calculated (Van Assema et al., 2002).

Two items of the Summary of Diabetes Self-Care Activities scale (SDSCA) were used to assess smoking (smoked during the past seven days; yes/no) and to assess whether the physical activity (PA) standard of $\geq 30$ minutes on at least 5 days a week was met (PA-norm) (Toobert, Hampson, \& Glasgow, 2000).

Additionally, to have a measure of the amount of PA and time spent sedentarily, the Dutch short form of the 'International Physical Activity Questionnaire' (IPAQ) was used (Craig, Marshall, Sjostrom et al., 2003; IPAQ, 2009). This 'last 7 days recall form' assessed walking, moderate-intensity activities, vigorous-intensity activities and sedentary time. For PA, a METminute/week sum score was used as outcome measure (PA-score).

\section{Clinical parameters}

Nurses recorded the following clinical parameters up to 12 weeks old or late at baseline and up to 6 weeks old or late at follow-up: height and weight (for body mass index calculation), blood pressure (BP), glycated haemoglobin (HbA1c), fasting cholesterol (total, LDL, HDL) and triglycerides. 


\section{Process outcomes}

According to the Self Determination Theory (Deci \& Ryan, 1985) increased perceived competence is influenced by perceived autonomy support and improves patient outcomes (Laine \& Davidoff, 1996; Williams, Freedman, \& Deci, 1998). To asses the patient's perception of the degree to which the nurse supported autonomy, the Health Care Climate Questionnaire (HCCQ) was used (15 items, Cronbach's $\alpha=0.95$ ) (Williams, Grow, Freedman et al., 1996). It includes items such as "I feel that my physician has provided me with choices and options". Answers were given on a 1 (strongly disagree) to 7 (strongly agree) scale. Also, perceived competence was assessed by the Diabetes Management Self-Efficacy Scale for people with DM2 (DMSES) (18 items, Cronbach's $\alpha=0.81$ ) (Van der Bijl, Van Poelgeest-Eeltink, \& ShortridgeBaggett, 1999). It includes items such as "I think I am able to overcome high blood glucose levels". The answering options range from 1 (no, certainly not) to 5 (yes, certainly).

Health Locus of Control (HLOC) was measured with the Dutch version of the diabetes specific health locus of control scale for diabetes patients (Halfens, 1985). Three loci of control were distinguished (Wallston, Stein, \& Smith, 1994; Wallston, Wallston, \& DeVellis, 1978; Williams, Rodin, Ryan et al., 1998): Internal, Powerful others and Chance HLOC referring to the beliefs that health outcomes are dependent on one's own behaviour (IHLOC, Cronbach's $\alpha=0.85$ ), on the health care provider (PHLOC, Cronbach's $\alpha=0.75$ ), or that health outcomes are random occurrences (CHLOC, Cronbach's $\alpha=0.72$ ) (Williams, Rodin et al., 1998). Each sub-scale consisted of six items with a Likert-scale ranging from 'fully agree' (1) to 'fully disagree' (6). Subscale scores were sum scores of all six items (range 6-36).

Diabetes specific quality of life (DSQoL) was measured with the Problem Areas in Diabetes Scale (PAID) (20 items, Cronbach's $\alpha=0.95$ ) (Snoek, Pouwer, Welch et al., 2000). This is a self-report measure of diabetes-related distress. Respondents indicate the extent to which a specific aspect of diabetes management is a problem, on a 5-point Likert scale ranging from 'no problem' (0) to 'a serious problem' (4). For example, "Feel discouraged about your treatment".

Additionally, diabetes knowledge was measured by a multiple choice questionnaire (50 items), with a minimum of 0 (zero correct answers) and a maximum score of 50 (all 50 questions correct). To our knowledge there was no validated Dutch knowledge questionnaire, therefore we developed our own. The questionnaire addressed questions about diet (e.g. Does milk contain carbohydrates?), PA (e.g. Through regular exercise, blood glucose levels can 
increase), causes and consequences of diabetes (e.g. Diabetes can cause cardiovascular diseases), and overweight and diabetes (e.g. In patients with diabetes who are overweight, insulin works better than in patients with a healthy weight).

\section{Analyses}

Prior to our study, a power analysis (using Health Locus of Control as criterion variable) led to an initial sample size needed of 120 patients per cell $(p<0.05$, power=0.09); because of expected drop-out we increased the sample size to the final 584 (also because sampling actually was done on the level of the nurses, not the patients).

Data analyses included descriptive statistics of the demographic variables and outcome parameters. Missing data on items of (sub)scales were replaced by the mean score of the individual on the other items of that (sub)scale, but only when no more than $20 \%$ of the values were missing. Scores on the DSQoLscale and the PA-score were not distributed normally and therefore logtransformed. In the case of the HCCQ-score, which was also not normally distributed, transformation did not help. Therefore, next to the continuous HCCQ-outcome, a binary outcome measure based on the median (below or at the median versus above median) was computed. The same was done for the SDSCA-variable which measured the number of days an individual is physically active for at least 30 minutes a day. This variable was transformed into a binary variable for the analysis; 'A minimum of 30 minutes PA on at least 5 days a week: yes / no'.

Since we had multiple measurements and patients were nested within practices, multilevel analyses were conducted to test differences between the experimental and control group at baseline and at follow-up measurements regarding behavioural, clinical and process outcomes, correcting if necessary for influences of time since diagnosis, age, sex, educational level and living situation. Within some practices there was more than one nurse. Patients were not seen solely by one of them and it was impossible to trace which patient was seen by which nurse at all consultations. Therefore it was decided to omit nurses as a level from the analysis. Continuous outcome measures were analyzed with linear mixed regression in SPSS and binary outcome measures with logistic mixed regression in MlwiN (Rasbash, Steele, Browne et al., 2005). In the multilevel approach available cases are included instead of only complete cases (Snijders \& Bosker, 1999), thus enabling an intention-to-treat analysis. An analysis on available cases results in more statistical power. 
Furthermore, the multilevel approach assumes that missings are at random (MAR), that is, they may depend on variables included in the model. This is more flexible than assuming that missings are completely at random (MCAR), in which case they are not allowed to depend on any variable, an assumption made when performing the analyses only on complete cases.

Random slopes and random intercepts were tested first, followed by the fixed effects according to a top-down procedure. The least and non-significant components were deleted step-by-step from the model with a significance level of 0.10 . In the most reduced model, the intervention effects were examined with a 0.05 significance cut-off point. First an overall intervention effect across both follow-up measurements was tested. Only when the overall effect was significant, short and long term effects of the intervention were examined separately. In all analyses, a correction for baseline scores was made.

\section{RESULTS}

\section{Descriptives}

From a total of 36 general practices, 584 patients started in our study of which 537 (92\%) filled out the baseline questionnaire, 447 (77\%) filled out the questionnaire at twelve months follow up and finally $423(72 \%)$ filled out the questionnaire at twenty-four months. Of all patients, 389 (67\%) filled out all three questionnaires and $32(5 \%)$ filled out none.

Concerning clinical parameters, $570(98 \%)$ patients had at least one valid value at baseline, $498(85 \%)$ at twelve months follow up and $462(79 \%)$ at twenty-four months.

The average age of the patients was 59 years $(\mathrm{SD}=5.27)$ and somewhat less than half of them were female (44.9\%). Most patients had lower education (61.7\%), 23.6\% had a medium level of education and $14.7 \%$ had a high educational level. Approximately one quarter (26.4\%) of the patients was diagnosed with diabetes $\leq 1$ year, $47.0 \%$ between $2-3$ years and $26.6 \% 4-5$ years. During the two year follow up, 138 (29.1\%) patients visited the educational programme (www.diep.info) at least once. In the control group, $79 \%$ of the patients never visited the website and another $10 \%$ only visited the website once. For the experimental group these percentages are $73 \%$ and $13 \%$ respectively. At baseline, $77.3 \%$ had an HbAlc level below 7\% (norm score); approximately $41.3 \%$ had a (Body Mass Index) BMI below 28 and $42.2 \%$ had a BMI above 30 . 
Tables 4.1A and 4.1B show baseline scores and the results of the comparison between experimental and control group. No significant differences between the experimental and control group were found in patient characteristics or outcome measures, except for smoking status, physical activity and fat intake. Patients of the experimental group had a lower fat intake (-1.5 points), a lower PA-score (-110 MET-minutes/week), and smoked more often $(+8.8 \%)$.

Table 4.1A Baseline continuous outcome scores and differences between experimental and control group

\begin{tabular}{|c|c|c|c|c|c|}
\hline & \multicolumn{2}{|c|}{ Experimental group } & \multicolumn{3}{|c|}{ Control group } \\
\hline & n & Mean (SD) & n & Mean (SD) & $\mathbf{C}-\mathbf{E}^{\mathbf{a}}$ \\
\hline \multicolumn{6}{|l|}{ Behavioural outcomes } \\
\hline Vegetable intake (grams a day) & 273 & $191.11(84.65)$ & 258 & $198.74(81.94)$ & 7.63 \\
\hline Fruit intake (grams a day) & 273 & $335.76(218.73)$ & 258 & $345.54(201.39)$ & 9.78 \\
\hline Fat intake (points, score 0-95) & 275 & $15.91(6.09)$ & 262 & $17.44(5.62)$ & $1.53 * *$ \\
\hline PA-score (MET-minutes per week) & 153 & $3675.62(4796.12)$ & 139 & $3785.61(3985.86)$ & $109.99 *$ \\
\hline Sedentary time (minutes a day) & 151 & $368.18(200.88)$ & 148 & $329.59(178.15)$ & -38.59 \\
\hline \multicolumn{6}{|l|}{ Clinical outcomes } \\
\hline HbA1c $(\%)$ & 287 & $6.49(0.85)$ & 272 & $6.51(0.74)$ & 0.02 \\
\hline Weight $(\mathrm{kg})$ & 280 & $88.77(17.39)$ & 268 & $88.23(17.19)$ & -0.54 \\
\hline Systolic BP (mmHG) & 280 & $138.63(15.88)$ & 270 & $137.15(15.96)$ & -1.48 \\
\hline Diastolic BP (mmHG) & 280 & $81.95(7.91)$ & 270 & $81.01(8.68)$ & -0.94 \\
\hline Total cholesterol (mmol/l) & 267 & $4.64(0.98)$ & 249 & $4.62(0.88)$ & -0.02 \\
\hline $\mathrm{LDL}(\mathrm{mmol} / \mathrm{l})$ & 267 & $2.60(0.92)$ & 251 & $2.65(0.79)$ & 0.05 \\
\hline $\operatorname{HDL}(\mathrm{mmol} / \mathrm{l})$ & 267 & $1.22(0.32)$ & 249 & $1.17(0.30)$ & -0.05 \\
\hline Triglycerides (mmol/l) & 267 & $1.76(1.01)$ & 252 & $1.69(0.85)$ & -0.07 \\
\hline \multicolumn{6}{|l|}{ Process outcomes } \\
\hline HCCQ-score (score 1-7) & 273 & $6.00(1.11)$ & 260 & $6.21(0.91)$ & 0.21 \\
\hline IHLOC (score 6-36) & 270 & $28.27(4.75)$ & 256 & $28.59(4.43)$ & 0.32 \\
\hline PHLOC (score 6-36) & 271 & $25.78(5.67)$ & 256 & $26.40(5.40)$ & 0.62 \\
\hline CHLOC (score 6-36) & 270 & $15.60(5.70)$ & 256 & $14.76(5.17)$ & -0.84 \\
\hline DSQoL (score 0-80) & 274 & $16.83(13.32)$ & 259 & $16.98(13.92)$ & 0.15 \\
\hline DMSES (score 18-90) & 268 & $73.16(9.42)$ & 259 & $72.26(9.46)$ & -0.90 \\
\hline Knowledge (score 0-50) & 270 & $31.93(7.76)$ & 251 & $33.04(6.27)$ & 1.11 \\
\hline
\end{tabular}

${ }^{\mathrm{a}}$ Control group mean minus experimental group mean; $* p<0.05 ; * * p<0.01$

\section{Intervention effects}

Table 4.2 shows the corrected effect estimates for self-management behaviours (vegetable, fruit and fat intake, physical activity, sedentary time and smoking) after 12 months and after 24 months follow-up. No significant differences between experimental and control group were found except for fat intake in favour of the control group. Correcting for baseline scores, patients of the experimental group had a higher fat intake both at 12 months follow-up and at 24 months follow-up $(p<0.05)$ when compared to the control group. 
Concerning clinical parameters, at 12 months follow-up no intervention effects were found, see Table 4.3. At 24 months follow-up there is a significant difference between groups for HDL-cholesterol in favour of the control group. Compared to the control group, HDL-cholesterol was significantly lower in the experimental group at 24 months follow-up $(p<0.01)$.

The only significant intervention effects in favour of the experimental group were found on process outcomes, as shown in Table 4.2. On both follow-up measurements, 12 and 24 months, scores on the chance HLOC of the experimental group were significantly lower $(p<0.01)$ and their knowledge scores were significantly higher (12 months; $p<0.01,24$ months; $p<0.05)$ compared to the control group.

Table 4.1B Baseline binary outcome scores and differences between experimental and control group

\begin{tabular}{lccc}
\hline & Experimental group: n (\%) & Control group: n (\%) & $\mathbf{C}^{-\mathbf{E}^{\mathbf{a}}}$ \\
\hline Behavioural outcomes & & & \\
PA-norm: & $165(61.1 \%)$ & $154(59.7 \%)$ & 0.01 \\
$\quad$ Meets norm & $105(38.9 \%)$ & $104(40.3 \%)$ & \\
$\quad$ Does not meet norm & $73(26.5 \%)$ & $45(17.3 \%)$ & $0.10^{*}$ \\
Smoking: & $202(73.5 \%)$ & $215(82.7 \%)$ & 0.02 \\
$\quad$ Yes & $137(50.2 \%)$ & $126(48.5 \%)$ & \\
No & $136(49.8 \%)$ & $134(51.5 \%)$ & \\
Process outcomes & & & \\
HCCQ median & median &
\end{tabular}

${ }^{a}$ Differences in proportions between experimental and control group; $* p<0.05$; $* * p<0.01$ 
Table 4.2 Results from the multilevel analyses of behavioural and process measures comparing experimental with control group

\begin{tabular}{|c|c|c|c|}
\hline & $\begin{array}{c}p \text {-value overall } \\
\text { intervention } \\
\text { effect }\end{array}$ & $\begin{array}{c}\text { Corrected effects }{ }^{\mathrm{a}} \text { at } 12 \\
\text { months follow-up exp. vs. } \\
\text { contr. group (95\% CI) }\end{array}$ & $\begin{array}{c}\text { Corrected effects }{ }^{\mathrm{a}} \text { at } 24 \\
\text { months follow-up exp. vs. } \\
\text { contr. group ( } 95 \% \text { CI) }\end{array}$ \\
\hline \multicolumn{4}{|l|}{ Continuous } \\
\hline \multicolumn{4}{|l|}{ Behavioural } \\
\hline $\begin{array}{l}\text { Vegetable intake } \\
\text { (grams a day) }\end{array}$ & 0.34 & $\begin{array}{c}-6.42 \\
(-18.41-5.57)\end{array}$ & $\begin{array}{c}-8.66 \\
(-20.88-3.56)\end{array}$ \\
\hline $\begin{array}{l}\text { Fruit intake } \\
\text { (grams a day) }\end{array}$ & 0.28 & $\begin{array}{c}4.52 \\
(-27.09-36.14)\end{array}$ & $\begin{array}{c}26.79 \\
(-0.84-62.00)\end{array}$ \\
\hline $\begin{array}{l}\text { Fat intake } \\
\text { (points, score 0-95) }\end{array}$ & 0.04 & $\begin{array}{c}0.92 * \\
(0.13-1.72)\end{array}$ & $\begin{array}{c}0.84 * \\
(0.03-1.66)\end{array}$ \\
\hline $\begin{array}{l}\text { PA-score }{ }^{\mathrm{b}} \\
\text { (MET-minutes per week) }\end{array}$ & 0.73 & $\begin{array}{c}0.01 \\
(-0.10-0.12)\end{array}$ & $\begin{array}{c}-0.04 \\
(-0.15-0.08)\end{array}$ \\
\hline $\begin{array}{l}\text { Sedentary time } \\
\text { (minutes a day) }\end{array}$ & 0.16 & $\begin{array}{c}-44.64 \\
(-91.89-2.61)\end{array}$ & $\begin{array}{c}-10.07 \\
(-57.63-37.49)\end{array}$ \\
\hline \multicolumn{4}{|l|}{ Process } \\
\hline HCCQ-score (score 1-7) & 0.12 & $\begin{array}{c}0.09 \\
(-0.11-0.28)\end{array}$ & $\begin{array}{c}0.21 \\
(0.01-0.41)\end{array}$ \\
\hline IHLOC (score 6-36) & 0.19 & $\begin{array}{c}0.71 \\
(-0.09-1.52)\end{array}$ & $\begin{array}{c}0.12 \\
(-0.70-0.94)\end{array}$ \\
\hline PHLOC (score 6-36) & 0.47 & $\begin{array}{c}0.29 \\
(-0.64-1.22)\end{array}$ & $\begin{array}{c}0.59 \\
(-0.36-1.54)\end{array}$ \\
\hline CHLOC (score 6-36) & 0.00 & $\begin{array}{c}-1.56 * * \\
(-2.39--0.73)\end{array}$ & $\begin{array}{c}-1.33 * * \\
(-2.18--0.48)\end{array}$ \\
\hline DSQoL $^{b}$ & 0.35 & $\begin{array}{c}-0.04 \\
(-0.10-0.02)\end{array}$ & $\begin{array}{c}-0.05 \\
(-0.12-0.02)\end{array}$ \\
\hline DMSES (score 18-90) & 0.69 & $\begin{array}{c}0.35 \\
(-0.89-1.60)\end{array}$ & $\begin{array}{c}0.58 \\
(-0.77-1.94)\end{array}$ \\
\hline \multirow[t]{2}{*}{ Knowledge (score 0-50) } & 0.01 & $\begin{array}{c}1.33 * * \\
(0.42-2.24)\end{array}$ & $\begin{array}{c}1.27^{*} \\
(0.31-2.24)\end{array}$ \\
\hline & & $\begin{array}{l}\text { Corrected odds ratios }{ }^{\mathrm{a}} \text { at } 12 \\
\text { months follow-up exp. vs. } \\
\text { contr. group (95\% CI) }\end{array}$ & $\begin{array}{c}\text { Corrected odds ratios at } 24 \\
\text { months follow-up exp. vs. } \\
\text { contr. group (95\% CI) }\end{array}$ \\
\hline \multicolumn{4}{|l|}{ Binary } \\
\hline \multicolumn{4}{|l|}{ Behavioural } \\
\hline PA-norm & 0.50 & $\begin{array}{c}0.76 \\
(0.41-1.38)\end{array}$ & $\begin{array}{c}0.71 \\
(0.38-1.32)\end{array}$ \\
\hline Smoking & 0.65 & $\begin{array}{c}0.79 \\
(0.33-1.86)\end{array}$ & $\begin{array}{c}0.66 \\
(0.28-1.59)\end{array}$ \\
\hline \multicolumn{4}{|l|}{ Process } \\
\hline HCCQ (score 0-1) & 0.92 & $\begin{array}{c}1.12 \\
(0.59-2.15)\end{array}$ & $\begin{array}{c}1.00 \\
(0.52-1.93)\end{array}$ \\
\hline
\end{tabular}

${ }^{a}$ Corrected for baseline differences on the outcome variable and covariates; $* p<0.05 ; * * p<0.01$

${ }^{\mathrm{b}}$ Log-transformed scores 
Table 4.3 Results from the multilevel analyses of clinical measures comparing experimental with control group

\begin{tabular}{|c|c|c|c|}
\hline & $\begin{array}{c}p \text {-value overall } \\
\text { intervention } \\
\text { effect }\end{array}$ & $\begin{array}{l}\text { Corrected effects }{ }^{\mathrm{a}} \text { at } 12 \\
\text { months follow-up } \\
\text { exp. versus contr. group } \\
(95 \% \mathrm{CI}) \\
\end{array}$ & $\begin{array}{c}\text { Corrected effects }{ }^{\mathrm{a}} \text { at } 24 \\
\text { months follow-up } \\
\text { exp. versus contr. group } \\
(95 \% \text { CI })\end{array}$ \\
\hline HbA1c (\%) & 0.44 & $\begin{array}{c}0.02 \\
(-0.10-0.14)\end{array}$ & $\begin{array}{c}0.09 \\
(-0.05-0.23)\end{array}$ \\
\hline Weight (kg) & 0.66 & $\begin{array}{c}-0.03 \\
(-0.79-0.73)\end{array}$ & $\begin{array}{c}0.33 \\
(-0.57-1.23)\end{array}$ \\
\hline Systolic BP (mmHG) & 0.63 & $\begin{array}{c}-1.09 \\
(-3.91-1.72)\end{array}$ & $\begin{array}{c}-1.29 \\
(-4.17-1.59)\end{array}$ \\
\hline Diastolic BP (mmHG) & 0.26 & $\begin{array}{c}0.24 \\
(-1.31-1.80)\end{array}$ & $\begin{array}{c}1.27 \\
(-0.32-2.86)\end{array}$ \\
\hline Total cholesterol (mmol/l) & 0.25 & $\begin{array}{c}0.06 \\
(-0.09-0.22)\end{array}$ & $\begin{array}{c}-0.08 \\
(-0.56-0.10)\end{array}$ \\
\hline $\mathrm{LDL}(\mathrm{mmol} / \mathrm{l})$ & 0.77 & $\begin{array}{c}0.05 \\
(-0.08-0.18)\end{array}$ & $\begin{array}{c}0.03 \\
(-0.13-0.19)\end{array}$ \\
\hline $\operatorname{HDL}(\mathrm{mmol} / \mathrm{l})$ & 0.01 & $\begin{array}{c}-0.02 \\
(-0.06-0.01)\end{array}$ & $\begin{array}{c}-0.07 * * \\
(-0.12--0.03)\end{array}$ \\
\hline Triglycerides (mmol/1) & 0.97 & $\begin{array}{c}-0.02 \\
(-0.16-0.13) \\
\end{array}$ & $\begin{array}{c}-0.02 \\
(-0.17-0.14) \\
\end{array}$ \\
\hline
\end{tabular}

${ }^{a}$ Corrected for baseline differences on the outcome variable and covariates; $* p<0.05 ; * *<0.01$

\section{DISCUSSION}

The present study was an evaluation of an MI-based counselling strategy embedded in usual diabetes care. Results indicate no major intervention effects on outcome measures, although minor differences between groups were found at follow-up measurements for fat intake, HDL-cholesterol, chance health locus of control and knowledge.

Concerning fat intake and HDL-cholesterol an adverse effect was found. At baseline, the control group had a higher fat intake compared to the experimental group and a possible explanation for the decrease in fat intake in the control group could be that the subjects with a higher fat intake at baseline became more aware of their unhealthy behaviour as a result of filling out the baseline questionnaire. Alternatively, the differences at post-tests might be a simple case of regression to the mean. Although an intervention effect was found on HDL-cholesterol, no effects were found on total or LDL-cholesterol. Besides, the corrected effect of $-0.07 \mathrm{mmol} / \mathrm{l}$ is so small that it might be seen as clinically irrelevant.

Advantageous effects were found on chance HLOC (CHLOC) and knowledge. The effect on CHLOC indicates that people in the experimental group were less inclined than the control group to relate their diabetes control 
to chance factors. Empowered patients are considered to perceive more control over their diabetes, which has been associated with better self-management outcomes (Surgenor, Horn, Hudson et al., 2000; Watkins, Connell, Fitzgerald et al., 2000). However, we found a decrease in CHLOC, but we did not find an increase in internal HLOC nor did we find a change in powerful others HLOC. Concluding that our intervention contributed to patient empowerment would therefore be premature and also inconsistent with the lack of effect on selfmanagement outcomes. Concerning knowledge, due to the intervention patients might have had a more active role during consultations. As a result, patients possibly felt more confident to ask questions and this might have improved knowledge. However, the corrected effects of 1.33 and 1.27 mean a difference of $<1.5$ points on a scale of $0-50$ : a rather modest effect in terms of absolute magnitude.

All in all, based on our study we cannot conclude that our MI-based counselling strategy offers advantages over usual care. Previous research of MI in diabetes care is limited and has shown mixed effects. In two studies where MI-sessions given by a MI-trained psychologist were added to a weight loss intervention for female DM2 patients, effects of MI were found on glucose control (Smith-West, DiLillo, Bursac et al., 2007; Smith, Heckemeyer, Kratt et al., 1997), on blood glucose monitoring (Smith et al., 1997) and on weight (Smith-West et al., 2007). In another study where three individual MI sessions and three follow-up telephone calls were added to usual DM care, no effects were found on glucose control or BMI, and inconsistent results were found for self-management behaviours (Clark, Hampson, Avery et al., 2004a; Clark, Hampson, Avery et al., 2004b). Ismail and colleagues (2008) evaluated four MI-sessions given by a diabetes nurse and added to usual care. They did not find any effect on glucose control, BMI, QoL or on self-management behaviours (Ismail, Thomas, Maissi et al., 2008).

There are several factors in our study that might have influenced the outcomes. First of all: the interventionist. One of the differences between our study and previous studies is that our intervention was delivered by trained nurses instead of a psychologist. Although Rubak and colleagues (2005) concluded that effectiveness was not related to the counsellor's educational background as medical doctor or psychologist, they did find that only 5 out of 11 studies that involved other health workers as MI-counsellors (e.g. nurses) were effective (Rubak, Sandbaek, Lauritzen et al., 2005). Perhaps we would have found better results when patients consulted a trained psychologist instead of a trained nurse. However, we aimed to embed MI in usual care, by usual health professionals. 
A second factor that might have influenced the outcomes is that the counselling strategy had to be applied during regular consultations instead of separate MI-sessions in addition to usual care. This is especially difficult due to the different agendas of patients and nurses. Nurses' agendas may be influenced by treatment protocols and/or by reimbursement systems (Emmons \& Rollnick, 2001). In usual diabetes care, not all consultations are dedicated to self-management. Self-management (including lifestyle changes) is only one topic besides other topics such as discussing side effects of medication, insurance issues, and eye examinations. In a study where MI was embedded in diabetes care, outcomes of consultations with a MI-trained dietician were compared to outcomes of consultations with a non-MI-trained dietician. As in our study, limited intervention effects were found. No effects were found on $\mathrm{HbA1c}$, BMI, fruit and vegetable consumption except on fat consumption (Brug, Spikmans, Aartsen et al., 2007). Accordingly, more studies are needed to know what the implications are of MI embedded in diabetes care delivered by patients' usual care givers.

A third study characteristic that might have influenced the outcomes is the intervention delivery dose (Resnicow et al., 2002). If nurses were unable to adequately implement the strategy, there is a risk of a type III error (Basch, Sliepcevich, Gold et al., 1985). This possible risk should be taken into account when interpreting the findings of our study. Training nurses to change their behaviour might be as challenging as helping patients to change (Rollnick, 2001). Perhaps the nurses should have been trained more intensively to practice the MI-based counselling strategy in daily work. We offered two training sessions, two follow-up meetings, written feedback once and three direct feedback sessions during daily work which is more than what is done in other studies where courses often have a workshop format without any kind of follow-up (Madson, Loignon, \& Lane, 2009). The question is how realistic it is to demand even more training time than what we did. A possible solution to this would be to incorporate training in MI counselling strategies during regular courses for students. Training intensity is only one aspect of the delivery dose. Also the total time spent on self-management changes during our intervention period may have been too limited. In accordance with the Dutch diabetes care guidelines, the patients in our study had 3 to 4 consultations per year in which all aspects of diabetes treatment had to be addressed. Previous MI studies in diabetes care offer specific MI sessions to patients which automatically results in more exposure time (Martins \& McNeil, 2009). Review studies have shown that even small doses of MI ( $<15$ minutes) can be effective (Rubak et al., 2005) but that higher treatment doses ( $>60$ minutes) tend to increase the effectiveness 
(Burke et al., 2003; Rubak et al., 2005). Our intervention should be considered as low intensity MI and perhaps more consultations including MI-counselling were needed to be effective. Additional research is needed in this area as the effect of delivery dose is still inconclusive (Martins \& McNeil, 2009).

Fourth, the intervention content might have played an important role. We did not implement MI as it was originally developed in the field of substance use. We created an adapted form (AMI) suitable to quarterly consultations in DM care. However, a previous review and a meta-analysis specifically aimed at AMI-interventions showed beneficial effects (Burke et al., 2003; Dunn et al., 2001). Of course, our adaptation may have been suboptimal although we based it on earlier experiences.

A final study characteristic that might have been of importance is our patient population. We did not specifically aim to include people with suboptimal outcomes and looking at the clinical outcomes and some behavioural outcomes our population seems relatively healthy. Approximately three quarters had a baseline $\mathrm{HbAlc}$ of $<7 \%$, the average systolic blood pressure was below $140 \mathrm{mmHG}$ and lipid profiles were near normal as well. Furthermore, self-reported vegetable and fruit intake were sufficient at baseline and $60 \%$ of the population reported to meet the physical activity norm. Also, the average baseline score on the measure for autonomy support was 6.1 on a scale of 1-7. According to the self-determination theory, autonomy supportive health care providers can influence a patient's autonomous motivation and thereby improve health outcomes (Williams, Freedman et al., 1998). However, at baseline patients already perceived their health care provider as autonomy supportive, indicating little improvement to expect. These data show a possible ceiling effect; little improvement may be expected from the intervention. On the other hand, more than $50 \%$ had a baseline BMI $>28$, and $40 \%$ did not meet the PA-norm, leaving ample room for improvement. Also, improvement of health locus of control dimensions, quality of life and self-efficacy could be made. Additional analyses to test the effects of baseline Hbalc and BMI on outcomes did not show significant interactions between intervention effects and baseline values. The apparent healthy baseline outcomes may be influenced by medication effects on clinical outcomes, which we unfortunately did not measure, and social desirability effects on dietary intake and PA. For example, it is known that fruit and vegetable intake is insufficient among Dutch adults (De Hollander, Hoeymans, Melse et al., 2006). Although a ceiling effect should be taken into consideration when interpreting our findings, it cannot fully explain the lack of effectiveness. Another population characteristic is our sample size but a lack of power seems unlikely when we compare our sample 
size to other studies (Keers, Bouma, Links et al., 2006; Martins \& McNeil, 2009).

Our results indicate a need for further research towards MI-based counselling embedded in usual care and assessment of factors influencing the use of such counselling strategies for a better understanding of the applicability of MI-interventions in diabetes care. Evaluations addressing these issues and a description of training methods are often lacking (Dunn et al., 2001; Miller, 2001; Rollnick, 2001). We gave a comprehensive description of the training and intervention. In addition to the current study, we evaluated tape-recorded consultations. These tapes show that some skills were implemented (e.g. agenda setting and assessing importance and confidence) but others were not (e.g. reflective listening and exploring importance and confidence). The implementation study also showed the importance of direct feedback on nurses' performances in daily practice and this study will be reported in the near future.

The present study had its limitations. There are no data available of patients or nurses who refused to participate. Therefore, we may have had a selection of nurses who have a special interest in new counselling techniques, which would limit external validity. However, with less motivated nurses it would have been even harder to find intervention effects. Furthermore, to avoid contamination we randomized on district level rather than on practice or patient level. Therefore, we corrected for possibly relevant baseline differences in all analyses. Additionally, we were not in control over counselling behaviours of control group nurses. For example, nurses were allowed to use the web-based education programme, but this limitation is inherent in the practice-based character of our study. Finally, behavioural outcomes were mostly measured by self-report questionnaires, which may have caused bias (Bartholomew et al., 2000), but analyses of more objective clinical outcomes resulted in similar conclusions.

In sum, based on our study and what is known from other studies so far, it would be premature to recommend dissemination of MI in daily practice of diabetes care. More studies are needed in real-world settings with health care professionals of the field instead of intensively trained MI interventionists. Knowledge should be gained about adequate training and skill levels of health care professionals as well as about factors contributing to the implementation level in daily practice, such as characteristics of the general practices, patient populations, MI, and the health professionals themselves. 


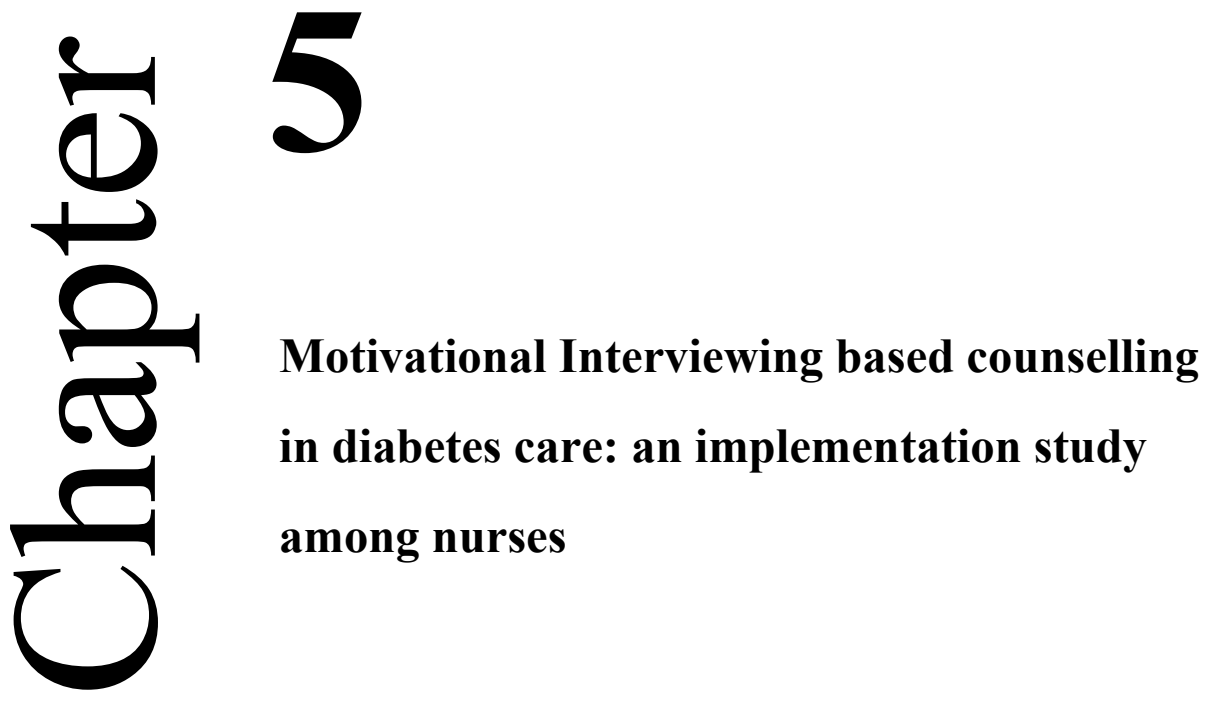

Re-submitted for publication as: Heinrich, E., Mesters, I., Schaper, N.C., \& De Vries, N.K. Motivational Interviewing based counselling in diabetes care: an implementation study among nurses. 


\begin{abstract}
A promising counselling strategy in the treatment of lifestyle problems and disease is Motivational Interviewing (MI). MI was originally developed in the addiction field, but is increasingly being advocated in other health areas such as diet, exercise, and diabetes. Current clinical trials seldom include a fidelity check of the actual use of MI and often lack a clear description of MI training procedures. The present implementation study assessed the level of use of an MI counselling method by nurses in diabetes consultations who attended a Motivational Interviewing based training, and explored factors influencing the use of the MI method.

A total of 18 nurses who attended an MI-based counselling training program, audio-taped consultations 3 months, 1 year and 2 years after the training. Additionally, 15 other nurses served as reference group; they audiotaped consultations after 1.5 years and attended the training two years later than the first group. The level of MI use was evaluated by scoring audio-taped consultations. Changes over time in the first training group and differences between the first training group and the reference group were assessed. Factors influencing the implementation of MI in diabetes care were explored by conducting telephone interviews $(n=26)$ with nurses of both groups (after the reference group had also been trained).

Audio-tape scores showed that our training program improved five out of twenty skills: agenda setting, assessing importance and confidence, summarizing, and avoiding unsolicited advice and/or information. However, interview data indicated that nurses perceived themselves as performing better on all twenty items. The most important implementation factors mentioned were: the amount of training and feedback, reminders, support from supervisors and colleagues, time per patient, type of patient and perceived workload.

Changing health professionals' behaviour is difficult and requires sustained training efforts. Examples of implications for future training activities and research are: adding reminders to patient data recording systems, organizing peer feedback, including self-reflective elements, and combining external observation and self-report measures of practitioner behaviour.
\end{abstract}




\section{BACKGROUND}

Behaviour change is a necessity for many chronically ill people, and health care professionals face the challenging task of guiding patients in changing unhealthy behaviour and overcoming their tendency to downplay health risks when confronted with personally threatening information (Leffingwell, Neumann, Babitzke et al., 2007), such as the risk of cardiovascular diseases for patients with diabetes. A specific clinician counselling style which may be helpful in overcoming this defensive response is Motivational Interviewing (MI).

MI is a directive, client-centered counselling style to elicit behaviour change by helping clients explore and resolve ambivalence about behaviour change (Miller \& Rollnick, 2002). The strongest evidence of MI effectiveness in changing lifestyle behaviours has been found in clinical settings in the field of substance abuse (Burke et al., 2003; Hettema et al., 2005). Nowadays, MI is applied in various settings (Britt, Hudson, \& Blampied, 2004; Hettema et al., 2005; Miller \& Rose, 2009), for example to target chronic disease behaviours such as diet, physical activity and treatment adherence (Resnicow et al., 2002). Applying MI to other settings requires adaptation of the original MI method to suit specific circumstances (Emmons \& Rollnick, 2001; Rollnick, 1996), and more research into the clinical utility of MI in different settings is needed (Martins \& McNeil, 2009; Rubak et al., 2005).

Current clinical trials seldom include a fidelity check of the actual use of MI (Burke et al., 2003; Martins \& McNeil, 2009) and often lack a clear description of MI training procedures (De Blok, De Greef, Ten Hacken et al., 2006) Rubak and colleagues (2006) describe one of the few implementation studies of MI in diabetes care, showing that general practitioners (GPs) had changed their behaviour in daily practice after an MI course (Rubak, Sandbaek, Lauritzen et al., 2006). Another implementation study, involving MI-based training for GPs to improve patients' asthma medication adherence, found positive changes in GPs' attitudes and self-confidence (Broers, Smets, Bindels et al., 2005). The GPs also reported using the skills during their consultations. Both studies, however, used self-reported data, which tend to be less reliable compared to objective measures like observations (Miller \& Mount, 2001).

Implementing MI interventions in 'real world' settings with clinicians who have their own routines is difficult (Carroll, Farentinos, Ball et al., 2002). Crucial factors for successful uptake by clinicians may include not only training methods but also other factors, for instance those related to the organization (general practice), the innovation (MI), and the clinicians 
themselves (Fleuren, Wiefferink, \& Paulussen, 2004; Steckler, Goodman, McLeroy et al., 1992).

We developed a counselling intervention for nurses in primary diabetes care, based on MI and health behaviour change counselling (Miller \& Rollnick, 2002; Rollnick et al., 1999). The MI-based counselling intervention was developed to improve health outcomes of patients with type 2 diabetes (DM2). The effects of this intervention on patients' health outcomes were studied in a cluster-randomized controlled trial which is beyond the scope of the current article.

The aim of the current implementation study was to describe the MI-based counselling training, to assess the level of use of the MI method in diabetes consultations after attending the training program and to explore factors that influenced the use of this method.

\section{METHODS}

\section{Procedures}

In the Netherlands, so-called 'practice nurses' in primary care are responsible for the daily care for DM2 patients primary care, under the supervision of a GP. These practice nurses specialize in chronic care, such as diabetes, and see DM2 patients three to four times a year (Nederlands Huisartsen Genootschap, 2006; Nederlandse Diabetes Federatie, 2010). The nurses' main tasks are to monitor the disease and provide education and lifestyle counselling, which is why we focused on practice nurses to implement MI counselling.

Nurses were recruited in the southern parts of the Netherlands. A first introduction was given by telephone, followed by written information about the project and if necessary, this information was clarified during a personal visit of the researcher. In total, thirty-three nurses voluntary participated in our project. Since nurses working in the same district frequently consult each other and share training sessions, nurses were randomized at district level to avoid contamination. After randomization, nurses in the experimental condition $(\mathrm{n}=18)$ attended MI-based counselling training in November 2006. Next, nurses had to apply MI counselling during routine quarterly consultations with their patients. Control group nurses $(\mathrm{n}=15)$ attended the same training approximately two years later, at the end of our study (January 2009).

The medical ethics committee of the Maastricht University Hospital and Maastricht University approved the study (MEC 05-132) and patients' written informed consent was obtained. 


\section{Intervention description}

\section{Training program}

The intervention consisted of a basic group training course followed by additional individual and group training activities. A timeline of all activities and measurements is shown in Figure 5.1.

The basic training course consisted of two 5.5-hour sessions (one-week interval) to train nurses in MI, and was given by a certified MI trainer, a teaching nurse and a researcher. Training involved presentations, video and live demonstrations, role-playing and discussions. Nurses received a project folder with information about the study, training materials (e.g. cases for roleplaying), and background information about MI based on a Dutch translation of the 'Health Behavior Change' book by Rollnick and colleagues (Rollnick et al., 1999). Nurses were asked to read the MI background information in the week between the two sessions. As support during consultations, nurses also received 'reminder cards', summarizing some main counselling techniques (agenda setting, discussing importance and confidence, goal setting, summarizing, using open-ended questions and reflections).

Additional training activities involved individual feedback and follow-up group meetings. Nurses received personal written feedback on two audio-taped consultations, three months after the basic training to support self-efficacy and to indicate possible areas for improvement. Approximately 6, 9 and 11 months after the basic training, nurses received immediate personal feedback during practice visits. The first time this was done by the MI-trained researcher, while the second and third visits were done by the MI-trained teaching nurse. Practice nurses were observed during their regular consultations and a short evaluation took place after every consultation. Special attention was paid to the following skills: agenda setting, exploring importance and confidence, goal setting, providing summaries, using open-ended questions and using reflections. Additionally, practice nurses had two opportunities to get together for an afternoon to share experiences and practice together, 8 and 18 months after the training course. 


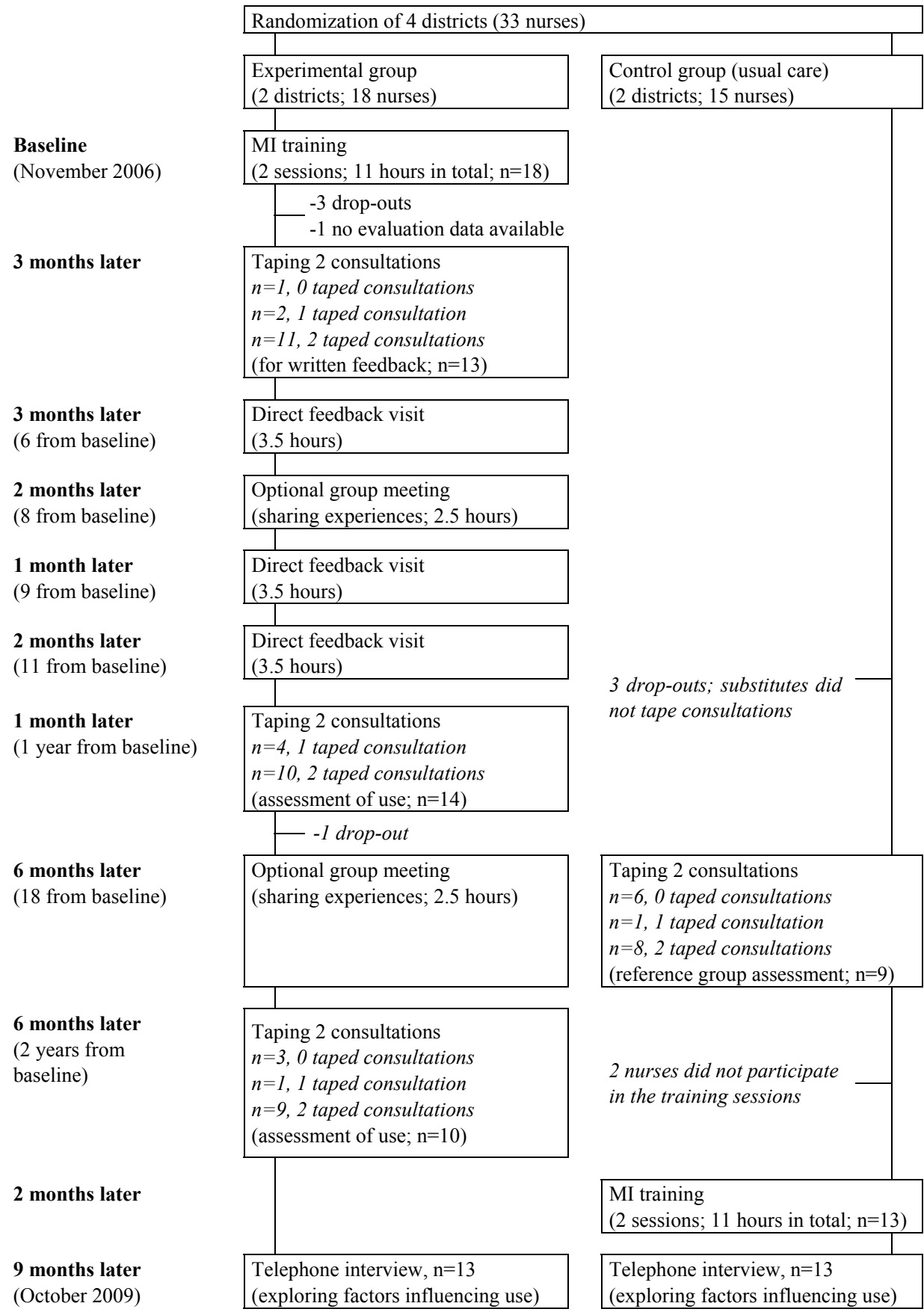

Figure 5.1 Flowchart of intervention activities and measurements 


\section{MI based counselling}

The first day of the training course addressed basic principles and techniques of motivational interviewing: using open-ended questions vs. closed questions, using summaries, using reflections, expressing empathy, getting permission for actions, avoiding unsolicited advice and/or information, supporting selfefficacy, developing discrepancy, and rolling with resistance (Miller \& Rollnick, 2002; Rollnick et al., 1999).

The second day focused on an MI protocol, see Figure 5.2 (Heinrich et al., 2009; Miller \& Rollnick, 2002; Rollnick et al., 1999). Before creating a plan for patients, advantages and disadvantages of behaviour change are summarized. If patients are ready for change, concrete and realistic change goals are set. Appropriate ways to end the consultation were also addressed. Strategies that were learned to explore importance and confidence were the 010 ruler, the matrix of pros and cons, and brainstorming about solutions (Miller \& Rollnick, 2002; Rollnick et al., 1999).

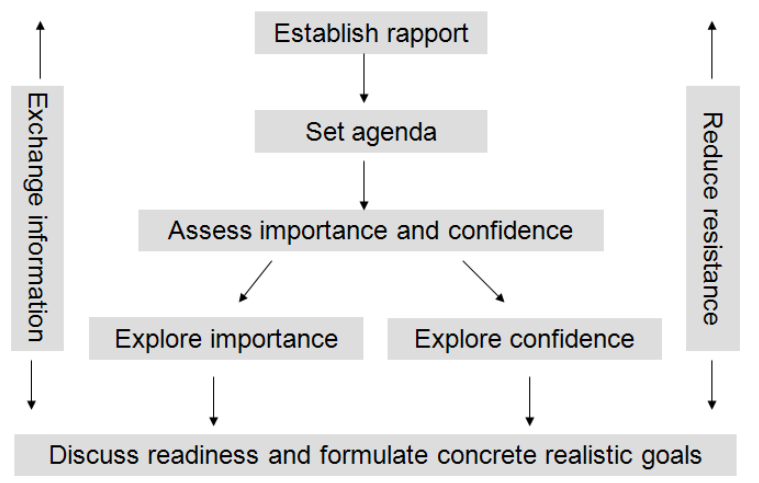

Figure 5.2 Counselling protocol

\section{Measures}

To explore the nurses' level of MI use, audio-taped consultations by nurses of the experimental and control group were evaluated using a rating form. Since our training content differed from MI as originally developed by Miller and Rollnick (Miller \& Rollnick, 2002), an adjusted rating form was produced to assess the level of use of the skills we addressed during the training (see Table 5.1 for the rating form items and Appendix 5.1 for a short manual). The rating 
form consisted of 20 items on specific skills and one item reflecting the overall impression of the consultation. The consultations of the experimental group were taped 3 months, 1 year and 2 years after the basic training course. Nurses of the control group (usual care) served as a reference group. They were asked to tape two consultations approximately 1.5 years after enrolment.

Factors that nurses thought contributed to the use of MI were explored in telephone interviews. This was done after the control group nurses had also been trained (two basic training sessions). The interviews included control group nurses and nurses of the experimental condition. Each interview started with a structured part in which nurses gave themselves a score for all items of the rating form, followed by an exploratory part about factors the nurses considered important. The final part was semi-structured; nurses were asked to rate the importance of specific implementation factors mentioned spontaneously and by the interviewer. Factors presented were based on previous research (Fleuren et al., 2004; Steckler et al., 1992) and included the following categories: characteristics of the innovation, like compatibility with other practice routines and complexity (e.g. To what extent do you think MI can be used in the daily care of DM2 patients?); characteristics of the training (e.g. To what extent do you think you have had enough training?); characteristics of the organization (e.g. To what extent is attention given to counselling methods in the practice you work for?); and characteristics of the health professionals (e.g. To what extent does motivational counselling fit with your own work routine?).

\section{Analyses}

Nurses were asked to tape two consultations per measurement. These consultations were evaluated by two independent trained research assistants who were blinded for condition. Twenty-one items were scored (Table 5.1). An average score was calculated when one assistant scored ' 1 ' and the other scored ' 2 '. The same was done for scores of ' 4 ' versus ' 5 '. In case of other differences, the assistants had to discuss these discrepancies to decide on a final score. Subsequently, an average score per item per measurement was calculated for each nurse. If only one taped consultation was available, the scores for this consultation were used. Next, average group scores were calculated per item for each measurement. Because of the limited number of nurses, nonparametric tests were used to analyze the differences between measurements, using the Wilcoxon Signed Rank test for the differences between 
measurements within the experimental group and the Mann-Whitney U-test for differences between the experimental and control group.

Telephone interviews were analyzed by transcribing taped interviews and then analyzing them by means of the QSR Nvivo 2.0 qualitative data analysis software (www.qsrinternational.com). A thematic approach was used, in which the interview questions were the framework of the analysis. In each transcript, relevant sections were assigned to the appropriate topics. The results were clustered into the four categories described in the method section. The results were presented to the participants, and interpretations of quotes were adjusted if necessary.

\section{RESULTS}

The experimental group consisted of 18 nurses who had attended the basic training course. Three of them got another job almost immediately after the training and one nurse did not audio-tape any consultations. They were excluded from this study. One nurse dropped out before the final measurement two years after the basic training because she retired. The 14 nurses in the current study all attended the two basic training sessions and received 3 feedback visits. Attendance at the two follow-up meetings was lower, but all nurses except one attended at least at one session. See Figure 5.1 for participant flow and taped consultations per measurement.

The control group consisted of 15 nurses. Three of them got another job and the substitute nurses did not tape consultations. At the measurement 1.5 years after the basic training, consultations of nine nurses were evaluated; three nurses failed to audio-tape.

Twenty-six nurses participated in the telephone interviews (13 per group). The interviews lasted 35 minutes on average. The control group nurses participating in this part of the study had all attended the two basic training sessions. A description of the interview results was sent to the participants to check if the interviews were correctly interpreted. All 20 nurses who responded to the description agreed with the interpretation.

\section{Level of use}

Table 5.1 lists the mean scores per item per measurement, including the observation scores based on taped consultations and the scores nurses gave themselves in the first part of the telephone interviews. 


\section{Experimental group (observation scores)}

Three months after the basic training, the items 'establish rapport' and 'express empathy' had at least a mean score of 'good', while 'thank the patient, end consultation appropriately', 'get permission for actions', 'avoid unsolicited advice/information', 'roll with resistance', and 'support self-efficacy' were implemented to some extent but not sufficiently. All other items, including motivation building strategies like exploring importance and confidence, developing discrepancy and the overall impression, had a score below three, indicating poor or insufficient use.

After the additional training activities one year after the basic training course, the use of 'set agenda', 'summarize plan and ask if the patient agrees', and 'use summaries' improved, although summary use still had a mean score below three ('to some extent'). The overall impression also improved, to a mean score of 3.0. Scores on other items did not change significantly.

Compared to the one year measurement, the measurement two years after the training found that the use of agenda setting had deteriorated but setting concrete, realistic goals had improved. Agenda setting was still significantly better than immediately after the basic training and the assessment of confidence significantly improved over time. Scores on other items remained largely the same.

\section{Experimental versus control group (observation scores)}

After one year, when the training activities for the experimental group had been completed, the experimental group scored better than the reference group on five items: 'set agenda', 'assess importance', 'summarize plan and ask if patient agrees', 'avoid unsolicited advice/information', and 'use summaries'. Their score for 'overall impression' was also higher.

\section{Observation versus self-report score}

Nurses gave themselves higher scores on all items compared to the observation scores. The average observation score was 2.90 , whereas the average selfreport score was 4.14 , indicating the difference between 'to some extent' and 'good' use of the counselling style.

During the interviews, nurses said that the taped consultations may not have been representative of their consultation skills, since audio taping negatively influenced their work routine by making them a bit nervous, and making them select 'easy' patients or those who only came for a checkup visit. Factors contributing to the use of the method

The quotations below are based on nurses' statements. 


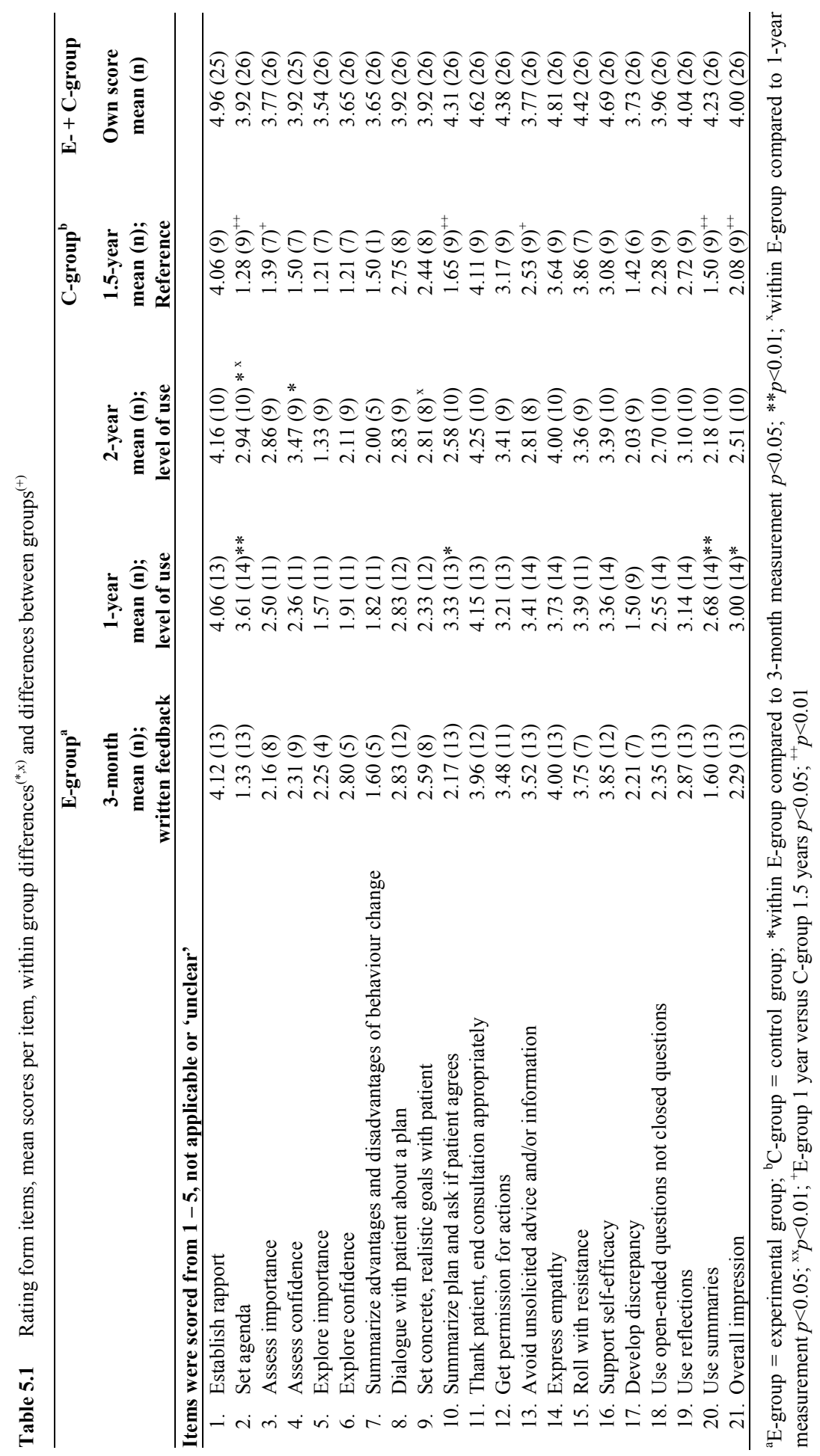




\section{Characteristics of motivational counselling}

Nurses described distinct advantages of motivational counselling compared to their former counselling methods. It helped nurses to structure their consultations and to improve their time management, and provided more clarity about the aim and content of a consultation by setting the agenda and summarizing what had been said. Also, it helped them understand patients better and discover what they want. Patients would be more actively involved and have a better understanding of the disease. 'Normally, I always wanted to present the solution to patients. But now I see that patients start working themselves.' One third of the nurses said that recognizing the advantages stimulated them to use the method. After prompting, most nurses said that difficulty of MI was not an issue. 'I don't think it's the level of difficulty. I think it's more about me. You need discipline to learn it and to change your routine.'

The most frequently mentioned disadvantage of the MI strategy was that it takes too much time. 'Sometimes I do want to explore importance and confidence, but you don't know what will come and if you'll be able to complete the discussion within this consultation.' Some nurses mentioned that this style can be somewhat overdone or naggish and is not suitable for all patients. Few nurses also said that MI is easier to use in smoking cessation consultations, since there is only one specific topic on the agenda.

\section{Characteristics and perceptions of training activities}

The quality of the training program was generally evaluated as good. Nurses mentioned the pleasant atmosphere during the meetings. The 'reminder cards' and the direct feedback visits were particularly useful according to many nurses. 'The reminder card on my desk beside my computer really worked. If you use this for weeks, you know how to do it.' 'When someone visited me and listened to my consultations, we had the opportunity to discuss the specific case and what I could have done differently.'

The most frequently mentioned potential improvement was an increase in number of training sessions, to reinforce the content, and more feedback moments. "If there had been more training sessions it would have left a more lasting recall of the content. Just like our patients, we need that too.' 'Feedback on consultations is very helpful because now you're doing it your own way, but who can tell me if I'm doing it right?' 


\section{Characteristics of the practice}

All nurses stated that they were able to use MI for more than $50 \%$ of their general practice patients, and almost half of all nurses even reported a percentage of $80 \%$ or higher. Nurses thought it would be difficult to use the method for people with a low socio-economic status, with limited understanding of diabetes, and for immigrant patients with a language barrier. It would also be difficult for patients with longstanding and well-managed diabetes, because they are used to a certain type of counselling and to consultations focusing on checking the current status, rather than lifestyle changes. 'The MI protocol focuses on behaviour change, but at a certain point in time, you're done with that. Then you only ask if everything is going well with their diet and exercise, without exploring these things further, especially when the medical parameters are satisfactory.' Patients often have to get used to this type of counselling. 'Some people don't understand what I'm doing, especially those who have known me for some time. They think: What's she doing? People expect another style and it's difficult not to go along with that.' Some patients, mostly the elderly, would need a more paternalistic approach. A few nurses also expressed difficulties with patients who resist lifestyle changes; 'I know my patients and for some you just know it's not going to work, unfortunately.' However, the majority of nurses thought that MI fitted in with their current consultations.

The most commonly mentioned practice-related barriers were workload and the time required for patient data recording systems, which are a priority for general practitioners and insurance companies, but which do not always fit in with the MI protocol. 'We have to record so many things (e.g. blood pressure and weight) and it's the output of these forms that is discussed. No one cares whether you've set concrete and realistic goals.' Regarding workload, nurses tended to revert to their old routines when perceived workload increased and they did not take the time to use the new motivational counselling style. 'Lately, it's been so busy with all the new things in our practice. I noticed that I'd fallen back into my old routine. I don't ask patients too much because that takes too much time.'

Nurses perceived great freedom in the counselling style they used, as this was hardly discussed with supervising physicians or colleagues. Conversations about patients were mostly about medical issues. Only a few nurses thought that this situation influenced the level of use of MI. Some nurses thought it would help to discuss counselling issues with colleagues. 


\section{Characteristics of nurses}

Although the nurses stated that they had enough knowledge and skills to implement MI, the structured part of the interview showed that they did not always interpret the MI strategies as intended. For example, one nurse said about 'agenda setting': 'Yes, I do tell patients what the purpose of the consultation is and how we're going to do it,' whereas 'agenda setting' has a different meaning in MI. Nurses did mention that they did not have a thorough command of MI and that it is not something they do automatically. According to the nurses, their own discipline and awareness are highly important for better use. 'I'm doing the consultations on my own all day and I feel I rapidly fall back into my own routine. I must remain constantly aware to keep on doing motivational counselling.' Some aspects, mainly basic principles and techniques, were in line with their own former work routines and with their prior nursing training (e.g. general health counselling). New skills were more difficult to learn than those acquired before, such as some basic principles and techniques. Skills that did not suit the nurses were often omitted. 'To me, exploring importance and confidence seems somewhat artificial. It doesn't suit me and therefore I simply don't use it.'

\section{Most important factors}

At the end of the interviews, nurses were asked to mention the three most important factors influencing the use of the method. The most frequently mentioned factors were: more training to reinforce the teaching material; more frequent feedback; the use of reminder cards; general practitioners should be informed about the techniques for more support; having enough time per patient; type of patient; and making conscious efforts to implement MI.

\section{DISCUSSION}

The current report describes an evaluation of an MI-based counselling training program for nurses in primary care. Despite approximately 21.5 hours of training, reminder cards and 5 hours of optional group meetings, the level of use was unsatisfactory. The skills that seem to have been improved by our training are agenda setting, assessing importance and confidence, summarizing, and avoiding unsolicited advice and/or information. Except the last one, these items were given special attention during the feedback visits and were also on 
the reminder cards. Accordingly, focusing on specific skills instead of the counselling style as a whole seems beneficial to the learning process.

The interviews showed that the nurses had a discrepant opinion about their level of use; they perceived themselves as performing better on all items. A possible explanation for this discrepancy could be that the taped consultations were not representative of diabetes consultations, as nurses selected 'checkup' patients to feel more confident during taped consultations, leaving little opportunity to implement MI. Patient influences on assessments of nurses' competence might have been reduced by using simulated patients playing the same role for every nurse (Kruijver, Kerkstra, Francke et al., 2000). However, we wanted to assess performance in everyday practice with 'real' patients, not in artificial circumstances with actors. Another explanation for the difference between observational and self-report scores could be that nurses are insufficiently aware of their own counselling behaviour. Nurses found it difficult to evaluate their own behaviour, as was reflected by their expressed need for more feedback. According to Social Comparison Theories (Festinger, 1954; Suls, Martin, \& Wheeler, 2002) people have a drive to evaluate themselves by comparing their performance with that of others. However, the only evaluation nurses have is based on patients' medical outcomes and patient satisfaction. While this evaluation is not necessarily related to the actual use of the method, satisfactory outcomes on these parameters might explain why nurses evaluated themselves differently than we did. A third possibility is that nurses gave socially desirable answers during the interviews. However, we continuously emphasized that our goal in the interviews was to learn from their experiences, rather than judging their competence. Also, most nurses remained positive about their use of the method after discussing the observational evaluations. This might reflect a self-serving bias; the observational evaluation would not reflect their actual use.

The interviews also showed that nurses thought this counselling strategy was not suitable for every consultation. It is not only the type of patient (e.g. immigrant patients with language problems) that matters, but also the time since diagnosis. Whereas lifestyle changes are often discussed shortly after the diagnosis, once a patient has stable and acceptable outcome parameters (HbAlc, blood pressure and lipid profile) lifestyle changes often have less priority and protocol items then become less relevant according to the nurses. In addition, general practice teams rarely discuss counselling styles or the way outcomes are achieved, by lifestyle changes or by medication. Also, nurses have to fill out patient data recording systems, which have a more anamnestic character, making it difficult to use the MI protocol. 
The Concerns-Based Adoption Model is a framework for understanding change in people (Horsley \& Horsley-Loucks, 1998). It distinguishes eight Levels of Use (LoU): non-use, orientation, preparation, mechanical, routine, refinement, integration, and renewal. Our training approach established a mechanical LoU instead of a routine LoU among nurses, reflecting early attempts to use new strategies, techniques, and materials, and a constant search for guidance and reassurance, as was apparent from some observations. First of all, nurses said they preferred implementing this style among 'easy' patients, whereas this style is specifically suitable for patients showing resistance. Nurses appeared not fully confident using MI. Second, nurses mentioned the risk of reverting to old routines when not making conscious efforts to use MI or when the perceived workload increases. Third, nurses argued that MI takes too much time and effort to use, while a few of them argued that this style yielded more structured consultations and a better understanding of patients, which can save time. Fourth, nurses explicitly indicated a need for more training (training sessions and feedback) and reminders. A need for more feedback reflects a need for more concrete and personalized training. Also, feedback sessions induce nurses to use $\mathrm{MI}$ in practice, which in turn yields more experience.

Our findings are in line with previous research about changing health professionals' behaviour. Two reviews addressing provider-patient communication describe the difficulties of changing a provider's behaviour. Van Dam and colleagues (2003) concluded that self-care and diabetes outcomes are more effectively improved by focusing on patient behaviour than provider behaviour, as the latter would be hard to change. Kruijver and colleagues (2000) found an effect on behavioural change among nurses in only two of 14 studies they reviewed. They cautiously suggest that trainers with a nursing background are more effective than trainers with a psychology background. Our intervention involved a nurse with much teaching experience who had attended MI training and constantly provided examples based on everyday practice. She also performed two of the three worksite visits. Nevertheless, the effect of our training program was relatively small. Miller and Mount (Miller \& Mount, 2001) described a 2-day clinical MI workshop for counsellors. Participants did increase some behaviours consistent with MI, but these were only added to prior counselling habits. These authors also found that the counsellors viewed themselves as fairly proficient, although, as in our study, this was unrelated to the quality of observed practices. Their counsellors perceived less need for further training, whereas our nurses perceived a great need for more training. This difference can be explained by our feedback visits, which stimulated self-reflection, although not to a sufficient extent, since 
nurses gave themselves more favourable scores than the research assistants did. Beneficial effects of feedback were also found in a study among dieticians in diabetes care (Brug et al., 2007), where most effects appeared not immediately after the training program but after personal feedback and advice. We therefore conclude that feedback on actions seems indispensable.

Our study had several limitations. First, the analysis of observational scores did not correct for multiple testing. However, correcting for multiple comparisons would have let us to conclude that on many items no statistical effect was observed, which would only have strengthened our conclusions. Second, we did not use a validated rating form because we only assessed the level of use of the skills we addressed in our training program. Our rating form may not have been sensitive enough to detect changes. Nevertheless, the results are in line with our experiences during feedback visits. Third, the results of the interviews about additional training activities were only based on experiences by the experimental group and not by the control group. We believe the differences in training program had negligible influence on the conclusions and implications, since both groups stressed the importance of feedback and more training sessions. Fourth, our use of audio-taped instead of video-taped consultations to assess the level of use precluded observation of non-verbal communication. However, the observations during feedback visits yielded largely the same impressions. Furthermore, video-taping might be even more threatening than audio-taping for nurses, which would have led to more unrealistic consultations.

\section{Conclusions}

Motivational Interviewing based counselling strategies are promising for diabetes care, but perhaps not suitable for every consultation and every patient. MI-based counselling may be specifically relevant for diabetes consultations in which self-management is the main topic to be discussed and expecting nurses to implement an MI approach during general diabetes care may be difficult. Practice characteristics such as paying attention to counselling methods need to be improved to facilitate the implementation of MI based strategies. The current project and previous studies indicate that changing health professionals' behaviour is a challenging process and requires sustained training efforts. Our training efforts of at least 21.5 hours should be extended with more training sessions and special attention should be paid to individual feedback and self- 
reflection. Further research is needed to optimize interventions to change counselling styles in everyday practice.

Our study has some implications for future training activities and research.

- The content of the training program should be reinforced in refresher sessions for a better recall of the meaning of specific training elements and general principles. Methods to do so should be developed.

- Instead of implementing a whole new counselling strategy, the current strategy could be divided into smaller parts (e.g. one week focusing on agenda setting only, another week on reflective listening etc).

- Counsellors should be reminded of the strategies and techniques during routine practice, for example by using reminder cards or adding reminders to patient data recording systems (e.g. a goal-setting field).

- Participants need continued feedback for more personalized and specific training. Feedback from colleagues in addition to feedback from trainers increases the feasibility of direct feedback and is beneficial for both the person being observed and the observer. Such contacts also provide peer support.

- Although nurses said that the use of MI depended largely on their own intrinsic motivation, extrinsic stimuli such as reminders and support by supervisors and colleagues would help.

- A more realistic perception of nurses' own actual behaviour requires selfreflective elements in the training program. An idea would be to ask nurses to score their own audio- or video-taped consultations.

- The use of this counselling strategy would be easier if patients with current lifestyle issues could make separate appointments in which lifestyle changes are the main topic of the consultation.

- Combining observational and self-report measures of practitioner behaviour is useful because they are complementary rather than overlapping.

- Direct observations during a random day in practice are necessary for a more realistic assessment of the level of use, as the nurses seemed to select patients for the audio-taped consultations, tending to avoid those for whom MI is especially useful, namely those having difficulties with behaviour change. 


\section{ACKNOWLEDGEMENTS}

We thank Moniek Lensen, Babs Fransen, Florence Hamers and Ineke Boertien from Maastricht University who scored audio-taped consultations. The study described was supported by a grant from the Dr. Paul Janssen Foundation.

\section{APPENDIX 5.1}

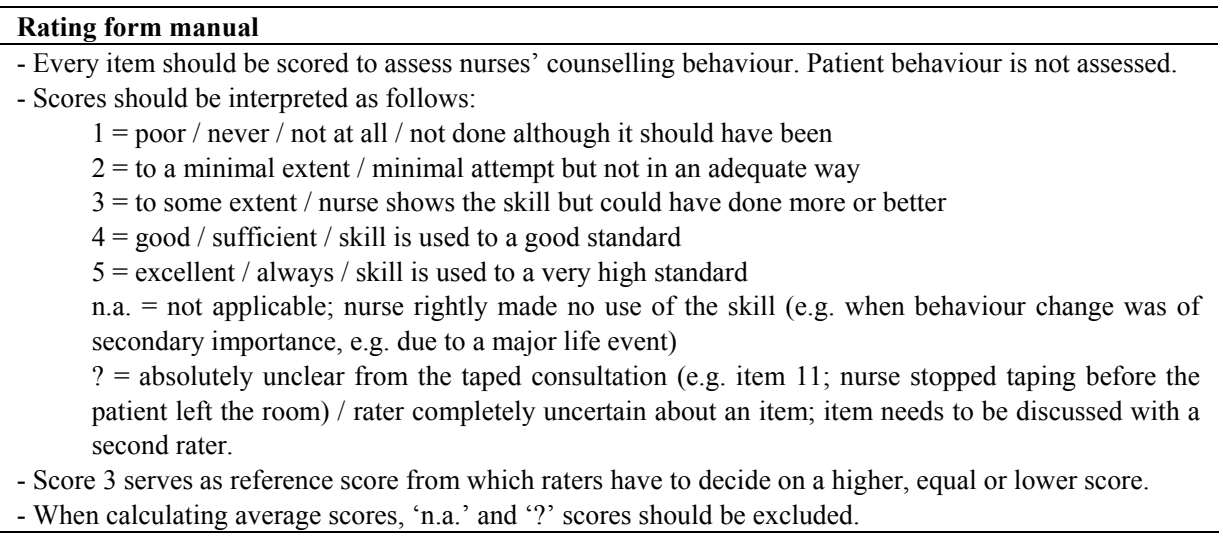


84 


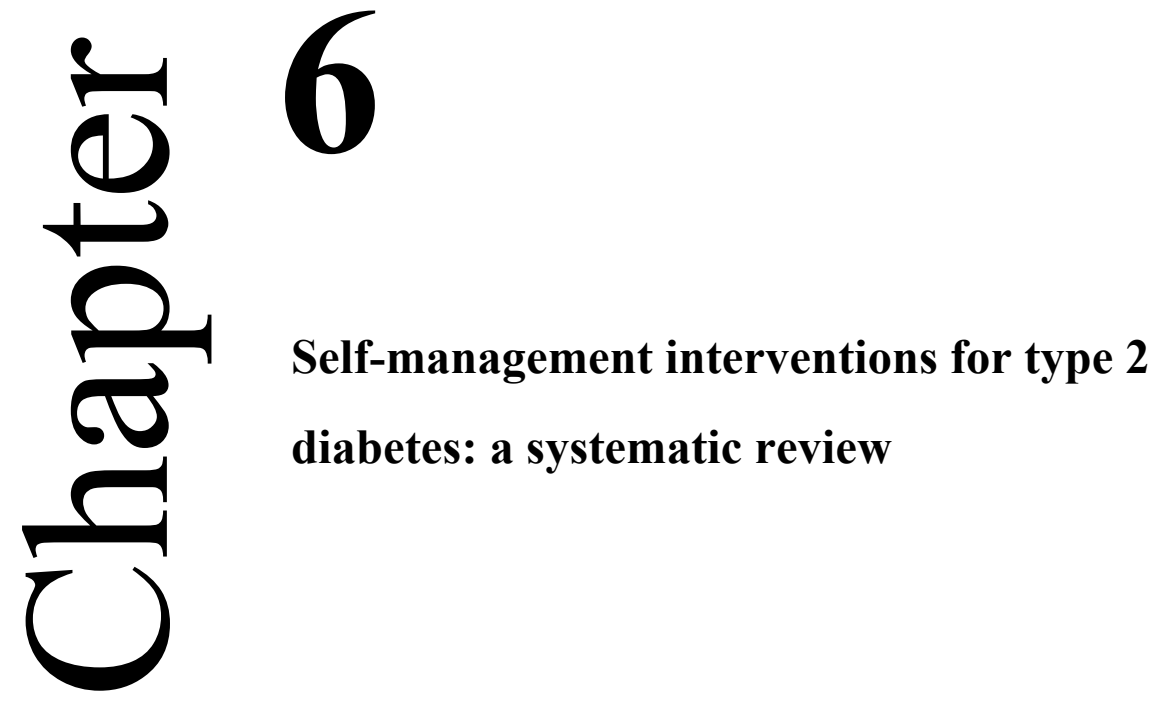

Published as: Heinrich, E., Schaper, N.C., \& De Vries, N.K. (2010). Self-management interventions for type 2 diabetes: a systematic review. European Diabetes Nursing, 7, 71-76. 


\begin{abstract}
This paper systematically reviews published randomised controlled trials, to determine the educational focus and effectiveness of type 2 diabetes multi-component selfmanagement interventions.

PubMed, PsycINFO,Web of Science and reference lists of included studies were searched for English-language articles published 2000-2010. Descriptive information was summarised; when possible, effect sizes were calculated.

Fourteen studies, described in 19 articles, were reviewed: six one-on-one interventions; six group interventions; two interventions comprising both intervention types. Four studies used learning as an intervention method; seven used learning and planning; three used learning, planning and practising. Self-management interventions seemed effective for diet, self monitoring of blood glucose, knowledge and diabetes specific quality-of-life (QoL); there were mixed results for exercise and clinical outcomes. Findings showed that dietary behaviour seemed relatively easy to change with self-management interventions. Group interventions with a practise component had the greatest potential to improve metabolic control. Self-management interventions had positive effects on diabetes-specific QoL and interventions using a collaborative learning approach improved knowledge.

Multi-component self-management interventions potentially lead to clinically relevant improvements in behaviour and some clinical parameters. Further research is needed to explain the mixed effects on exercise and to identify processes underlying behaviour change.
\end{abstract}




\section{INTRODUCTION}

Patients play a central role in diabetes care because of their daily responsibility for a large number of behavioural choices and activities. More concretely, patients have to take into account their diet, exercise, medication administration, blood glucose testing, smoking behaviour and medical examinations, including inspections of eyes and feet. Such self management is a complex activity and includes 'the ability to monitor one's condition and to affect the cognitive, behavioural and emotional responses necessary to maintain a satisfactory quality of life' (QoL) (Barlow et al., 2002). Self management is inescapable and it is a question of how, not whether, patients manage their diabetes. Although modern care and education are becoming more patientcentered (Funnell \& Anderson, 2004), many patients find it difficult to maintain adequate self management (Vermeire et al., 2005a). High-quality care is therefore needed, to better support patients' self-management abilities.

Reviews of articles published before 2000 show the potential benefits of self-management interventions but provide inconsistent results for various outcome measures (Brown, 1999; Norris et al., 2001; Norris et al., 2002). Numerous intervention studies have focused on improving one specific selfmanagement activity in people with type 2 diabetes, e.g. physical activity (PA) (Kirk, Mutrie, Macintyre et al., 2004) or dietary behaviour (Glasgow \& Toobert, 2000). However, to do justice to the complexity of daily practise, interventions are needed that focus on improving multiple self-management issues.

The objective of the present paper was to systematically review reports of randomised controlled trials (RCTs) to determine the methods and effectiveness of multi-component interventions aimed at self-management, in terms of changing behavioural, wellbeing, clinical and process outcomes for patients with type 2 diabetes (DM2). Interventions had to target at least two behaviours or had to be focused on self-management or diabetes in general.

\section{METHODS}

\section{Search methods}

Trials were identified by searching PubMed, PsycINFO and Web of Science. Four key-word clusters were used; diabetes (DM2), health behaviour (e.g. lifestyle), interpersonal relations (e.g. patient participation), and education (e.g. 
patient education). Figure 6.1 illustrates the electronic search and selection processes used. In addition to this search, reference lists of included studies and reviews were scanned manually for relevant publications. The search was undertaken in March 2010 and was restricted to articles published in English between January 2000 and March 2010.

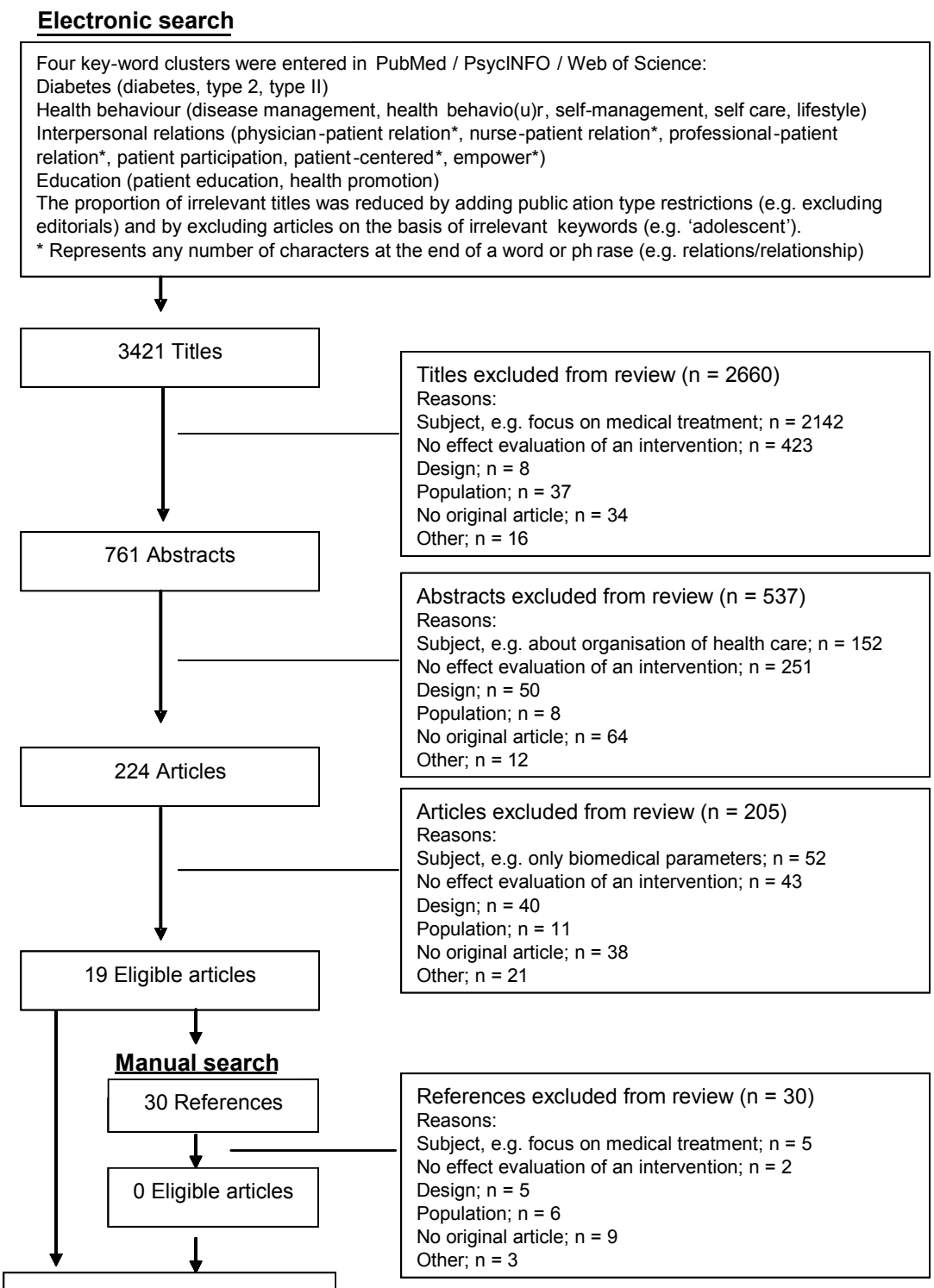

Total: 19 Included articles

Figure 6.1 Study selection process for the search involving studies published between January 2000 March 2010 


\section{Study selection}

Studies were included if they described the outcomes of an effect evaluation of a diabetes self-management intervention; the population consisted of adults with type 2 diabetes (aged $\geq 18$ years); the trial was a RCT with a pre-test / post-test design.

Studies were excluded if the intervention only focused on one selfmanagement component; clinical parameters were the only published outcome measures (because self-management interventions are targeted at changing behaviours, which does not necessarily result in clinical improvements); the study did not describe specific results for type 2 diabetes; the control group received an alternative intervention (because these interventions might vary widely).

Specific reasons for exclusion were coded and categorised into: inappropriate subject (e.g. medical treatment), goal of the paper (e.g. protocol article), research design (e.g. single-group design), population and article category (e.g. letter to the editor), see Figure 6.1. The selection started with an assessment of titles and for all relevant titles, abstracts were assessed. Full articles were retrieved if abstracts were found to be relevant, when there was no abstract or whenever there was any doubt about the relevance.

\section{Intervention categorisation and outcome classification}

Interventions were categorised as group versus one-on-one. Outcomes were classified as behaviour, wellbeing, clinical outcomes, and process outcomes. For every significant intervention effect, the magnitude of the effect size (ES) (Cohen's $d$ ) was calculated whenever possible (Cohen, 1988). Effect sizes were defined as small ( $\mathrm{ES} \leq 0.32$ ), medium (ES 0.33-0.55), and large ( $\mathrm{ES} \geq 0.56$ ). These interpretations were based on Lipsey's guidelines for the specific domain of treatment effectiveness research in the behavioural sciences (Lipsey, 1990).

\section{RESULTS}

From the 3421 articles that were generated (Figure 6.1), 19 were retained for this review; these described 14 independent studies. Study characteristics, the content of interventions and the reported outcomes are summarised in Table 6.1. The articles are arranged in order of intervention type (one-on-one versus group). 


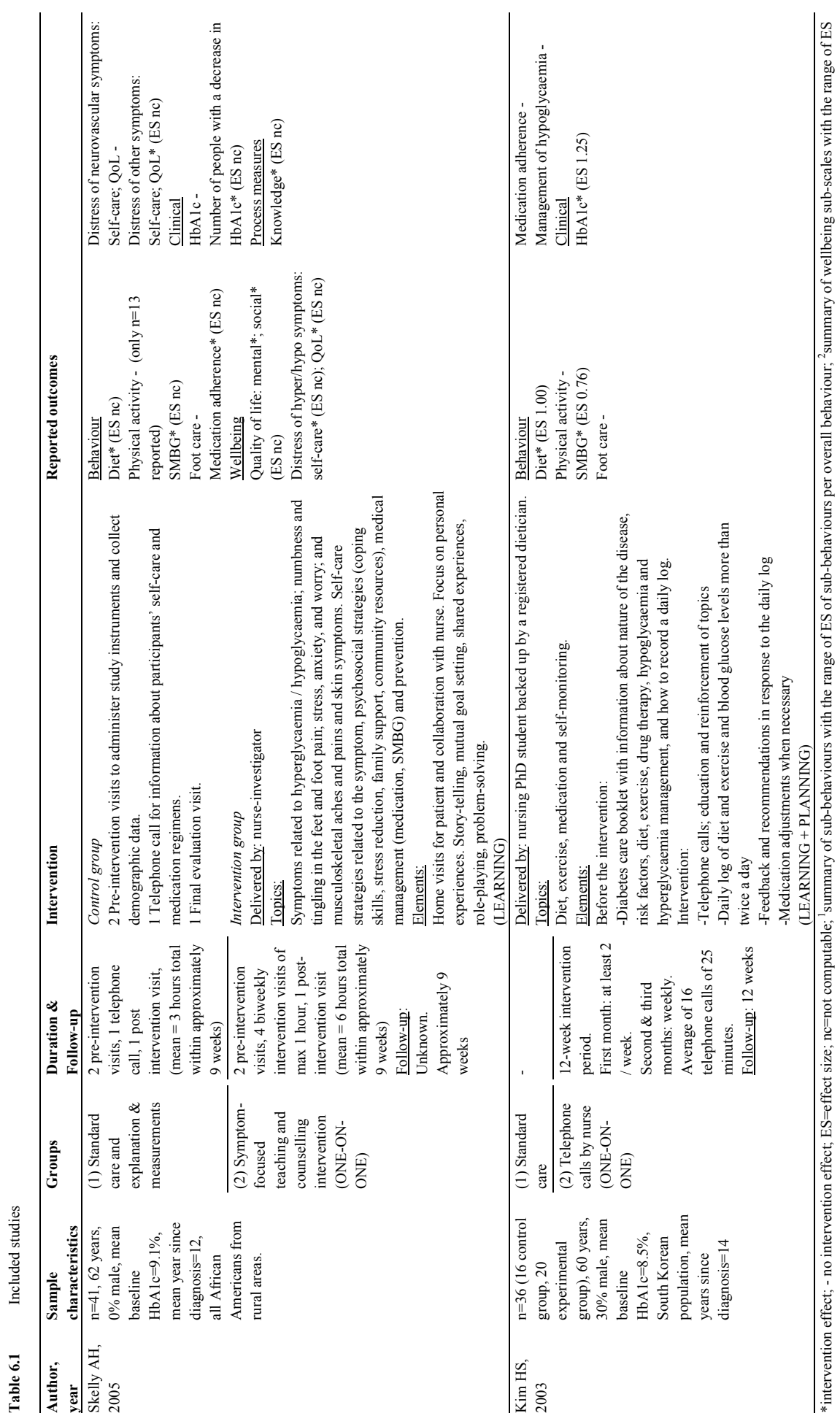




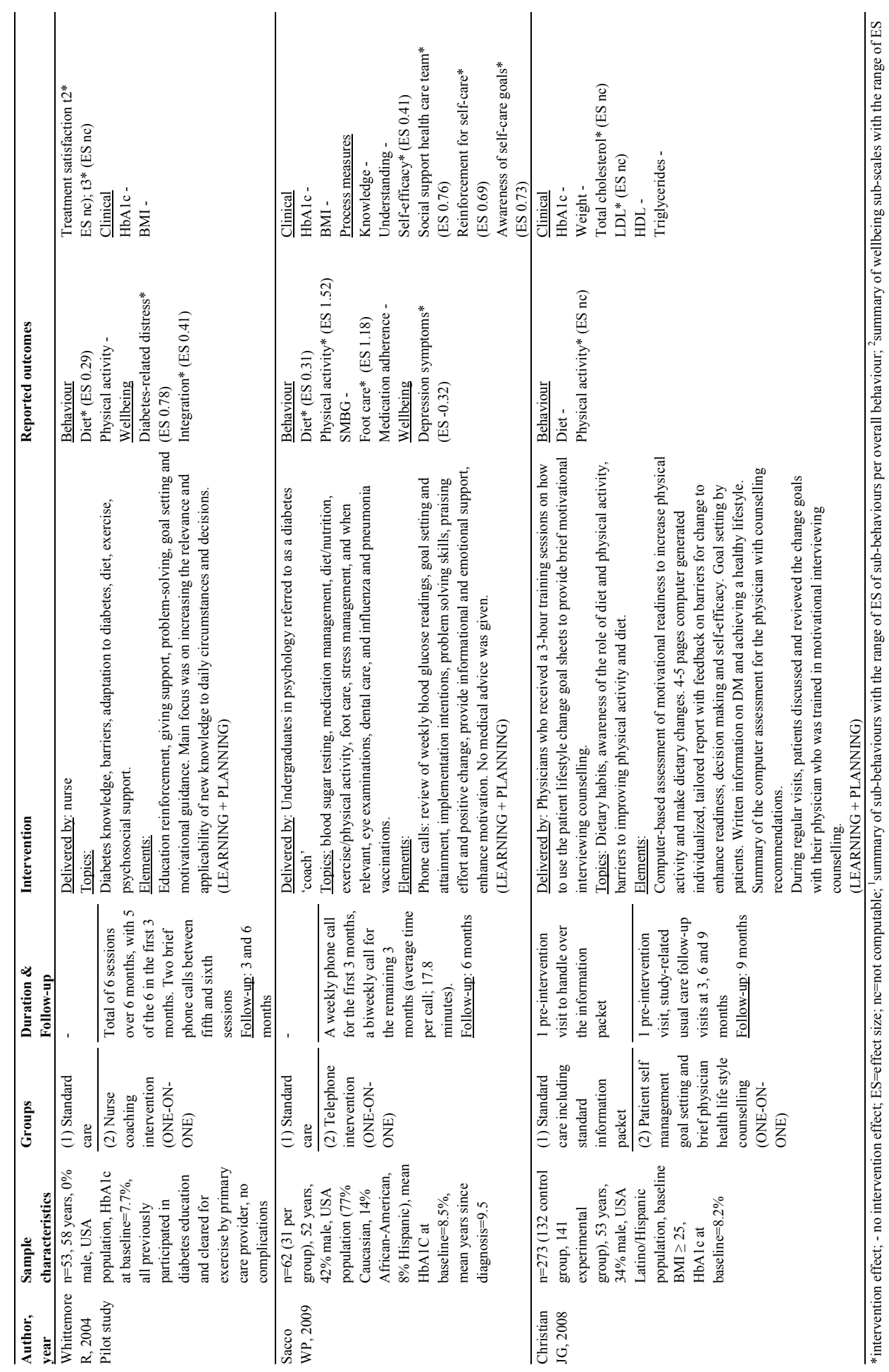




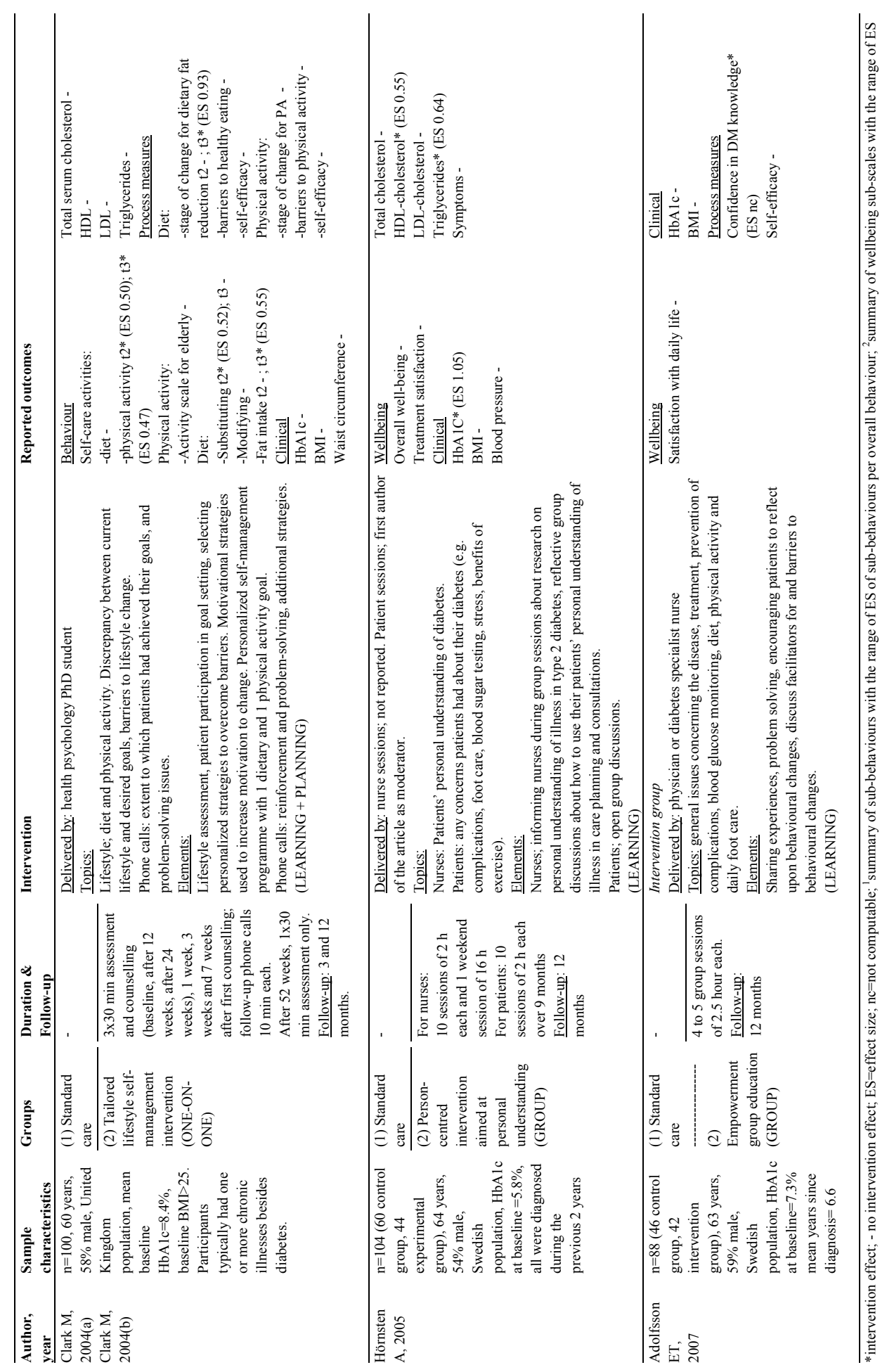




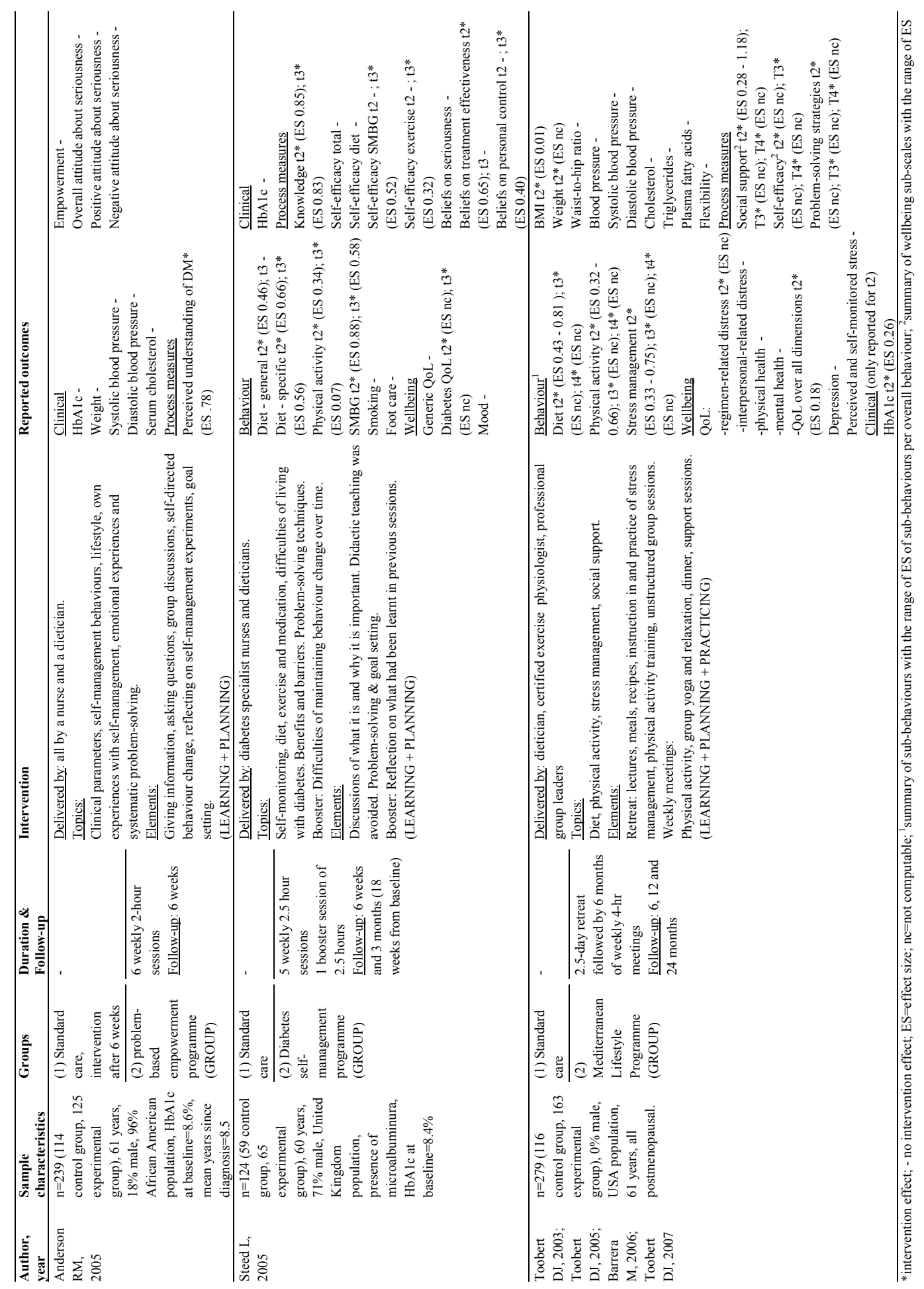




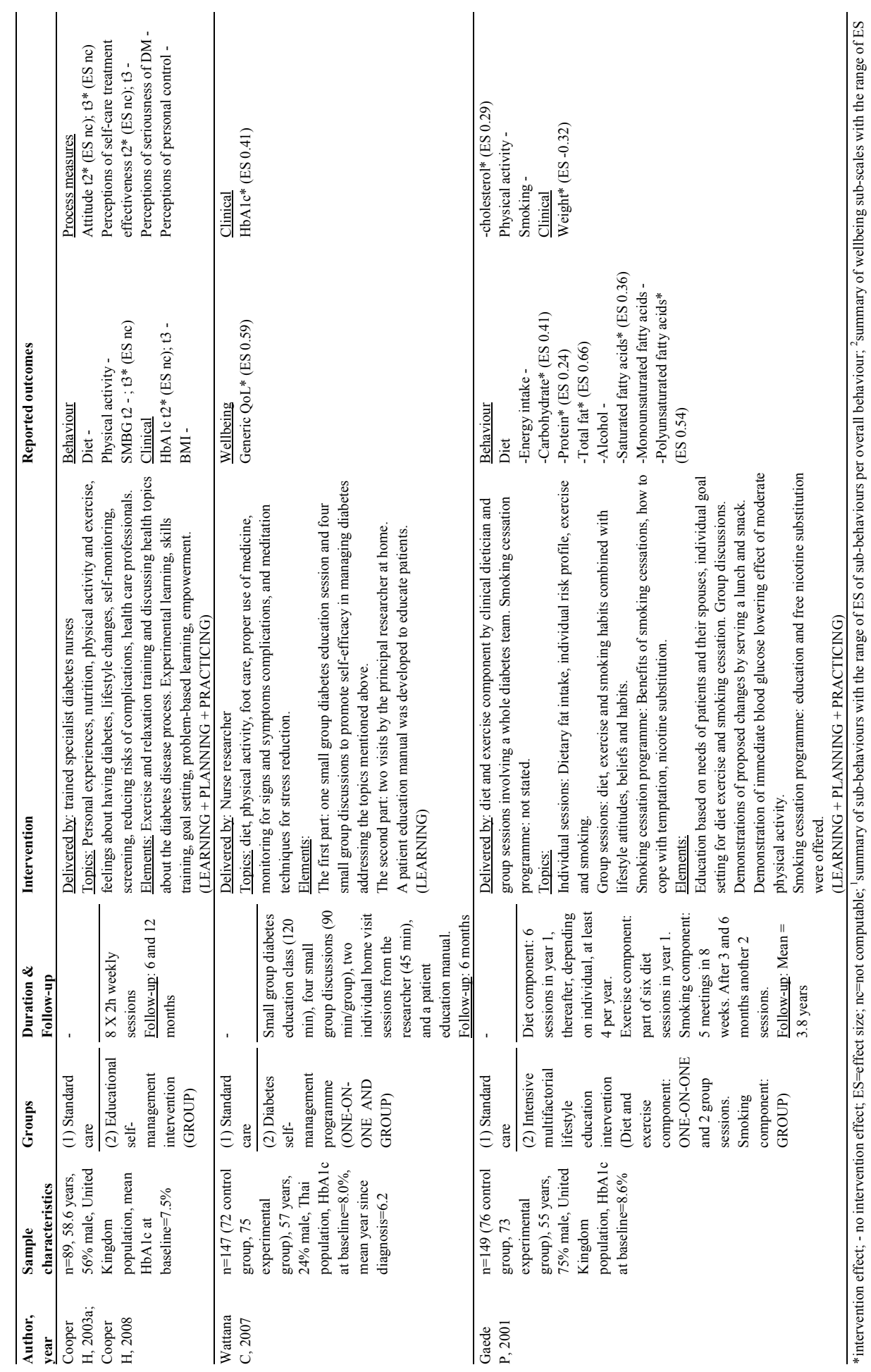




\section{Study characteristics}

Sample characteristics differed across the studies, which were carried out in North America $(n=6)$, the United Kingdom $(n=4)$, Sweden $(n=2)$, South Korea $(n=1)$ and Thailand $(n=1)$; see Table 6.1. Three of the American studies had specific target populations: African-American adults (Anderson, Funnell, Nwankwo et al., 2005; Skelly, Carlson, Leeman et al., 2005) and Latino/Hispanic adults, who also had to meet additional criteria (Christian, Bessesen, Byers et al., 2008); three studies only included women (Skelly et al., 2005; Toobert, Glasgow, Strycker et al., 2003; Whittemore, Melkus, Sullivan et al., 2004). Sample sizes ranged from 36 (Kim \& Oh, 2003) to 279 (Toobert et al., 2003) (mean=127).

Eleven studies described informed-consent procedures (written informed consent $n=9$; verbal informed consent $n=2$ ). Three studies did not mention informed consent, but two described ethics committee approval.

In all studies, the control group received their usual care. If the control group received diabetes information, this was restricted to information that would normally be imparted during usual care.

Six studies described one-on-one interventions (Christian et al., 2008; Clark et al., 2004a; Kim \& Oh, 2003; Sacco, Malone, Morrison et al., 2009; Skelly et al., 2005; Whittemore et al., 2004), another six evaluated group interventions (Adolfsson, Walker Engstrom, Smide et al., 2007; Anderson et al., 2005; Cooper, Booth, \& Gill, 2003a; Hornsten, Lundman, Stenlund et al., 2005; Steed, Lankester, Barnard et al., 2005; Toobert, Strycker, Glasgow et al., 2005), and two combined both intervention types (Gaede, Beck, Vedel et al., 2001; Wattana, Srisuphan, Pothiban et al., 2007). In addition, there was a large variation in intervention intensity. For example, Christian and colleagues (2008) described an intervention in which patients only had a pre-intervention visit in addition to usual care, where a computer-based lifestyle assessment took place. Toobert and colleagues (2003) described the most intensive intervention, which consisted of a 2.5 day retreat followed by 6 months of weekly 4-hour meetings. Follow-up duration ranged from 6 weeks (Anderson et al., 2005) to 3.8 years (Gaede et al., 2001).

In two of the studies, patients' usual health care providers were involved (Christian et al., 2008; Hornsten et al., 2005); in four studies, the interventionist was a PhD student or researcher (Clark et al., 2004a; Hornsten et al., 2005; Kim \& Oh, 2003; Sacco et al., 2009) and in other studies health care professionals (e.g. nurses and dieticians) implemented the intervention. The intervention described by Christian and colleagues (2008) took place during 
usual care, after the computer-based lifestyle and motivational assessment. In all other studies, the intervention activities were additional to usual care.

The studies included in our review described different intervention methods which could be categorised into three main types: 'learning' (information / education), 'planning' (create a self-management plan) and 'practising' (practise self-management behaviours). Four interventions consisted of learning only (Adolfsson et al., 2007; Hornsten et al., 2005; Skelly et al., 2005; Wattana et al., 2007), seven included learning and planning (Anderson et al., 2005; Christian et al., 2008; Clark et al., 2004a; Kim \& Oh, 2003; Sacco et al., 2009; Steed et al., 2005; Whittemore et al., 2004), and three used all three methods (Cooper et al., 2003a; Gaede et al., 2001; Toobert et al., 2003).

Seven studies described the theoretical background of their intervention (Adolfsson et al., 2007; Anderson et al., 2005; Christian et al., 2008; Clark et al., 2004a; Cooper et al., 2003a; Gaede et al., 2001; Hornsten et al., 2005; Kim $\&$ Oh, 2003; Sacco et al., 2009; Skelly et al., 2005; Steed et al., 2005; Toobert et al., 2003; Toobert, Glasgow, Strycker et al., 2007; Wattana et al., 2007; Whittemore et al., 2004), four described some theoretical concepts (e.g. selfefficacy) without referring to specific theories (Adolfsson et al., 2007; Anderson et al., 2005; Christian et al., 2008; Clark et al., 2004a; Cooper et al., 2003a; Gaede et al., 2001; Hornsten et al., 2005; Kim \& Oh, 2003; Sacco et al., 2009; Skelly et al., 2005; Steed et al., 2005; Toobert et al., 2007; Wattana et al., 2007; Whittemore et al., 2004), and three described no theoretical foundation (Adolfsson et al., 2007; Anderson et al., 2005; Cooper et al., 2003a; Hornsten et al., 2005; Kim \& Oh, 2003; Sacco et al., 2009; Skelly et al., 2005; Steed et al., 2005; Toobert et al., 2007; Wattana et al., 2007; Whittemore et al., 2004).

Although all studies addressed self-management, the focus of the interventions varied (Table 6.1). Six studies covered a broad range of diabetesrelated topics including self management (Adolfsson et al., 2007; Cooper et al., 2003a; Hornsten et al., 2005; Skelly et al., 2005; Wattana et al., 2007; Whittemore et al., 2004), five had a narrower focus on self-management behaviours (Anderson et al., 2005; Kim \& Oh, 2003; Sacco et al., 2009; Steed et al., 2005; Toobert et al., 2007), and three covered very specifically targeted lifestyle changes (Christian et al., 2008; Clark et al., 2004a; Gaede et al., 2001).

\section{Outcomes}

Ten studies reported behavioural outcomes, of which diet and PA were by far the most reported. An intervention effect on diet was observed in eight of the 10 studies, with ES ranging from 0.29 (Whittemore et al., 2004) to 1.00 (Kim 
$\&$ Oh, 2003). Positive effects were found with different intervention types (in both one-on-one and group interventions). Moreover, effects were seen in different studies, regardless of the method (learning/planning/practising) used. One relatively large one-on-one intervention, specifically focused on changing lifestyle, did not find any effects (Christian et al., 2008). In contrast to other studies, this intervention mainly took place during usual care consultations with one additional pre-intervention lifestyle assessment.

Concerning PA, five of the 10 studies found positive effects on PA, mostly reporting medium to large ES. A relatively small study found the largest effects (Sacco et al., 2009). Effective studies did not differ from ineffective studies with respect to intervention type or method, but effective studies focused primarily on self-management behaviours (Sacco et al., 2009; Steed et al., 2005; Toobert et al., 2007) and lifestyle changes (Christian et al., 2008; Clark et al., 2004a). Three of the five ineffective studies focused on a variety of diabetes-related topics (Cooper et al., 2003a; Skelly et al., 2005; Whittemore et al., 2004). Furthermore, in the sample sizes of effective studies were usually higher compared with the sample sizes of the ineffective studies, except for the study by Sacco and colleagues (2009). As in studies with dietary outcomes, four of the five studies measuring the frequency of self monitoring of blood glucose (SMBG) found positive effects with large ES, regardless of intervention type and method. A limited number of studies $(n=4)$ evaluated foot care. Again, the intervention described by Sacco and colleagues (2009) was the only effective study, with a large ES (1.18).

Five of thirteen studies observed lower glycosylated haemoglobin (HbA1c) levels in the intervention group compared with the control group at postmeasurement, with ES ranging from 0.26 to 1.25 (Cooper et al., 2003a; Hornsten et al., 2005; Kim \& Oh, 2003; Toobert et al., 2003; Wattana et al., 2007). Four of the five effective studies included group sessions. Regarding intervention methods, learning and planning strategies were used in both effective and ineffective studies. Both of the studies where the intervention included 'practising' showed lower HbA1c levels in the study group compared with the control group (Cooper et al., 2003a; Hornsten et al., 2005; Kim \& Oh, 2003; Toobert et al., 2003; Wattana et al., 2007). Body mass index (BMI) / weight was the second most-reported clinical outcome. One of the 10 studies, which evaluated a group intervention with learning, planning and practising methods, found a lower BMI in the intervention group compared with the control group (Toobert et al., 2003), with a small ES (0.01). Three studies explicitly focused on lifestyle changes (Christian et al., 2008; Clark et al., 2004a; Gaede et al., 2001), of which one showed effects of diet and PA, but 
weight did not change (Clark et al., 2004a). Other clinical outcomes, such as blood pressure and lipid profiles, were less frequently reported. No effects were found on blood pressure but two studies, which used different intervention methods, found positive effects on lipid profiles. Clark and colleagues (2004a) and Toobert and colleagues (2003) showed positive effects of diet and PA; however these behavioural changes did not alter the biomedical measures.

Beneficial effects of interventions were shown in all four of the studies that measured diabetes-specific quality-of-life (QoL) (Skelly et al., 2005; Steed et al., 2005; Toobert et al., 2003; Whittemore et al., 2004). In addition, two out of the four interventions resulted in better generic QoL in patients compared with those in the control group (Skelly et al., 2005; Wattana et al., 2007). Interventions that improved QoL varied in type and method. Concerning the two most frequently measured process outcomes, four of the five studies assessing knowledge or perceived understanding of diabetes (Adolfsson et al., 2007; Anderson et al., 2005; Skelly et al., 2005; Steed et al., 2005), and three of the five studies assessing self-efficacy (Sacco et al., 2009; Steed et al., 2005; Toobert et al., 2007) reported positive effects of the intervention. Effective interventions varied in intervention type and method, but all three group interventions resulted in better knowledge levels in treated patients compared with controls (Adolfsson et al., 2007; Anderson et al., 2005; Skelly et al., 2005; Steed et al., 2005).

\section{DISCUSSION}

This review sought to determine the methods and effectiveness of multicomponent interventions aimed at self-management for type 2 diabetes. Concerning behavioural outcomes, diet and exercise were frequently measured. The effects of self-management interventions are highly promising for making dietary changes. In contrast, the benefits of self-management interventions for exercise are more ambiguous, although interventions with a specific focus on behaviour change show potential, and the relative lack of efficacy may have been caused by limited power. Differences in effectiveness could not be attributed to other intervention or study characteristics. Similar observations were made by Norris and colleagues (2001) in their review of studies published before 2000; they were unable to establish any factor that could explain variations in effectiveness.

No conclusions could be drawn for SMBG or foot care because only a limited number of interventions have addressed these outcomes. However, 
interventions with SMBG as a specific topic seemed successful in improving the frequency of monitoring, regardless of intervention characteristics.

Evidence for the effectiveness of interventions on clinical outcomes was less conclusive. Group interventions with a practise component seemed to be most promising for improving metabolic control; these results are supported by earlier reviews demonstrating the potential benefits of group interventions for optimizing HbA1c (Deakin et al., 2005; Duke, Colagiuri, \& Colagiuri, 2009). However, inconsistent results were found for lipid profiles, and no effect was found for blood pressure. This lack of effect on blood pressure is in contrast with the Cochrane review of 2005 (Deakin et al., 2005), in which pooled analyses revealed that group-based self-management programmes resulted in blood pressure reductions. Given the heterogeneity of the various studies, it was impossible to pool effects across studies. No conclusions could be made about BMI/weight outcomes since only one of the 10 studies reporting on these parameters showed any improvements (Toobert et al., 2003).

Wellbeing and process measures varied widely and not all studies reported results for these outcome categories. Most evidence was found for the effect of self-management interventions on disease specific QoL and knowledge: disease-specific QoL instruments are known to be more sensitive to change than generic instruments (Guyatt, Feeny, \& Patrick, 1993). The outcomes for knowledge are also in line with an earlier review by Brown and colleagues (1999).

In general, the various interventions seemed more effective in improving behavioural outcomes than clinical parameters. Newman and colleagues (2004) argued that researchers often incorrectly assume a simple relation between behaviour change and clinical state, because clinical parameters are usually influenced by many more factors than a specific altered behaviour (Newman, Steed, \& Mulligan, 2004). Moreover, behavioural outcomes were mostly measured by self-reporting questionnaires, which are liable to bias (Bartholomew et al., 2000). Additional explanations for the apparent lack of efficacy on clinical parameters could be that most interventions did not specifically address behaviour change, and the observation period used in several studies may have been too short.

Studies included in the current review varied markedly in intervention method. Concerning learning as method, only one intervention used a somewhat didactic method (Wattana et al., 2007), whereas the review by Norris and colleagues included eight such interventions (Norris et al., 2001). The more collaborative approaches that we found are in line with the shift within 
educational interventions from didactic teaching approaches towards more patient-centered or 'empowerment' approaches (Glasgow \& Anderson, 1999).

Comparing intervention studies on self-management could be facilitated by standardized intervention descriptions (Abraham \& Michie, 2008; Davidson, Goldstein, Kaplan et al., 2003) and measures of behaviour change (Semaan, Des Jarlais, Sogolow et al., 2002), and better descriptions of the translation of theoretical concepts to the interventions (Michie \& Abraham, 2004). Furthermore, future research should report process outcomes, and interactions between different outcomes, to obtain a better understanding of the underlying processes of change (Clark et al., 2004b; Cooper et al., 2003a; Cooper, Booth, $\&$ Gill, 2003b; Norris et al., 2001).

The present review had several limitations. In almost all studies reviewed, the intervention was offered on top of usual care. Extra contact time for patients in the experimental groups may have led to an overestimation of effectiveness (Norris et al., 2002). Also, the quality of each study has not been assessed and rated. Furthermore, only RCTs were included, and only those published between 2000 and 2010.

In conclusion, multi-component self-management interventions are effective in changing dietary behaviour - independent of type and method - and diabetes-specific QoL; group interventions with a practise component have the potential to improve metabolic control; and interventions using a collaborative learning approach can improve understanding of diabetes and some selfmanagement behaviours. The fact that most interventions had medium-to-large ES on outcomes suggests that self-management interventions can potentially lead to clinically relevant improvements in behaviour and also in some clinical parameters. Since there is no difference in effectiveness between the two intervention types for behavioural outcomes (one-on-one versus group), group interventions are preferable as they are often more cost-effective. Further research is necessary to explain the inconsistent effects of self-management interventions on PA, and to investigate the processes underlying behaviour change. Finally, we suggest that in future studies investigating the effect of self-management education, the intervention should be embedded in daily care. This would enable realistic comparisons to be made between the control and intervention groups, and would facilitate the implementation of promising new strategies. 


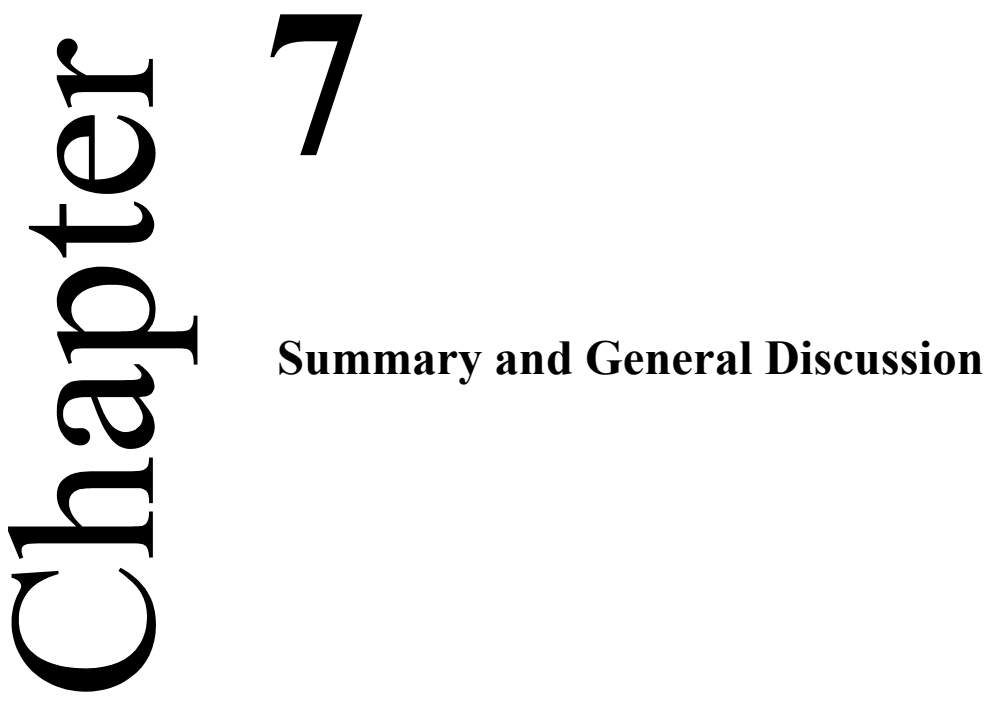




\begin{abstract}
In this final chapter, an overview of the research described in this dissertation is given followed by the general discussion. The chapter starts with a short introduction of the problem, a description of the current project, and a summary of the studies including the main outcomes. In the second part of this chapter, developments in chronic care are addressed and our project is discussed from a practical as well as methodological perspective. Finally, recommendations for future research and practical implications are given.
\end{abstract}




\section{INTRODUCTION}

Patients with type 2 diabetes mellitus (DM2) experience difficulties with adequate self-management (Bonnet et al., 2001; Harris, 2000), resulting in suboptimal outcomes (Funnell, 2006; Nelson et al., 2002) with an increased risk of complications and a decreased life expectancy and quality of life (Funnell \& Anderson, 2004; Resnick et al., 2006). Self-management is not only challenging for patients; also health care professionals have problems with finding effective ways to optimally support patients with diabetes (Larme \& Pugh, 1998). During the last two decades the organization and the content of diabetes care have changed from the more paternalistic medical care model towards more patient-centered care. Effective self-management interventions that fit these changes are needed, especially since the incidence of type 2 diabetes still increases (International Diabetes Federation, 2010).

\section{The current project}

The main aim of the research described in this dissertation was to develop and evaluate an evidence-based education and counselling strategy to support selfmanagement of DM2 patients and to provide recommendations on how to optimize patients' self-management. As described in the chronic care model (Wagner et al., 2001; Wagner et al., 2005), patient outcomes are a result of productive interactions between informed, activated patients on the one hand and a prepared, proactive team of health care professionals on the other. Interventions solely targeted at patients would ignore the importance of this relationship. Also, successful approaches are often multifaceted (Renders, Valk, Griffin et al., 2001). Therefore, we decided to develop an intervention directed at both, patients and health care professionals and our strategy consisted of two components: a web-based education programme and a Motivational Interviewing based counselling training for practice nurses.

The changes in diabetes care, as described in the general introduction of this dissertation, may have led to changes in self-management interventions. Therefore, we reviewed recently published reports on type 2 diabetes selfmanagement interventions to determine the educational focus and effectiveness of these interventions. 


\section{SUMMARY OF THE STUDIES}

\section{www.diep.info}

Using the steps of a framework for decision makers of programme development (Intervention Mapping) (Bartholomew et al., 2000) and in cooperation with all representatives of relevant diabetes (DM) organizations, the first component of the diabetes education and counselling strategy was developed; the web-based education programme (DIEP; Diabetes Interactive Education Programme). We started with a needs assessment consisting of a literature review and focus-group interviews among potential programme users to analyze the target group and its problems and to define the most important needs with regard to diabetes education and counselling. Factors were identified which are associated with improving self-management such as informed decision making (Funnell \& Anderson, 2002) and goal setting (Van Dam et al., 2003; Wens et al., 2008). During the interviews, health care professionals argued that many patients have insufficient understanding of their disease in order to participate as equal conversation partner, to share responsibility and to formulate goals. Patients however, felt little need for further education but kept on asking the interviewers questions about diabetes. Also, none of the patients reported that they had ever been asked what they wanted, reflecting an unequal partnership in decision making. The assessment illustrated the need for a patient education programme which can improve knowledge, stimulate self-management and supports patient participation in DM care.

The step-wise developmental process, as described in Chapter 2, resulted in www.diep.info; a unique education programme consisting of seven chapters with basic and additional information, a dictionary, self-management checklists and a workbook for goal setting and preparation for consultations. The information included is mostly spoken text supported by headlines, images, video and patient experiences. The programme aims to increase patients' understanding of DM2, to support communication between health care professionals and patients, to stimulate active patient participation and to provide tools to support adequate self-management.

Both an effect evaluation and a user evaluation were performed which are more detailed described in Chapter 3. The effect evaluation $(n=99)$ showed the ability of the website to increase patients' understanding of DM2. The user evaluation, consisting of an online survey $(n=564)$ and interviews $(n=11)$, showed that although the programme received mainly positive evaluations, the 
programme was merely used as an information tool instead of a tool to support communication, active patient participation and adequate self-management.

Patients with a chronic condition need a sufficient understanding of the disease to be able to make informed choices concerning health related behaviour and to implement self-care plans with individual goals (Puder \& Keller, 2003). By increasing DM knowledge, the programme www.DIEP.info can encourage self-management; not through a direct effect on behaviour because the programme is not used as intended, but as a facilitator and stimulator. Currently, nurses make patients aware of the existence of our website and thereby our programme is solely used as source of information for patients. More efforts are needed to stimulate ongoing education with a more optimal use so that the programme can contribute to factors that have been shown to improve self-management such as active patient participation (Funnell \& Anderson, 2002; Rollnick et al., 1999; Van Dam et al., 2003; Williams et al., 2005) and mutual goal setting (Van Dam et al., 2003; Wens et al., 2008) by using the workbook and the goal setting form. An enhanced use of the programme requires a more active role of health care professionals in the implementation of the programme. A lack of instructions to health care professionals that participated in the education programme studies is the most likely explanation for the suboptimal use and therefore it is likely that the effects of the programme will be extended when professionals are trained in using the programme during DM consultations. As a result of our project a 12hour certified course called diep@work has already been developed to train nurses in the use of our education programme during daily practice. The training consists of 2 group sessions, homework, an action plan, reminders and a follow-up visit. The implementation of diep@work has already started.

\section{MI-based counselling training}

The second and complementary component of our education and counselling strategy was based on the basic principles of Motivational Interviewing (MI) (Miller \& Rollnick, 2002) and health behaviour change counselling (Rollnick et al., 1999). This component comprised a training for practice nurses in primary diabetes care, focussing on their health counselling skills to facilitate patient support with adequate self-management. We targeted at practice nurses who specialize in chronic care because they are responsible for the daily care for patients with type 2 diabetes, under the supervision of a general practitioner. A basic group training course was given, followed by individual feedback (written and personal) and follow-up group meetings. The total 
training duration was 21.5 hours and 5 hours of optional group meetings. During the two training course days, basic principles and techniques of motivational interviewing were addressed as well as an MI consultation protocol. Additionally, during this MI-based counselling training, nurses were introduced to the education programme and an explanation was given about how to use this programme in educating patients. Special attention was paid to the link between the skills that were learned and helpful tools in the education programme. For example, one of the tasks described in the MI-based protocol is 'setting concrete, realistic goals with a patient' and one of the selfmanagement support tools in the programme is a goal setting form on which the current status and desired goals can be written down. After being instructed, nurses could introduce the programme to patients so that patients could use it at home and prepare themselves for the next consultation for example by writing down questions about the information they have encountered on the website. The first individual feedback for nurses consisted of a letter with feedback to audio-taped consultations which were taped 3 months after the group training. Based on these consultations, it was decided to provide more individual implementation support. Approximately 6,9 and 11 months after the group training, nurses received immediate personal feedback during practice visits.

The training given to nurses was evaluated in a cluster randomized controlled trial with a follow-up time of two years. Thirty-three nurses and 584 patients participated. Both an effect evaluation and an implementation evaluation were carried out and are described in Chapter 4 and Chapter 5 of the current dissertation. No structural use of our education programme was observed among patients and therefore, the effect evaluation was focused at the MI-based counselling strategy; clinical, behavioural and process outcomes were assessed. The results indicated no major beneficial effect of the training, except for one health locus of control dimension and knowledge. Adverse effects were found for fat intake and HDL-cholesterol. On the basis of this study it cannot be concluded that the MI-based counselling training yielded advantageous outcomes over usual care by untrained nurses. The implementation study assessed the effects of the training on the level of use of the MI method in diabetes consultations, and explored factors influencing the use of the MI method. Concerning the level of use, only five out of twenty skills that were scored were positively affected by our training, which was a rather modest effect. Four of these five items were given special attention during the feedback visits. Nurses however, perceived themselves as performing better on all items. The most important implementation factors influencing the level of use were (according to the nurses): the amount of 
training and feedback, reminders, perceived workload, time per patient, type of patient, and support from supervisors and colleagues. This implementation study provided the opportunity to put the outcomes of the effect evaluation into perspective. The most likely explanation for the limited effect of the training on patients' outcomes is the level of implementation of MI-based counselling skills. Future studies should pay specific attention to training strategies and intervention implementation in usual care.

\section{Review}

Diabetes care has changed over the past years and this may have led to changes in research targeted at self-management interventions. As described in Chapter 6, a review of the most recently (2000-2010) published reports was carried out, to determine the educational focus and the effectiveness of self-management interventions for DM2 patients. This review was specifically aimed at interventions that target at least two diabetes relevant behaviours or that are focused on self-management or diabetes in general, because self-management is complex as it comprises more than one single behaviour. Self-management is not only about changing unhealthy habits into a healthy lifestyle, it is also about adapting behaviour to one's constantly changing condition and specific circumstances.

In total, 14 studies were reviewed which led to the conclusion that selfmanagement interventions are clearly beneficial for dietary behaviour, diabetes specific quality of life and knowledge. Mixed results were found for exercise and clinical outcomes, and very few interventions were able to reduce weight. Intervention type (one-on-one versus group) and methods (learning / planning / practising) did not make a clear difference in effectiveness, although group interventions with a practise component seemed to have the highest potential to improve metabolic control. Wellbeing and process measures varied widely and not all studies reported measures of these outcomes which made it difficult to draw conclusions about the underlying mechanisms of the different interventions.

The interventions that were evaluated in studies in this review mostly took place in addition to usual care visits, for example separate diet, physical activity and smoking group sessions (Gaede et al., 2001). In only two studies usual health care providers were involved as interventionists (Christian et al., 2008; Hornsten et al., 2005) and in only one of these studies the intervention mainly took part during usual care consultations. Another characteristic of the included studies was that didactic teaching methods were hardly applied 
whereas the review by Norris et al. of studies before the year 2000 included eight such interventions (Norris et al., 2001). So, research targeted at selfmanagement interventions indeed seemed to have changed in line with changes in diabetes care.

\section{DISCUSSION}

\section{Developments in chronic care}

In the Netherlands, the number of people suffering from a chronic disease such as diabetes mellitus and chronic obstructive pulmonary disease (COPD) increases rapidly (Baan \& Poos, 2009; Boezen, Postma, Smit et al., 2006). This can be attributed to the fact that the Dutch society is ageing (De Hollander et al., 2006), but also to the increased presence of risk factors for chronic diseases, such as lifestyle factors (Stampfer, Hu, Manson et al., 2000; WHO, 2009). The growing number of patients with a chronic disease requires not only more but also a different kind of health care.

Originally, health care was mainly a matter of curative or symptomrelieving treatments, of responding to acute and urgent needs of patients. Nowadays, with the increase of people with (risk factors for) a chronic condition, there is more emphasis on prevention and care instead of cure to meet the specific needs of this patient population (Wagner et al., 2005). Care for people with a chronic condition goes beyond the medical treatment and has to focus on educational, behavioural and psychosocial needs as well (Wagner, Austing, \& Von Korff, 1996). An important characteristic of care for chronically ill people is that it mainly takes place outside health care settings during daily life of patients and treatment outcomes depend heavily on the patients' self-care activities. The patients' key role in the management of their disease is referred to as self-management and consists of behavioural, cognitive, and emotional responses to a constantly changing state of one's condition (Barlow et al., 2002). With an emphasis on self-management, chronic care does a great appeal to patients' responsibilities. This also leads to a change in the patient's role during health care consultations; both health care professionals and patients are seen as experts (Funnell \& Anderson, 2004). Whereas a more paternalistic model of care may be effective in acute medical care, a more patient-centered or empowering approach is needed for the care for chronically ill people. Patients need to feel a sense of control and competence regarding health, health care and interactions with the health care 
system (Menon, 2002) and the treatment needs to suit a patient's personal situation, needs and wishes to obtain long-term results. A patient-centered approach with active patient participation and informed shared decision making is believed to improve self-management outcomes (Funnell \& Anderson, 2002; Rollnick et al., 1999; Stewart, Brown, Donner et al., 2000; Van Dam et al., 2003; Williams et al., 2005).

The importance of self-management and patient-centered care for people with a chronic condition is widely recognized (Barlow et al., 2002; Funnell, 2006; Newman et al., 2004; Tomky, Cypress, Dang et al., 2008) and is reflected in large numbers of intervention studies among people with different chronic diseases. There is an ongoing search for optimal health care strategies that suit the specific demands of care for chronically ill people, which can limit the consequences of the condition for patients in terms of health outcomes and quality of life and for the society in terms of costs. The research described in this dissertation is only one out of many initiatives. Previously it has been investigated if the care for stable type 2 diabetes patients by the internist or general practitioner could be substituted by care from a nurse specialist. Evaluations revealed that this substitution model was appropriate for these patients (Houweling, 2005; Vrijhoef et al., 2001) and nowadays, in the Netherlands, practice nurses are responsible for the daily care of patients with a chronic disease such as type 2 diabetes, asthma/COPD, or hypertension (Lamkaddem, De Bakker, Nijland et al., 2004), under the supervision of a general practitioner. Also, educational interventions in groups, based on the empowerment approach have been developed and shown to be effective for health outcomes of diabetic patients (Davies, Heller, Skinner et al., 2008; Keers et al., 2006). Besides diabetes studies, there are numerous self-management intervention studies among patients with asthma showing effects on knowledge, compliance with medication and symptom management (Barlow et al., 2002). What is more, interventions are not only addressed to chronically ill patients, but also to people being at risk for a chronic disease, for example for the prevention of cardiovascular diseases (CVD). For instance, an intervention consisting of a risk assessment and risk communication followed by a Motivational Interviewing (MI)-based consultation was recently evaluated (Koelewijn-Van Loon, Van Steenkiste, Ronda et al., 2008). Although no benefits of the intervention above usual care were found in terms of lifestyle outcomes and cardiovascular risk, patients were more satisfied with the communication and had a better risk perception at short term follow-up (Koelewijn-Van Loon, Van der Weijden, Ronda et al., 2010). Also, computer 
tailoring interventions and telephone $\mathrm{MI}$ interventions have been evaluated as strategies to prevent CVD (Van Keulen, Mesters, Brug et al., 2008).

Despite the various initiatives to improve self-management and to optimize health care strategies, no obvious best practice has emerged. The research described in this dissertation is about supporting self-management behaviour of patients with type 2 diabetes (DM2). A dual strategy, consisting of a web-based education programme and a Motivational Interviewing (MI)-based counselling training for practice nurses was developed and evaluated. Additionally, a systematic review of recently published reports on self-management interventions was carried out.

\section{The intervention}

As a result of our project, a Dutch diabetes education programme (DIEP) is developed and permanently available via the World Wide Web (www.diep.info) for anyone who is interested. Moreover, the content of this programme is continuously renewed, extended and adapted based on evaluations among users of the programme. As described in Chapter 3, the programme can improve diabetes knowledge and it is highly valued, not only by DM2 patients, but also by people in the field of diabetes care in the Netherlands; the programme was awarded with two prizes, the quality award for diabetes care 2005 and the Diabetes Education Study Group education award 2009. Furthermore, in 2006 the Netherlands Institute for Health Promotion and Disease Prevention (NIGZ) assessed the quality of diabetes education materials in the Netherlands including DIEP, which was described as a highly promising tool (Looise et al., 2006). This reflects a high acceptability of the programme among the primary (patients) and secondary (health care professionals) target populations which can be attributed to the specific features of the programme. Although web-based programmes are only available for people with access to the Internet, the Internet penetration rate was $90 \%$ in 2009 in the Netherlands and still increases (CBS, 2009). Moreover, the number of people using Internet for information about health is growing (Kaiser Family Foundation, 2005) and the advantages of online education are numerous. The content of the programme can relatively easily be changed and updated, there are interactive possibilities and the programme can be extended to other chronic conditions. E-health technology becomes increasingly important in the management of chronic conditions. The number of people with one or more chronic diseases increases and the amount of time a health care professional has per patient will probably decrease. Besides, more emphasis is placed on 
active patient participation and shared decision making, putting additional demands on the health care system. Therefore, supportive tools for adequate self-management, supporting health care professionals and patientprofessionals interaction, are needed. E-health technology fits these needs and previous research has shown potential profits such as assisting patients in achieving individualized goals and facilitating the collaboration between patients and health care professionals (Verhoeven, Tanja-Dijkstra, Nijland et al., 2010). However, E-health technology and web-based education programmes like ours are no panacea; there are also some important disadvantages which should be taken into account. Providers of web-based programmes have no control of how these programmes are used by the target population, of the frequency of use, of the exposure time, and there is no control of how people process the information found which can lead to misconceptions with needless fear or wrongful relief as consequence (De Nooijer, Oenema, Kloek et al., 2005). Therefore, E-health technology cannot replace direct contact between patients and health care professionals; interpersonal contact remains indispensable for more tailored information and advice and for an appropriate explanation of information to individual patients.

Effective implementation and adequate use of technologies like our webbased programme require initiative and action from patients and health care professionals. To make the nurses in our study responsive to the selfmanagement supportive features of our education programme and to improve counselling skills, the second component of our intervention comprised a Motivational Interviewing (MI)-based counselling training in which strategies like agenda setting and working towards concrete goals were addressed. The most empirical support for MI has been found in the field of substance use (Ball, Martino, Nich et al., 2007; Burke et al., 2003; Dunn et al., 2001; Hettema et al., 2005) but nowadays, MI is increasingly being advocated in other health areas such as diet, exercise, and diabetes (Hettema et al., 2005; Miller \& Rose, 2009; Resnicow et al., 2002). We created an adaptive form of MI based on Motivational Interviewing and Health Behavior Change counselling (Miller \& Rollnick, 2002; Rollnick et al., 1999) to fit the counselling strategy to the quarterly consultations in DM care. Even though the education programme was positively evaluated and an MI counselling strategy seems promising for changing health behaviours, the effect evaluation of our dual strategy showed limited results in terms of patients' health (behaviour) outcomes. 


\section{MI-based counselling in diabetes care}

MI is a client-centered, directive method for enhancing intrinsic motivation to change (Miller \& Rollnick, 2002) and with our education and counselling strategy we aimed to increase patient participation and to teach specific counselling skills for nurses to improve patients' self-management and related health (behaviour) outcomes. However, we observed a limited level of use of the counselling strategy and according to the nurses in our study the strategy was not applicable during every consultation and not for every patient. MI approaches are merely focused at and useful for resolving ambivalence and eliciting change talk. However, self-management behaviour is only one topic to be addressed during usual care visits besides a whole range of other topics such as evaluation of the present medical condition, medication prescriptions or more psychosocial issues (Doherty \& Roberts, 2002). We believe that a limited implementation of MI does not mean that nurses failed to apply a clientcentered approach during their consultations. Patient-centeredness may result in a shared decision not to change any behaviour or even not to discuss behaviour change if other topics are more relevant at that moment. In this situation, an MI approach does not seem indicated. MI-based counselling may be specifically relevant for diabetes consultations in which self-management is the main topic to be discussed. This idea was supported by the fact that some nurses in our study argued that the strategy was easier to use in smoking cessation consultations.

In contrast to our intervention, in previous research most interventions consist of specific MI-sessions apart from usual care consultations and these sessions are often solely targeted at behaviour change (Ismail et al., 2008; Smith-West et al., 2007) which may have enhanced the applicability of the strategy. Previous research of MI in diabetes care is very limited and although some studies show beneficial effects on glucose levels, physical activity, and weight (Martins \& McNeil, 2009), the empirical support for the effectiveness in this area is meagre which indicates that MI in chronic disease care may require a different approach than in addictive behaviours (Resnicow et al., 2002). Thus, although MI has gained popularity among health care professionals in the Netherlands, the applicability and added value in regular diabetes consultations needs further exploration. 


\section{Implementing a new education and counselling strategy in quarterly consultations}

Implementation issues are the most probable explanation for the limited effects of our counselling and education strategy. No structural implementation of our education programme took place and also the use of MI-based skills was poor. A complicating (but also a realistic) characteristic of our intervention is that the counselling strategy had to be implemented in regular day-to-day practice. In our review (Chapter 6) we found that other studies targeted at improving patients' self-management merely evaluate interventions that add separate sessions, additional to usual care; the same holds for MI studies in diabetes care (Martins \& McNeil, 2009). Implementing MI interventions in 'real world' settings with health care professionals who have their own routines (Carroll et al., 2002) and who are influenced by treatment protocols and/or by reimbursement systems (Emmons \& Rollnick, 2001) is difficult. A feasibility study described by Doherty and colleagues revealed barriers for diabetes staff members to apply new MI-based counselling competences (Doherty, Hall, James et al., 2000). As in our study, they found that time pressure and the dominance of biomedical aspects of consultations hampered the implementation of competences. When implemented in usual care (and not on top of that), professionals have to replace their routine behaviour with a new approach. In an implementation study outside diabetes care but with usual care counsellors, it was found that a 2-day MI workshop can increase the frequency of some practice behaviours consistent with MI, but could not alter prior counselling habits (Miller \& Mount, 2001). Where health care professionals in our focus group declared that it is difficult to change patient behaviour (Heinrich et al., 2009), we have experienced that changing nurses' behaviour is as difficult as changing patients' behaviour. Drawing general conclusions about the effectiveness of MI-based interventions in diabetes care based on our effect evaluation would be invalid since there is a considerable risk of a type III error; evaluating a program that has not been adequately implemented (Basch et al., 1985). Therefore, although we failed to present convincing evidence for the additional benefits of MI-based counselling above usual care, we cannot conclude that MI has no added value in diabetes care.

\section{Factors related to patients' health outcomes}

Outcomes for patients with chronic illnesses are directly influenced by medical treatment and self-management, which in turn are affected by health care factors as well as environment and context factors such as patient 
characteristics and community and social context (Glasgow, 1995). Interventions to improve outcomes can be targeted at different facets such as provider-oriented components, organizational changes, information systems changes, and patient-oriented interventions.

Our intervention was focused on two facets, i.e. a provider-oriented component, the MI-based counselling training, and a patient-oriented component, the web-based education programme. Counselling and education are crucial in chronic care; however these are by far not the only factors influencing the outcomes. Despite the advantage of addressing two categories compared to other interventions that are often focused at one category, our project can be improved by the development of interventions targeted at information systems changes and organizational changes.

The intervention described in this dissertation can be seen as a starting point for a larger project, in which more attention should be paid to the implementation of the current strategy and covering the other two intervention categories as well in order to improve patients' health outcomes and quality of care. In part, we started extending our project already by the development and implementation of the training diep@work for health care professionals. For further development, the DIEP foundation was established in September 2008. Until now, the intervention was not targeted at information systems changes. But one of the aims of the foundation is to enable ongoing development of the web-based programme based on the needs of patients and health care professionals. Besides the course diep@work, two new initiatives are launched based on previous research about e-health, to increase the interactivity of the programme and thereby stimulate information exchange. Another idea for further development of the programme is to link our education programme to the electronic patient data system to stimulate information exchange between patients and health care professionals.

The second facet that was not addressed in our project concerns organizational changes. These are beyond the scope of the DIEP foundation and need guidance from a national level, but our project revealed valuable information indicating opportunities to improve the organization of diabetes care. For instance, the intention of three-monthly consultations with practice nurses was to offer better education and counselling opportunities besides the merely medical care that general practitioners provide (Vrijhoef et al., 2001). Nowadays, nurses have to take responsibility for the medical treatment of diabetes (under supervision of the general practitioner) and for patient education and counselling, without passing over a patients personal agenda and general wellbeing. Nurses' way of working is influenced by patient data 
recording systems which have a somewhat anamnestic character, and sometimes even two systems have to be used simultaneously by the nurses. Although these data files may be necessary for the communication between health care professionals and for the evaluation and justification of medical care costs, they might interfere with the education and counselling tasks besides medical examinations and assessments. Furthermore, there is a lack of abilities to reflect on the quality of counselling since patients' clinical outcomes and patient satisfaction are often the only parameters of nurses' performances. The quality of counselling therefore mainly depends on the individual efforts and personal interests of practice nurses. These and other aforementioned factors may explain the implementation difficulties. Other intervention studies anticipated on this common problem by offering the intervention as separate counselling sessions, additional to usual care visits (Ismail et al., 2008; Smith-West et al., 2007). This is a somewhat contradictory situation compared to the initial goal of quarterly diabetes consultations by nurses who specialize in chronic care. Therefore, education and counselling tasks besides medical examinations and assessments needs guaranteed priority, or the interpretation of nurses tasks need to be reconsidered. An alternative would be to offer separate counselling sessions besides quarterly consultations. However, this imposes an extra burden on the diabetes care capacity which is already limited because of the increased number of patients.

\section{Methodological considerations}

We developed, implemented and evaluated an evidence based education and counselling strategy in an actual practice setting. A strong element of the development of our education programme was the collaboration between programme planners, Dutch diabetes organisations and potential users. This prolonged and complicated the developmental process but we are convinced of the importance of this teamwork for the quality of the content and for the national support of the programme.

A characteristic of the current project is the use of different methods to evaluate the intervention which strengthened the research (Richards \& Hamers, 2009). For the effect evaluations, an online questionnaire as well as written questionnaires were used among the patient population but also nurses had to collect and record clinical data measured in a predetermined time period. More important, besides effect evaluations, quantitative and qualitative process evaluations were carried out among patients and nurses of the experimental and the control group which are often lacking alongside RCTs (Lewin, Glenton, \& 
Oxman, 2009). The methods used were online evaluation forms, one-on-one and telephone interviews, and audio-taped consultations scored by two trained independent research assistants. Collecting and analyzing these different kinds of data was time consuming and required an accurate task organization including the use of multiple announcements and reminders, but the different methods yielded valuable data for our evaluation.

There are several methodological considerations regarding research designs, measures used, the intervention and participants of the studies presented in this dissertation which should be considered in interpreting the outcomes of our studies. These are described below.

\section{Research designs}

The first issue relates to the follow-up time. As described in Chapter 3, in the effect evaluation of the web-based education programme the post-test measure of knowledge followed after two weeks of access to the programme. A longer access period with a longer follow-up would have been more ideal. Before and during this trial, people needed a login code to have access. This allowed us to perform a randomized controlled trial with a control group that had no admission. This experimental study design is seen as the most reliable and recommended method of evaluating interventions (Rossi, Freeman, \& Lipsey, 1999; Windsor, Baranowski, Clark et al., 1994). However, it was decided to remove the login procedure to enable nation wide access and therefore we unfortunately had to finish our study on a short term. Conversely, for the effect evaluation of our education and counselling intervention in total, as described in Chapter 4, a much longer follow-up time was planned. Post-tests were conducted after 12 and after 24 months. Since most patients visit their nurses only quarterly according to the Dutch diabetes care guidelines (Diabetesvereniging Nederland, 2008; Nederlandse Diabetes Federatie, 2007), this follow-up time was necessary to be able to detect changes in selfmanagement and in clinical and process outcomes.

The second issue concerns the randomization procedure. Despite the practice-based character of our project instead of a more experimental setting, we used a randomized design. Preferably, randomization is done at the individual level but this was impossible since we could not expect nurses to apply the new counselling style to only a random selection of their patients. Furthermore, nurses within a general practice but also nurses within a district frequently contact each other and have shared training sessions. Therefore, cluster randomization at district level was the best possible solution to handle the potential threat of contamination. Because of this design, in the effect 
evaluation we corrected for differences between patients of different practices if necessary.

As described above, our research was not limited to effect evaluations but also contained implementation research and user evaluations. These data refined the interpretation of the effect evaluation outcomes and provided insight into implications for ongoing intervention development and practice (Bouffard, Taxman, \& Silverman, 2003; Saunders, Evans, \& Joshi, 2005). The differences in the level of implementation in our study were too small (low level of use in the majority) but it would be interesting to analyze interactions between the level of implementation and the intervention effects in future studies.

\section{Measures}

Considerations regarding the measures used include the use of self-developed and self-reported questionnaires and the fact that we did not measure changes in medication use.

First, we made use of self-developed questionnaires to measure diabetes knowledge in both effect evaluation studies. To our knowledge there was no validated questionnaire that is applicable to the Dutch situation and due to time constraints and available resources it was not possible to perform a validation study. Therefore, we cannot draw any conclusions about the general level of diabetes knowledge. However, pre- and post-test scores can be utilized to assess improvements in DM knowledge as we did.

Second, to assess self-management behaviours self-report questionnaires were used which may have caused bias (Green \& Lewis, 1986). People's biased recall of past behaviour, their limited ability to estimate amounts of nutrients, and socially desirable answers are serious threats to the validity of the studies. Biomarkers could help to overcome these biases but biomarkers, for example for vegetable intake, are not only influenced by nutrition alone but also by biological factors such as metabolism and absorption (Mayne, 2003). Therefore, biomarkers as outcome parameters in this context have also been criticized (Bogers, Van Assema, Kester et al., 2004). Furthermore, analyses of more objective clinical outcomes resulted in similar conclusions in our evaluations and therefore we believe the consequences of possible self-report bias were limited.

Effect evaluation articles often do not describe changes in medication use. However, this could reveal essential information for drawing conclusions about the effectiveness of an intervention. For example, improvements of clinical outcomes could be wrongly attributed to intervention effects whereas changes 
in medication are in fact the cause of improvement. We did not measure changes in medication use because changing medication was no specific topic of our intervention and changes of national medication guidelines would affect patients of the control group and patients of the experimental group. Furthermore, it is unlikely that medication changes biased our outcomes since we found very limited changes on both, clinical and behavioural outcomes. Though, medication use during trials should be measured in future studies to optimize the interpretation of clinical outcomes especially when medication is one of the intervention topics like some of the interventions described in studies in our review (Kim \& Oh, 2003; Skelly et al., 2005).

\section{Intervention}

The implementation of our intervention was suboptimal. Changing nurses' behaviour is as challenging as changing patients' behaviour (Rollnick, 2001) and the number of training activities may have been too limited. However, offering a more intensive training probably would have resulted in a very low nurses' response rate. If MI appears to be effective in future studies in diabetes care, MI counselling strategies could be further incorporated in existing health counselling modules in regular courses for nursing students which would limit the additional MI training intensity.

Also the time between patient visits may have been too long and the time per visit to spend on self-management changes may have been too limited. In accordance with the Dutch diabetes care guidelines, the patients in our study had quarterly consultations in which all aspects of diabetes treatment had to be addressed. As mentioned before, previous MI studies in diabetes care offer specific MI sessions which automatically results in more exposure time and in addition, the time between sessions is often shorter (Channon, Smith, \& Gregory, 2003; Ismail et al., 2008; Knight, Bundy, Morris et al., 2003) or participants receive follow-up calls between sessions (Clark \& Hampson, 2001). Although even a small dose of MI can be effective (Rubak et al., 2005), higher treatment doses tend to increase the effectiveness (Burke et al., 2003; Rubak et al., 2005).

A third methodological consideration is that the intervention is multifaceted in several ways. Not only two different components were combined in one intervention strategy, the components themselves also contained several evidence-based elements. Moreover, the intervention was not targeted at one specific outcome measure but at improving self-management in general with a variety of different outcomes for example fat-intake, smoking behaviour, health locus of control, self-efficacy, HbAlc or blood pressure. This multi-facetted 
character of our intervention complicated the evaluation and it is difficult to attribute (the lack of) effects to particular intervention elements. On the other hand, this type of interventions mirror the complexity of self-management patients are faced with.

\section{Participants}

Considerations about participants address the diversity in needs, the possibility of self-selection bias, the number of participants and the generalizability of our results.

In our studies, the inclusion of participants was not restricted to patients with suboptimal disease parameters. In both effect evaluations described in this dissertation, baseline knowledge scores were relatively high, leaving little room for improvement. Also, as shown in Chapter 4, multiple baseline values of behavioural (fruit and vegetable consumption), clinical (HbA1c and blood pressure) and process outcomes (autonomy support) were already (near) optimal. Less optimal were body mass index and lipid profiles and also more than one third of the population did not meet the physical activity norm score. Although some outcomes could be better, improving self-management may not be a priority for patients who already have optimal values on multiple (but not all) outcomes. A somewhat similar remark was raised by participating health care professionals who argued that the counselling strategy is not applicable in all consultations since the focus is sometimes more on checking the current status without a thorough exploration of a patient's motivation, especially when most medical parameters are satisfactory.

Our intervention was developed to improve self-management, but patients vary in their personal need to improve self-management even when progress could be made. Patients' needs depend partly on their current medical status but also on their personal preferences and situation which can be illustrated by a few examples. First, recently published data among DM2 patients revealed that less than half of all patients are willing to take their medication until all treatment goals are met and that one third of the participants in that particular study (total $\mathrm{n}=994$ ) did not want to take their own responsibility as much as possible (Gorter, Tuytel, de Leeuw et al., 2010; Rutten, 2010). Second, health care professionals who have been using our education programme asked us to adapt the programme to an immigrant population (mainly Turkish and Moroccan people). In a separate study subsidized by the Dutch Diabetes Foundation as part of the project 'Stem van de patient', we explored the need of a programme like ours among this population $(n=133)$ but found out that this programme does not sufficiently fit their needs and possibilities. It was 
therefore decided not to adapt the programme to this specific population at this moment in time. These findings together with the results of previous studies stress the importance of tailored counselling and education. The needs of a diverse patient population cannot be fulfilled by one single education and counselling strategy.

The second issue concerns the possibility of self-selection bias which refers to systematic differences in characteristics between selected and non-selected participants in a study. All participants in our study, patients and health care professionals, were self-selected and data about non-participants are not available. The health care professionals in our study could be more interested in new counselling techniques than non-participants. However, randomization took place after the inclusion of nurses and therefore the selection bias is assumed to be equal to both groups. Also, including less motivated nurses would probably not have led to different conclusions since we found limited effects and a suboptimal implementation. Selection bias could also have occurred during patient recruitment. Nurses were asked to select patients based on our in- and exclusion criteria. The number of patients that were invited to participate varied among general practices and not all variance could be attributed to differences in the size of the practice population. It is possible that nurses selected patients on additional criteria besides our criteria but it is not known if they selected for example patients with a specific interest in selfmanagement or particularly those patients with difficulties in self-management. Finally, patients are self-selected and this may have threatened the external validity of our study. In the evaluation study of the education programme it seems likely that patients were selected who are interested in information about diabetes. This may explain the relative high baseline knowledge scores, but we still were able to demonstrate a significant intervention effect. In the evaluation study of our counselling strategy it seems likely that patients participated who have no obvious resistance towards self-management. However, we did not ask any specific action from the patients themselves except to fill out a questionnaire three times in two years and therefore we believe the bias in our study is limited compared to the bias in for example studies with a selfmanagement intervention consisting of educational group sessions. The fact that we did not specifically ask patients to participate in a self-management intervention but only asked them to cooperate with our evaluation by filling out questionnaires may be an additional explanation for the lack of effects compared to other intervention studies. It is likely that our study sample included patients without a personal need to improve self-management which 
reflects the general patient population more adequate but decreases the likelihood to show beneficial effects from the intervention.

A loss of power was a serious threat to the quality of the evaluation study of the counselling strategy. Because of our design, drop-out from one single nurse automatically resulted in a drop-out of a whole group of patients, except in some practices where more than one trained nurse worked. Because of this characteristic together with the relative long follow-up period of two years we decided beforehand to anticipate a serious loss of power and therefore recruited more nurses and patients than necessary according to the power calculation. This complicated the logistic management of our trial but, besides decreasing the risk of insufficient power, this decision had another advantage; the external validity of our study was increased by including more different general practices in more different regions of the Netherlands. Practices from rural areas as well as from urban areas were included, covering a diverse population. We were not able to take regional differences into account in our analyses since we randomized on district level, but we did correct for differences between practices when necessary.

\section{RECOMMENDATIONS FOR FUTURE RESEARCH}

The research described in this dissertation not only provided information on development, effects and implementation of our intervention but also generated several ideas for future research on self-management interventions, for MIbased interventions, and for future developments of our project.

\section{Evaluating self-management interventions}

As described in the introduction of this dissertation, diabetes self-management is complex and previously many interventions to improve self-management have been developed and evaluated. Our review (Chapter 6) showed beneficial effects of self-management interventions but intervention studies are often primarily focused on behavioural and clinical outcome measures. Process measures are often omitted or not described whereas the processes underlying behaviour change are still unclear for example for improving the amount of physical activity (Heinrich, Schaper, \& De Vries, 2010; Norris et al., 2001). Also, the application of MI interventions in chronic disease care is relatively new and although there is evidence that eliciting so-called "change talk" and applying the therapeutic skill of empathic understanding promotes positive 
changes, the theoretical components and the actual processes underlying changes are still unclear (Markland, Ryan, Tobin et al., 2005; Miller \& Rose, 2009). The process measures we measured were perceived autonomy support, self-efficacy, health locus of control and diabetes knowledge. The changes made by our intervention however were too small for an exploration of behaviour change processes.

Our recommendation for future research is therefore to report on process outcomes and interactions between different outcomes to obtain a better understanding of the underlying processes of change.

\section{MI-based interventions}

At present, most evidence for the effectiveness of MI interventions is found for people with addictive behaviour, for example for people with alcohol problems (Britt et al., 2004). However, there is less convincing evidence for the use of MI in other health areas such as diet, exercise and diabetes since this is a relatively young research area (Dunn et al., 2001; Martins \& McNeil, 2009; VanWormer \& Boucher, 2004). Lifestyle counselling and self-management counselling differ in several ways from counselling people with an addiction (Resnicow et al., 2002). The meaning and the impact of behaviours may be quite different, for example between vegetable consumption and heroin use; also the required changes differ largely. In case of addictive behaviour, elimination of behaviour is required whereas in case of chronic disease behaviours the change process may involve modification or addition. More research is necessary regarding the effectiveness of MI based interventions in the context of self-management counselling for people with a chronic condition.

Furthermore, more research is necessary addressing interventions in realworld settings with health care professionals from the field instead of intensively trained MI interventionists or psychologists. Most studies involving usual care health professionals are primarily focused on training issues and do not examine the effect of the training on client outcomes (Madson et al., 2009). Also, as described earlier in this chapter, many self-management or MI interventions take place outside usual care visits, for example home visits by a nurse (Skelly et al., 2005), a 2.5 day retreat followed by weekly group meetings (Toobert et al., 2003) or individual MI sessions by a licensed clinical psychologist (Smith-West et al., 2007). In this type of studies, the question is what will happen with the intervention when the trial is finished. Is it feasible to offer additional counselling sessions to all diabetic patients besides the 
health care visits patients already receive? With the increase in the number of patients suffering from diabetes, the answer to this question will probably be negative. Interventions which can improve the existing clinical practice are necessary and therefore, more evaluation studies altering usual care counselling behaviour are indispensable. Knowledge should be gained about adequate training and skill levels of health care professionals. A systematic review addressing training in MI has been published before (Madson et al., 2009). However, the authors failed to draw conclusions about the effectiveness of different types of training methods and about the optimal amount of training time. This is partly because training activities are often minimally described. But more important, training methods did not vary enough to draw conclusions about the effectiveness of different methods. The most often used methods for training were through didactic instruction and experiential exercises. In contrast to our training, the majority of courses contained a workshop format without further supervision or feedback. Only one study described ongoing coaching. From our implementation study, as well as from other research (Miller \& Rose, 2009; Miller, Yahne, Moyers et al., 2004; Walters, Matson, Baer et al., 2005) it can be concluded that individual feedback and follow-up coaching are essential. More research specifically about MI training methods is needed to draw conclusions about optimal MI training methods.

In summary, first it should be investigated if MI-based counselling has an added value above current counselling strategies in diabetes care. If an added value has been proven, subsequent evaluation studies should target MI training methods for health care professionals of the field. Finally, the implementation of MI in usual care should be examined to answer questions like 'Can MI be implemented in regular consultations or are separate MI counselling sessions needed?'.

\section{Future developments of our project}

In order to offer implementation support for health care professionals who want to use the web-based education programme, a course called 'diep@work' was developed. The effect of this course on the implementation of the programme should be explored. In addition, if this course indeed facilitates a more structural use of the programme it becomes relevant to evaluate the effectiveness of the programme; not only in its ability to improve knowledge, but also in the ability to support the communication between health care professionals and patients, to stimulate active patient participation, and to improve adequate self-management. 


\section{PRACTICAL IMPLICATIONS}

Based on the research described in this dissertation, no strong conclusions can be drawn about the (in)effectiveness of MI-based counselling in diabetes. However, we did show beneficial effects of the web-based education programme on knowledge. The implications for practice are twofold; on the one hand, organizational changes are needed to support effective patient education and counselling and on the other hand, the content of our intervention could be improved.

Concerning organizational changes, practice nurses in diabetes care are faced with the difficulty of combining various tasks during one consultation. Medical management is of course a fundamental aspect of diabetes care, but in addition, a more structural emphasis should be placed on other quality aspects of diabetes care, namely on education and counselling to support patients in adequate self-management and active participation. Currently, feedback on clinical outcomes of diabetes care is often realized but not on the quality of education and counselling. This deficiency is also recognized by the Dutch Diabetes Federation (NDF) that started a nationwide initiative in 2009 called 'National Action programme Diabetes (NAD)' (Nederlandse Diabetes Federatie, 2010), anticipating on changes in diabetes care. The leading motive of the NAD is a need for effective prevention and good quality care to decrease the growing burden of diabetes. The primary target of the NAD is a structural implementation of the completed and actualized NDF health care standard as guide for high quality diabetes prevention and diabetes care. Until recently, diabetes self-management, education and lifestyle interventions did not appear in the NDF health care standard, but this will be changed by the NAD and thereby, hopefully a more structural emphasis will be placed on these issues in daily care. Besides these positive developments, the question remains how nurses should combine their different tasks during consultations. Education and counselling require an individually tailored approach. Patients needs vary between different patients but also between different moments in time. A more flexible organization of care in which the length, frequency and content of consultations may vary depending on patients' needs would offer nurses the opportunity to tailor health care. Furthermore, the quality of diabetes care would benefit from supervision on education and counselling skills. At present, no supervision is provided and from our study it appeared difficult for nurses to assess their own functioning. Supervision can improve the quality of care and additionally can provide peer support in acquiring new skills. 
The second part of practical implications addresses the improvement of our intervention. As noted before, the DIEP foundation was established to enable ongoing development and implementation of the education programme. For a nationwide and better implementation, the programme needs to be more widely known and health care professionals need to know how to make optimal use of the programme. This is, and will be realized by the certified diep@work training primarily targeted at nurses. Furthermore, the content of DIEP should become more complete and the programme needs more interactive features that facilitate its use and that can more directly support self-management. The DIEP foundation already started with extending the content of the programme, for example with a specific module about getting started with insulin therapy. Improvements of interactive features of the programme by making use of the advantageous possibilities of e-health technology are also planned. For example, a smart search engine to provide the user with the most appropriate answer for a query could be developed based on previously developed approaches called 'stemming' and 'decompounding' (Hollink, Kamps, Monz et al., 2004). Also a dialogue-feedback system can be developed with standardized dialogues that can be tailored automatically to the goals which patients have formulated. Information about these goals will be stored in a patients' e-diary. The information can be further used to fill in standardized consultation-sheets as preparation for patient-health care professional's face-toface encounters. There are also plans to supplement the web-based programme with a telephone linked care system, which has been proven to be successful to improve self-management behaviour (Friedman, Kazis, Jette et al., 1996), to ensure an ongoing proactive follow-up to stimulate self-management. By adding such features, the programme provides more opportunities for patients and health care professionals to improve patient participation in the health care process and patients' self-management.

As the MI-based counselling strategy concerns; health care professionals should bear in mind that attending a motivational interviewing workshop, even if the course comprises multiple sessions, will not automatically lead to the implementation of an MI-based counselling style. Our training consisted of group sessions and several feedback moments including coaching on the job, but still resulted in a disappointing level of use. In practice it is often impossible to realize prolonged guidance. Health care professionals should be aware of the limited effects of workshops addressing counselling strategies and of the overriding importance of their own efforts in implementation. They need to anticipate the difficulties in daily practice by seeking support from the NAD 
initiative and from colleagues and supervisors to increase the likelihood of successful implementation of new counselling strategies.

In the current project strategies were studied with a patient-centered approach to improve self-management of patients with type 2 diabetes. A newly developed web-based education programme was evaluated and the results show potential benefits of the programme. However, this programme needs to be further developed and special attention should be paid to a more structural implementation in daily practice. Furthermore, a counselling training for practice nurses was developed based on Motivational Interviewing and Health Behaviour Change Counselling. Although considerable training efforts were made, very limited benefits were found, most probably due to inadequate implementation levels. Patient-centered approaches offer opportunities to improve self-management, as shown in our review, but the implementation in daily practice makes high demands on interventions and organization of care. Besides medical aspects, more emphasis should be placed on education and counselling as quality aspect of diabetes care, where feedback from colleagues and supervisors is needed to support health care professionals and to improve the quality of care. 
Samenvatting 
128 
Het aantal mensen met de chronische ziekte diabetes mellitus neemt wereldwijd toe. Deze toename komt voornamelijk door een groei in het aantal mensen met type 2 diabetes (DM2). Er zijn meerdere oorzaken voor het toenemende vóórkomen van DM2; mensen worden steeds ouder, mensen hebben een minder gezonde leefstijl en zorgverleners zijn alerter op bekende risicofactoren voor diabetes waardoor patiënten eerder dan voorheen worden gediagnosticeerd. De gevolgen van diabetes kunnen verstrekkend zijn, waaronder een verhoogd risico op hart- en vaatziekten. Om de consequenties van DM2 voor patiënten en voor de samenleving te beperken, zijn effectieve behandelstrategieën nodig.

De behandeling van DM2 bestaat enerzijds uit medicatie en anderzijds uit zelfmanagement; het individuele vermogen om goed om te gaan met symptomen, behandeling, lichamelijke en sociale consequenties en leefstijlaanpassingen inherent aan leven met een chronische aandoening. Zelfmanagement gaat verder dan adequaat medicijngebruik en gaat ook over het vinden van de juiste balans tussen voedselinname, beweging en het effect van medicijnen. Daarnaast moeten patiënten hun conditie goed in de gaten houden en letten op bepaalde symptomen. Wat ook bij zelfmanagement hoort is het omgaan met de psychologische consequenties van de ziekte, bijvoorbeeld accepteren dat je DM2 hebt en stress management. Diabetes zelfmanagement is complex en vraagt veel van patiënten. Patiënten hebben, net als veel mensen zonder DM2, moeite met gedragsverandering en gedragsbehoud waardoor de gezondheidsstatus vaak suboptimaal is. Goede educatie en begeleiding van het medisch team zijn onontbeerlijk.

Dit proefschrift gaat over de ontwikkeling, inhoud en evaluatie van een educatie- en counselingstrategie, gericht op patiënten en op zorgverleners, om zelfmanagement van patiënten te ondersteunen en daarmee de impact van de ziekte te beperken. De strategie bestaat uit een diabetes educatieprogramma dat beschikbaar is op internet en een training motiverende gespreksvoering voor praktijkverpleegkundigen (praktijkondersteuners en verpleegkundigen).

De ontwikkeling van het educatieprogramma aan de hand van de stappen van het Intervention Mapping protocol en de inhoud van het programma worden beschreven in hoofdstuk 2. Gebaseerd op een behoeftepeiling, bestaande uit een literatuurstudie naar effectieve strategieën ter bevordering van zelfmanagement en focusgroep interviews onder diabetespatiënten en zorgverleners, zijn doelen voor het programma geformuleerd. Het programma beoogt het ziekte-inzicht te vergroten, de communicatie tussen zorgverleners en patiënten te ondersteunen, actieve patiëntbetrokkenheid te stimuleren en hulpmiddelen ter bevordering van zelfmanagement aan te bieden. Gedurende 
de ontwikkeling van het programma was er een structurele samenwerking met vertegenwoordigers van alle relevante Nederlandse diabetesorganisaties en met DM2 patiënten zelf. De planmatige ontwikkeling heeft uiteindelijk geleid tot www.diep.info; een uniek, evidence-based educatieprogramma bestaande uit zeven hoofdstukken met basis- en nadere informatie, een zoekfunctie, zelfmanagement informatieformulieren en een werkboek om doelen te stellen en consulten voor te bereiden. De informatie bestaat grotendeels uit gesproken tekst ondersteund met samenvattende beeldtekst, plaatjes, video's en patiëntenervaringen.

Het educatieprogramma is zowel op effect als meer procesmatig geëvalueerd. De effect- en gebruikersevaluatie worden beschreven in hoofdstuk 3. De effectevaluatie $(n=99)$ toonde aan dat het programma het DM2 ziekteinzicht van patiënten kan vergroten. De gebruikersevaluatie, bestaande uit een online vragenlijst $(\mathrm{n}=564)$ en interviews met patiënten en zorgverleners $(\mathrm{n}=11)$, liet zien dat gebruikers het programma zeer positief beoordelen maar dat het programma hoofdzakelijk wordt gebruikt als informatiebron en niet als hulpmiddel om communicatie, actieve patiëntbetrokkenheid en adequaat zelfmanagement te ondersteunen. Gebaseerd op deze bevindingen is een training voor zorgverleners (diep@work) ontwikkeld om optimaal gebruik van het programma in de dagelijkse praktijk te ondersteunen en daarmee de meerwaarde van het programma te vergroten. Met de implementatie hiervan is reeds gestart.

De tweede en aanvullende component van onze strategie, de training motiverende gespreksvoering voor praktijkverpleegkundigen, was gebaseerd op de principes van Motivational Interviewing (MI) en Health Behaviour Change Counseling. De inhoud en effectevaluatie hiervan wordt in hoofdstuk 4 beschreven. Deze component bestond uit een tweedaagse groepstraining voor verpleegkundigen, gevolgd door individuele feedback en terugkombijeenkomsten. De totale trainingstijd bedroeg ongeveer 21,5 uren en 5 optionele uren voor terugkombijeenkomsten. Gedurende de groepstraining stonden MI principes en technieken en een gespreksprotocol centraal. Daarnaast werd aandacht besteed aan het educatieprogramma en de link tussen kenmerken van het programma en de gesprekstechnieken, zoals het gebruik van het haalbare doelenformulier. De eerste individuele feedback die verpleegkundigen kregen bestond uit een brief met feedback op opgenomen consulten ongeveer 3 maanden na de groepstraining. Naar aanleiding van deze consulten is besloten om meer individuele ondersteuning bij de implementatie te bieden. Ongeveer 6, 9 en 11 maanden na de groepstraining kregen verpleegkundigen directe feedback op hun consultvoering door middel van visitaties gedurende 1 dagdeel 
per keer. De effectevaluatie $(n=584)$ liet geen duidelijke effecten van de training zien op klinische, gedragsmatige en procesmatige uitkomstmaten, behalve een positief effect op kennis en op een van de 'health locus of control' dimensies en een negatief effect op vetinname en HDL-cholesterol. Op basis van deze studie kon niet worden geconcludeerd dat de training een meerwaarde had boven zorg door verpleegkundigen die niet waren getraind.

De meest waarschijnlijke verklaring voor het gebrek aan effectiviteit van de training motiverende gespreksvoering werd gevonden in de implementatiestudie die in hoofdstuk 5 wordt beschreven. Deze studie had als doel de mate van implementatie van de counselingmethode te evalueren evenals het exploreren van factoren die de mate van implementatie hebben beïnvloed. Hoewel zorgverleners aangaven de geleerde vaardigheden vaak toe te passen werd op basis van opgenomen, gescoorde consulten een zeer beperkte implementatie waargenomen. De belangrijkste factoren die volgens verpleegkundigen een rol bij de implementatie hebben gespeeld waren: trainingsintensiteit, er aan herinnerd worden, ervaren werkdruk, beschikbare tijd per patiënt, type patiënt en steun van leidinggevenden en collegae. Uit ons onderzoek bleek net als uit eerdere onderzoeken dat MI-trainingen in de vorm van een workshop onvoldoende zijn voor het aanleren van de methode en dat de rol van individuele feedback en terugkombijeenkomsten essentieel zijn voor het leerproces.

Naast de ontwikkeling en evaluatie van een educatie- en counselingstrategie werd een systematisch literatuuronderzoek naar recent gepubliceerde (20002010) zelfmanagement-interventies uitgevoerd, welke is beschreven in hoofdstuk 6. Veertien studies voldeden aan de inclusiecriteria en zelfmanagement-interventies bleken een duidelijke meerwaarde te hebben voor het verbeteren van voedingsgewoonten, diabetes specifieke kwaliteit van leven en kennis. Meer gemengde resultaten werden gevonden voor beweging en klinische uitkomsten en slechts weinig interventies waren in staat gewicht te verminderen. Interventietype (1-op-1 versus groepsinterventies) en interventiemethode (gericht op leren, op plannen of oefenen / ervaren) bleken geen duidelijk verschil te maken, hoewel groepsinterventies met een oefencomponent de meeste potentie leken te hebben om metabole controle te verbeteren. Conclusies over de onderliggende veranderingsprocessen konden niet worden getrokken omdat procesmaten vaak niet gemeten of gerapporteerd werden en de wel gerapporteerde maten varieerden sterk. Kenmerkend voor de interventies was dat didactische leermethoden nauwelijks werden toegepast, terwijl dat in studies voor 2000 nog wel het geval was. Wat verder opviel was 
dat op één interventie na, alle interventies plaatsvonden buiten de dagelijkse diabeteszorg.

In hoofdstuk 7 tot slot wordt het onderzoek samengevat en worden de belangrijkste uitkomsten geïntegreerd en bediscussieerd. Tevens worden de methodologische kenmerken van de studies in beschouwing genomen, gevolgd door een beschrijving van aanbevelingen voor verder onderzoek en voor de praktijk. Toekomstig zelfmanagement-onderzoek dient meer aandacht te besteden aan procesmaten om beter inzicht te krijgen in processen die ten grondslag liggen aan gedragsverandering. Motiverende gespreksvoering als manier van counseling is populair, maar diabeteszorgverleners dienen zich ervan bewust te zijn dat voor het toepassen van deze counselingstijl meer nodig is dan het volgen van een 2- of 3-daagse workshop. Bewijs voor de effectiviteit van motiverende gespreksvoering binnen diabeteszorg is nog beperkt en verdient verdere aandacht. Ook moet gekeken worden naar de effectiviteit van verschillende trainingsmethoden en de toepasbaarheid van de methode in de huidige organisatie van zorg. Naast het medische aspect dient in de praktijk meer nadruk te worden gelegd op educatie en counseling als kwaliteitsaspect van diabeteszorg, waarbij intervisies zorgverleners dienen te ondersteunen en de kwaliteit van zorg kunnen bevorderen. Tevens kan de inhoud van het educatieprogramma zoals beschreven in dit proefschrift verder worden geoptimaliseerd, bijvoorbeeld door het educatieprogramma interactief te maken. 
References 
134 
Abraham, C., \& Michie, S. (2008). A taxonomy of behavior change techniques used in interventions. Health Psychology, 27, 379-387.

Adler, A. I., Stratton, I. M., Neil, H. A., Yudkin, J. S., Matthews, D. R., Cull, C. A., Wright, A. D., Turner, R. C., \& Holman, R. R. (2000). Association of systolic blood pressure with macrovascular and microvascular complications of type 2 diabetes (UKPDS 36): prospective observational study. British Medical Journal, 321, 412-419.

Adolfsson, E. T., Walker Engstrom, M. L., Smide, B., \& Wikblad, K. (2007). Patient education in type 2 diabetes: a randomized controlled 1-year follow-up study. Diabetes Research and Clinical Practice, 76, 341-350.

Anderson, R. M., Funnell, M. M., Nwankwo, R., Gillard, M. L., Oh, M., \& Fitzgerald, J. T. (2005). Evaluating a problem-based empowerment program for African Americans with diabetes: results of a randomized controlled trial. Ethnicity and Disease 15, 671-678.

Andreassen, H. K., Bujnowska Fedak, M. M., Chronaki, C. E., Dumitru, R. C., Pudule, I., Santana, S., Voss, H., \& Wynn, R. (2007). European citizens' use of E-health services: a study of seven countries. BioMed Central Public Health, 7, 53.

Assal, J. P., Jacquemet, S., \& Morel, Y. (1997). The added value of therapy in diabetes: the education of patients for self-management of their disease. Metabolism, 46, 6164.

Atack, L., Luke, R., \& Chien, E. (2008). Evaluation of patient satisfaction with tailored online patient education information. Computers, Informatics, Nursing, 26, 258264.

Baan, C. A., \& Poos, M. J. J. C. (2009). Neemt het aantal mensen met diabetes mellitus toe of af? Retrieved July 1, 2010, from www.nationaalkompas.nl.

Ball, S. A., Martino, S., Nich, C., Frankforter, T. L., Van Horn, D., Crits-Christoph, P., Woody, G. E., Obert, J. L., Farentinos, C., \& Carroll, K. M. (2007). Site matters: multisite randomized trial of motivational enhancement therapy in community drug abuse clinics. Journal of Consulting and Clinical Psychology, 75, 556-567.

Barlow, J., Wright, C., Sheasby, J., Turner, A., \& Hainsworth, J. (2002). Selfmanagement approaches for people with chronic conditions: a review. Patient Education and Counseling, 48, 177-187.

Barrera, M., Toobert, D. J., Angell, K. L., Glasgow, R. E., \& Mackinnon, D. P. (2006). Social support and social-ecological resources as mediators of lifestyle intervention effects for type 2 diabetes. Journal of Health Psychology, 11, 483-495.

Bartholomew, L. K., Parcel, G. S., Kok, G., \& Gottlieb, N. H. (2000). Planning health promotion programs. An intervention mapping approach (2nd ed.). San Francisco: Jossey-Bass.

Basch, C. E., Sliepcevich, E. M., Gold, R. S., Duncan, D. F., \& Kolbe, L. J. (1985). Avoiding type III errors in health education program evaluations: a case study. Health Education Quarterly, 12, 315-331. 
Belton, A. (2003). International Standards for Diabetes Education. Diabetes Voice, 48, 17-19.

Benigeri, M., \& Pluye, P. (2003). Shortcomings of health information on the Internet. Health Promotion International, 18, 381-386.

Boezen, H. M., Postma, D. S., Smit, H. A., \& Poos, M. J. J. C. (2006). Hoe vaak komt COPD voor en hoeveel mensen sterven eraan? Retrieved July 1, 2010, from http://www.rivm.nl/vtv/object_class/kom_copd.html.

Bogers, R. P., Van Assema, P., Kester, A. D., Westerterp, K. R., \& Dagnelie, P. C. (2004). Reproducibility, validity, and responsiveness to change of a short questionnaire for measuring fruit and vegetable intake. American Journal of Epidemiology, 159, 900-909.

Bonnet, C., Gagnayre, R., \& d'Ivernois, J. F. (2001). Difficulties of diabetic patients in learning about their illness. Patient Education and Counseling, 42, 159-164.

Bosworth, H. B., Oddone, E. Z., \& Weinberger, M. (2006). Patient Treatment Adherence. Concepts, interventions, and measurement. New Jersey: Lawrence Erlbaum Associates.

Bouffard, J. A., Taxman, F. S., \& Silverman, R. (2003). Improving process evaluations of correctional programs by using a comprehensive evaluation methodology. Evaluation and Program Planning, 26, 149-161.

Boulé, N. G., Haddad, E., Kenny, G. P., Wells, G. A., \& Sigal, R. J. (2001). Effects of exercise on glycemic control and body mass in type 2 diabetes mellitus: a metaanalysis of controlled clinical trials. Journal of the American Medical Association, 286, 1218-1227.

Britt, E., Hudson, S. M., \& Blampied, N. M. (2004). Motivational interviewing in health settings: a review. Patient Education and Counseling, 53, 147-155.

Broers, S., Smets, E., Bindels, P., Evertsz, F. B., Calff, M., \& de Haes, H. (2005). Training general practitioners in behavior change counseling to improve asthma medication adherence. Patient Education and Counseling, 58, 279-287.

Brown, S. A. (1999). Interventions to promote diabetes self-management: state of the science. The Diabetes Educator, 25, 52-61.

Brug, J., Spikmans, F., Aartsen, C., Breedveld, B., Bes, R., \& Fereira, I. (2007). Training dietitians in basic motivational interviewing skills results in changes in their counseling style and in lower saturated fat intakes in their patients. Journal of Nutrition Education and Behavior, 39, 8-12.

Burke, B. L., Arkowitz, H., \& Menchola, M. (2003). The efficacy of motivational interviewing: a meta-analysis of controlled clinical trials. Journal of Consulting \& Clinical Psychology, 71, 843-861.

Carroll, K. M., Farentinos, C., Ball, S. A., Crits Christoph, P., Libby, B., Morgenstern, J., Obert, J. L., Polcin, D., \& Woody, G. E. (2002). MET meets the real world: design issues and clinical strategies in the Clinical Trials Network. Journal of Substance Abuse Treatment, 23, 73-80. 
CBS. (2009). ICT gebruik van huishoudens naar huishoudkenmerken. Heerlen: Centraal Bureau voor de Statistiek.

Channon, S., Smith, V. J., \& Gregory, J. W. (2003). A pilot study of motivational interviewing in adolescents with diabetes. Archives of Disease in Childhood, 88, 680-683.

Charles, C., Gafni, A., \& Whelan, T. (1997). Shared decision-making in the medical encounter: what does it mean? (or it takes at least two to tango). Social Science \& Medicine, 44, 681-692.

Christian, J. G., Bessesen, D. H., Byers, T. E., Christian, K. K., Goldstein, M. G., \& Bock, B. C. (2008). Clinic-based support to help overweight patients with type 2 diabetes increase physical activity and lose weight. Archives of Internal Medicine, $168,141-146$.

Clark, M., \& Hampson, S. E. (2001). Implementing a psychological intervention to improve lifestyle self-management in patients with type 2 diabetes. Patient Education and Counseling, 42, 247-256.

Clark, M., Hampson, S. E., Avery, L., \& Simpson, R. (2004a). Effects of a tailored lifestyle self-management intervention in patients with type 2 diabetes. British Journal of Health Psychology, 9, 365-379.

Clark, M., Hampson, S. E., Avery, L., \& Simpson, R. (2004b). Effects of a brief tailored intervention on the process and predictors of lifestyle behaviour change in patients with type 2 diabetes. Psychology, Health and Medicine, 9, 440-449.

Clement, S. (1995). Diabetes self-management education. Diabetes Care, 18, 12041214.

Cline, R. J., \& Haynes, K. M. (2001). Consumer health information seeking on the Internet: the state of the art. Health Education Research, 16, 671-692.

Cohen, J. (1988). Statistical power analysis for the behavioral sciences. Hillsdale, New York: Lawrence Earlbaum Associates.

Cooper, H., Booth, K., Fear, S., \& Gill, G. (2001). Chronic disease patient education: lessons from meta-analyses. Patient Education and Counseling, 44, 107-117.

Cooper, H. C., Booth, K., \& Gill, G. (2003a). Using combined research methods for exploring diabetes patient education. Patient Education and Counseling, 51, 45-52.

Cooper, H. C., Booth, K., \& Gill, G. (2003b). Patients' perspectives on diabetes health care education. Health Education Research, 18, 191-206.

Craig, C. L., Marshall, A. L., Sjostrom, M., Bauman, A. E., Booth, M. L., Ainsworth, B. E., Pratt, M., Ekelund, U., Yngve, A., Sallis, J. F., \& Oja, P. (2003). International physical activity questionnaire: 12-country reliability and validity. Medicine and Science in Sports and Exercise, 35, 1381-1395.

Davidson, K. W., Goldstein, M., Kaplan, R. M., Kaufmann, P. G., Knatterud, G. L., Orleans, C. T., Spring, B., Trudeau, K. J., \& Whitlock, E. P. (2003). Evidencebased behavioral medicine: what is it and how do we achieve it? Annals of Behavioral Medicine, 26, 161-171. 
Davies, M. J., Heller, S., Skinner, T. C., Campbell, M. J., Carey, M. E., Cradock, S., Dallosso, H. M., Daly, H., Doherty, Y., Eaton, S., Fox, C., Oliver, L., Rantell, K., Rayman, G., \& Khunti, K. (2008). Effectiveness of the diabetes education and self management for ongoing and newly diagnosed (DESMOND) programme for people with newly diagnosed type 2 diabetes: cluster randomised controlled trial. British Medical Journal, 336, 491-495.

DCCT Research Group. (1993). The effect of intensive treatment of diabetes on the development and progression of long-term complications in insulin-dependent diabetes mellitus. New England Journal of Medicine, 329, 977-986.

De Blok, B. M., De Greef, M. H., Ten Hacken, N. H., Sprenger, S. R., Postema, K., \& Wempe, J. B. (2006). The effects of a lifestyle physical activity counseling program with feedback of a pedometer during pulmonary rehabilitation in patients with COPD: a pilot study. Patient Education and Counseling, 61, 48-55.

De Hollander, A. E. M., Hoeymans, N., Melse, J. M., Van Oers, J. A. M., \& Polder, J. J. (2006). Zorg voor gezondheid. Volksgezondheid Toekomst Verkenning 2006. RIVM-rapport nr. 270061003. Houten: Bohn Stafleu Van Loghum.

De Nooijer, J., Oenema, A., Kloek, G., Brug, J., De Vries, H., \& De Vries, N. K. (2005). Bevordering van gezond gedrag via internet: nu en in de toekomst. Maastricht: Maastricht University.

Deakin, T., McShane, C. E., Cade, J. E., \& Williams, R. D. R. R. (2005). Group based training for self-management strategies in people with type 2 diabetes mellitus. The Cochrane Database of Systematic Reviews. Art. No.: CD003417. DOI: 10.1002/14651858.CD003417.pub2.

Deci, E. L., \& Ryan, R. M. (1985). Intrinsic Motivation and Self-Determination in Human Behavior. New York: Plenum.

Diabetesvereniging Nederland. (2008). Diabetes Zorgwijzer. Een overzicht van de zorg voor volwassenen met diabetes type 2. Retrieved June 10, 2010 from www.dvn.nl.

Doherty, Y., Hall, D., James, P. T., Roberts, S. H., \& Simpson, J. (2000). Change counselling in diabetes: the development of a training programme for the diabetes team. Patient Education and Counseling, 40, 263-278.

Doherty, Y., \& Roberts, S. (2002). Motivational interviewing in diabetes practice. Diabetic Medicine, 19 Suppl 3, 1-6.

Drass, J., Kell, S., Osborn, M., Bausell, B., Corcoran, J., Jr., Moskowitz, A., \& Fleming, B. (1998). Diabetes care for Medicare beneficiaries. Attitudes and behaviors of primary care physicians. Diabetes Care, 21, 1282-1287.

Duke, S. A., Colagiuri, S., \& Colagiuri, R. (2009). Individual patient education for people with type 2 diabetes mellitus. The Cochrane Database of Systematic Reviews. Art. No.: CD005268. DOI: 10.1002/14651858.CD005268.pub2.

Dunn, C., Deroo, L., \& Rivara, F. P. (2001). The use of brief interventions adapted from motivational interviewing across behavioral domains: a systematic review. Addiction, 96, 1725-1742. 
Elwyn, G. (2001). Shared decision making. Katholieke Universiteit Nijmegen, Nijmegen.

Emmons, K. M., \& Rollnick, S. (2001). Motivational interviewing in health care settings. Opportunities and limitations. American Journal of Preventive Medicine, 20, 68-74.

Eysenbach, G., Powell, J., Kuss, O., \& Sa, E. R. (2002). Empirical studies assessing the quality of health information for consumers on the world wide web: a systematic review. Journal of the American Medical Association, 287, 2691-2700.

Ferrell, B. R., Grant, M. M., Funk, B., Otis Green, S., \& Garcia, N. (1997). Quality of life in breast cancer survivors as identified by focus groups. Psycho-oncology, 6, 13-23.

Feste, C., \& Anderson, R. M. (1995). Empowerment: from philosophy to practice. Patient Education and Counseling, 26, 139-144.

Festinger, L. (1954). A theory of social comparison processes. Human Relations, 7, 117-140.

Fitzgerald, J. T., Funnell, M. M., Hess, G. E., Barr, P. A., Anderson, R. M., Hiss, R. G., \& Davis, W. K. (1998). The reliability and validity of a brief diabetes knowledge test. Diabetes Care, 21, 706-710.

Fleuren, M., Wiefferink, K., \& Paulussen, T. (2004). Determinants of innovation within health care organizations: literature review and Delphi study. International Journal for Quality in Health Care, 16, 107-123.

Friedman, R. H., Kazis, L. E., Jette, A., Smith, M. B., Stollerman, J., Torgerson, J., \& Carey, K. (1996). A telecommunications system for monitoring and counseling patients with hypertension. Impact on medication adherence and blood pressure control. American Journal of Hypertension, 9, 285-292.

Frosch, D. L., Robert, B. A., \& Kaplan, M. (1999). Shared decision making in clinical medicine: Past Research and Future Directions. American Journal of Preventive Medicine, 17, 285-294.

Funnell, M. M. (2006). The Diabetes Attitudes, Wishes, and Needs (DAWN) Study. Clinical Diabetes, 24, 154-155.

Funnell, M. M., \& Anderson, A. M. (2004). Empowerment and Self-Management of Diabetes. Clinical Diabetes, 22, 123-127.

Funnell, M. M., \& Anderson, R. M. (2002). Working toward the next generation of diabetes self-management education. American Journal of Preventive Medicine, 22, 3-5.

Funnell, M. M., \& Anderson, R. M. (2003). Patient empowerment: a look back, a look ahead. The Diabetes Educator, 29, 454-458.

Gaede, P., Beck, M., Vedel, P., \& Pedersen, O. (2001). Limited impact of lifestyle education in patients with type 2 diabetes mellitus and microalbuminuria: results from a randomized intervention study. Diabetic Medicine, 18, 104-108.

Glasgow, R. E. (1995). A practical model of diabetes management and education. Diabetes Care, 18, 117-126. 
Glasgow, R. E., \& Anderson, R. M. (1999). In diabetes care, moving from compliance to adherence is not enough. Something entirely different is needed. Diabetes Care, 22, 2090-2092.

Glasgow, R. E., \& Toobert, D. J. (2000). Brief, computer-assisted diabetes dietary selfmanagement counseling: effects on behavior, physiologic outcomes, and quality of life. Medical Care, 38, 1062-1073.

Gorter, K. J., Tuytel, G. H., de Leeuw, J. R., van der Bijl, J. J., Bensing, J. M., \& Rutten, G. E. (2010). Preferences and opinions of patients with type 2 diabetes on education and self-care: a cross-sectional survey. Diabetic Medicine, 27, 85-91.

Green, L., \& Lewis, F. (1986). Measurement and evaluation in health education and health promotion. Palo Alto, California: Mayfield Publishing Company.

Guyatt, G. H., Feeny, D. H., \& Patrick, D. L. (1993). Measuring health-related quality of life. Annals of Internal Medicine, 118, 622-629.

Halfens, R. (1985). Locus of Control: Beheersingsorientatie in relatie tot ziekte en gezondheidsgedrag. Limburg University.

Harris, M. I. (2000). Health care and health status and outcomes for patients with type 2 diabetes. Diabetes Care, 23, 754-758.

Heinrich, E., Schaper, N. C., \& De Vries, N. K. (2009). Development of the web-based type 2 diabetes education programme: DIEP. European Diabetes Nursing, 6, 5156.

Heinrich, E., Schaper, N. C., \& De Vries, N. K. (2010). Self-management interventions for type 2 diabetes: a systematic review. European Diabetes Nursing, 7, 71-76.

Heisler, M., Bouknight, R. R., Hayward, R. A., Smith, D. M., \& Kerr, E. A. (2002). The relative importance of physician communication, participatory decision making, and patient understanding in diabetes self-management. Journal of General Internal Medicine, 17, 243-252.

Hettema, J., Steele, J., \& Miller, W. R. (2005). Motivational interviewing. Annual Review of Clinical Psychology, 1, 91-111.

Hollink, V., Kamps, J., Monz, C., \& De Rijke, M. (2004). Monolingual document retrieval for European languages. Information Retrieval, 7, 33-54.

Holman, R. R., Paul, S. K., Bethel, M. A., Matthews, D. R., \& Neil, H. A. (2008). 10year follow-up of intensive glucose control in type 2 diabetes. New England Journal of Medicine, 359, 1577-1589.

Hornsten, A., Lundman, B., Stenlund, H., \& Sandstrom, H. (2005). Metabolic improvement after intervention focusing on personal understanding in type 2 diabetes. Diabetes Research and Clinical Practice, 68, 65-74.

Horsley, D. L., \& Horsley-Loucks, S. (1998). C-BAM brings order to the tornado of change. Journal of Nursing Staff Development, 19, 17-20.

Houweling, S. T. (2005). Taakdelegatie in de eerste- en tweedelijns diabeteszorg. Rijksuniversiteit Groningen, Groningen. 
Hu, F. B., Manson, J. E., Stampfer, M. J., Colditz, G., Liu, S., Solomon, C. G., \& Willett, W. C. (2001). Diet, lifestyle, and the risk of type 2 diabetes mellitus in women. New England Journal of Medicine, 345, 790-797.

Hurn, J., Kneebone, I., \& Cropley, M. (2006). Goal setting as an outcome measure: A systematic review. Clinical Rehabilitation, 20, 756-772.

International Diabetes Federation. (2008). Diabetes Atlas, second edition. Retrieved October, 14, 2008, from www.eatlas.ifd.org.

International Diabetes Federation. (2010). Diabetes Atlas, fourth edition. Retrieved April 13, 2010, from www.diabetesatlas.org.

Internet World Stats. (2008). Countries with highest Internet penetration rates. Retrieved August 5, 2008 from http://www.internetworldstats.com/top25.htm.

IPAQ. International Physical Activity Questionnaires. Retrieved September 25, 2009, from www.ipaq.ki.se/ipaq.htm.

Ismail, K., Thomas, S. M., Maissi, E., Chalder, T., Schmidt, U., Bartlett, J., Patel, A., Dickens, C. M., Creed, F., \& Treasure, J. (2008). Motivational enhancement therapy with and without cognitive behavior therapy to treat type 1 diabetes: a randomized trial. Annals of Internal Medicine, 149, 708-719.

Jarvis, J., Skinner, T. C., Carey, M. E., \& Davies, M. J. (2009). How can structured self-management patient education improve outcomes in people with type 2 diabetes? Diabetes, Obesity and Metabolism, 12, 12-19.

Jerant, A. F., Von Friederichs Fitzwater, M. M., \& Moore, M. (2005). Patients' perceived barriers to active self-management of chronic conditions. Patient Education and Counseling, 57, 300-307.

Jeste, D. V., Dunn, L. B., Folsom, D. P., \& Zisook, D. (2008). Multimedia educational aids for improving consumer knowledge about illness management and treatment decisions: a review of randomized controlled trials. Journal of Psychiatric Research, 42, 1-21.

Kaiser Family Foundation. (2005). E-Health and the elderly: How seniors use the Internet for health - Survey. Retrieved September 12, 2008, from http://www.kff.org/entmedia/entmedia011205pkg.cfm.

Keers, J. C., Bouma, J., Links, T. P., ter Maaten, J. C., Gans, R. O., Wolffenbuttel, B. H., \& Sanderman, R. (2006). One-year follow-up effects of diabetes rehabilitation for patients with prolonged self-management difficulties. Patient Education and Counseling, 60, 16-23.

Kerr, C., Murray, E., Stevenson, F., Gore, C., \& Nazareth, I. (2006). Internet interventions for long-term conditions: patient and caregiver quality criteria. Journal of Medical Internet Research, 8, e13.

Kim, H. S., \& Oh, J. A. (2003). Adherence to diabetes control recommendations: impact of nurse telephone calls. Journal of Advanced Nursing, 44, 256-261.

Kirk, A. F., Mutrie, N., Macintyre, P. D., \& Fisher, M. B. (2004). Promoting and maintaining physical activity in people with type 2 diabetes. American Journal of Preventive Medicine, 27, 289-296. 
Knight, K. M., Bundy, C., Morris, R., Higgs, J. F., Jameson, R. A., Unsworth, P., \& Jayson, D. (2003). The effects of group motivational interviewing and externalizing conversations for adolescents with type-1 diabetes. Psychology, Health \& Medicine, 8, 149-158.

Koelewijn van Loon, M. S., Van der Weijden, T., Ronda, G., Van Steenkiste, B., Winkens, B., Elwyn, G., \& Grol, R. (2010). Improving lifestyle and risk perception through patient involvement in nurse-led cardiovascular risk management: a cluster-randomized controlled trial in primary care. Preventive Medicine, 50, 35-44.

Koelewijn van Loon, M. S., van Steenkiste, B., Ronda, G., Wensing, M., Stoffers, H. E., Elwyn, G., Grol, R., \& van der Weijden, T. (2008). Improving patient adherence to lifestyle advice (IMPALA): a cluster-randomised controlled trial on the implementation of a nurse-led intervention for cardiovascular risk management in primary care (protocol). BioMed Central Health Services Research, 8, 9.

Kok, G., Van den Borne, B., \& Mullen, P. (1997). Effectiveness of health education and health promotion: meta-analyses of effect studies and determinants of effectiveness. Patient Education and Counseling, 30, 19-27.

Krueger, R. A., \& Casey, M. A. (2000). Focus groups: a practical guide for applied research. 3rd Edition. Thousand Oaks, CA.: Sage Publishing.

Kruijver, I. P., Kerkstra, A., Francke, A. L., Bensing, J. M., \& van de Wiel, H. B. (2000). Evaluation of communication training programs in nursing care: a review of the literature. Patient Education and Counseling, 39, 129-145.

Laine, C., \& Davidoff, F. (1996). Patient-centered medicine. A professional evolution. Journal of the American Medical Association, 275, 152-156.

Lamkaddem, M., De Bakker, D., Nijland, A., \& De Haan, J. (2004). De invloed van praktijkondersteuning op de werklast van huisartsen. Utrecht: Nivel.

Larme, A. C., \& Pugh, J. A. (1998). Attitudes of primary care providers toward diabetes: barriers to guideline implementation. Diabetes Care, 21, 1391-1396.

Leffingwell, T. R., Neumann, C. A., Babitzke, A. C., Leedy, M. J., \& Walters, S. T. (2007). Social psychology and motivational interviewing: a review of relevant principles and recommendations for research and practice. Behavioural and Cognitive Psychotherapy, 35, 31-45.

Lewin, S., Glenton, C., \& Oxman, A. D. (2009). Use of qualitative methods alongside randomised controlled trials of complex healthcare interventions: methodological study. British Medical Journal, 339, b3496.

Lipsey, M. W. (1990). Design Sensitivity. Statistical Power for Experimental Research. London New Delhi: Sage Publications.

Looise, B. J., Van der Poel, F. P., \& Bos, V. (2006). Diabeteseducatie in Nederland. State of the art van methoden en materialen. Woerden: NIGZ.

Madson, M. B., Loignon, A. C., \& Lane, C. (2009). Training in motivational interviewing: a systematic review. Journal of Substance Abuse Treatment, 36, 101109. 
Markland, D., Ryan, R. M., Tobin, V. J., \& Rollnick, S. (2005). Motivational interviewing and self-determination theory. Journal of Social and Clinical Psychology, 24, 811-831.

Martins, R. K., \& McNeil, D. W. (2009). Review of Motivational Interviewing in promoting health behaviors. Clinical Psychology Review, 29, 283-293.

Matsuoka, K., Kawata, C., Ikeda, M., Abe, R., \& Atsumi, Y. (1994). Patient education and educational activity in the general public. Diabetes Research and Clinical Practice, 24 Suppl, S279-286.

Mayne, S. T. (2003). Antioxidant nutrients and chronic disease: use of biomarkers of exposure and oxidative stress status in epidemiologic research. Journal of Nutrition, 133, 933S-940S.

Menon, S. T. (2002). Toward a model of psychological health empowerment: implications for health care in multicultural communities. Nurse Education Today, 22, 28-39.

Michie, S., \& Abraham, C. (2004). Interventions to change health behaviours: Evidence-based or evidence-inspired? Psychology and Health, 19, 29-49.

Miller, W. R. (2001). When is it motivational interviewing? Addiction, 96, 1770-1771.

Miller, W. R., \& Mount, K. A. (2001). A small study of training in motivational interviewing: does one workshop change clinician and client behavior? Behavioural and Cognitive Psychotherapy, 29, 457-471.

Miller, W. R., \& Rollnick, S. (2002). Motivational interviewing: preparing people for change (2nd ed.). New York, NY: Guilford.

Miller, W. R., \& Rose, G. S. (2009). Toward a theory of motivational interviewing. American Psychologist, 64, 527-537.

Miller, W. R., Yahne, C. E., Moyers, T. B., Martinez, J., \& Pirritano, M. (2004). A randomized trial of methods to help clinicians learn motivational interviewing. Journal of Consulting \& Clinical Psychology, 72, 1050-1062.

Morgan, D. (1988). Focus Groups as qualitative research. Qualitative research methods series 16 (Vol. 16). Beverly Hills: SAGE Publications Inc.

Nederlands Huisartsen Genootschap. (2006). NGH standaard diabetes mellitus type 2. Retrieved March 5, 2010, from http://nhg.artsennet.nl.

Nederlandse Diabetes Federatie. (2005). NDF Advies Diabeteseducatie. Retrieved June 18, 2009, from www.diabetesfederatie.nl.

Nederlandse Diabetes Federatie. (2007). NDF Zorgstandaard. Transparantie en kwaliteit van diabeteszorg voor mensen met diabetes type 2. Retrieved March 5, 2010, from www.diabetesfederatie.nl.

Nederlandse Diabetes Federatie. (2010). NAD contouren werkprogramma. Retrieved July 27, 2010, from http://www.actieprogrammadiabetes.nl/images/stories/ Contouren_werkprogramma_NAD.pdf

Nelson, K. M., Reiber, G., \& Boyko, E. J. (2002). Diet and exercise among adults with type 2 diabetes: findings from the third national health and nutrition examination survey (NHANES III). Diabetes Care, 25, 1722-1728. 
Newman, S., Steed, L., \& Mulligan, K. (2004). Self-management interventions for chronic illness. Lancet, 364, 1523-1537.

Norris, S. L., Engelgau, M. M., \& Narayan, K. M. (2001). Effectiveness of selfmanagement training in type 2 diabetes: a systematic review of randomized controlled trials. Diabetes Care, 24, 561-587.

Norris, S. L., Lau, J., Smith, S. J., Schmid, C. H., \& Engelgau, M. M. (2002). Selfmanagement education for adults with type 2 diabetes: a meta-analysis of the effect on glycemic control. Diabetes Care, 25, 1159-1171.

Puder, J. J., \& Keller, U. (2003). Quality of diabetes care: problem of patient or doctor adherence? Swiss Medical Weekly, 133, 530-534.

Rasbash, J., Steele, F., Browne, W., \& Prosser, B. (2005). A user's guide to Mlwin, version 2. Bristol: Centre for Multilevel Modelling, University of Bristol.

Renders, C. M., Valk, G. D., Griffin, S., Wagner, E. H., Eijk, J. T., \& Assendelft, W. J. (2001). Interventions to improve the management of diabetes mellitus in primary care, outpatient and community settings. Cochrane Database Systematic Review, Cd001481.

Resnick, H. E., Foster, G. L., Bardsley, J., \& Ratner, R. E. (2006). Achievement of American Diabetes Association clinical practice recommendations among U.S. adults with diabetes, 1999-2002: the National Health and Nutrition Examination Survey. Diabetes Care, 29, 531-537.

Resnicow, K., DiIorio, C., Soet, J. E., Ernst, D., Borrelli, B., \& Hecht, J. (2002). Motivational interviewing in health promotion: it sounds like something is changing. Health Psychology, 21, 444-451.

Richards, D. A., \& Hamers, J. P. (2009). RCTs in complex nursing interventions and laboratory experimental studies. International Journal of Nursing Studies, 46, 588592.

RIVM. (2010). Nationaal Kompas Volksgezondheid, versie 3.21. Retrieved April 13, 2010, from www.nationaalkompas.nl.

Rollnick, S. (1996). Behaviour change in practice: targeting individuals. International Journal of Obesity and Related Metabolic Disorders, 20, S22-26.

Rollnick, S. (2001). Enthusiasm, quick fixes and premature controlled trials. Addiction, 96, 1769-1770.

Rollnick, S., Mason, P., \& Butler, C. (1999). Health behavior change: a guide for practitioners. London: Churchill Livingstone.

Rossi, P., Freeman, H., \& Lipsey, M. (1999). Evaluation: a systematic approach. Thousand Oaks: Sage Publications.

Rubak, S., Sandbaek, A., Lauritzen, T., Borch Johnsen, K., \& Christensen, B. (2006). An education and training course in motivational interviewing influence: GPs' professional behaviour-ADDITION Denmark. British Journal of General Practice, 56, 429-436. 
Rubak, S., Sandbaek, A., Lauritzen, T., \& Christensen, B. (2005). Motivational interviewing: a systematic review and meta-analysis. British Journal of General Practice, 55, 305-312.

Rutten, G. E. H. M. (2010). Diabetes, van wetenschap naar praktijk. Assen: Van Gorcum.

Sacco, W. P., Malone, J. I., Morrison, A. D., Friedman, A., \& Wells, K. (2009). Effect of a brief, regular telephone intervention by paraprofessionals for type 2 diabetes. Journal of Behavioral Medicine, 32, 349-359.

Saunders, R. P., Evans, M. H., \& Joshi, P. (2005). Developing a process-evaluation plan for assessing health promotion program implementation: a how-to guide. Health Promotion Practice, 6, 134-147.

Semaan, S., Des Jarlais, D. C., Sogolow, E., Johnson, W. D., Hedges, L. V., Ramirez, G., Flores, S. A., Norman, L., Sweat, M. D., \& Needle, R. (2002). A meta-analysis of the effect of HIV prevention interventions on the sex behaviors of drug users in the United States. Journal of Acquired Immune Deficiency Syndromes, 30, S7393.

Silberg, W. M., Lundberg, G. D., \& Musacchio, R. A. (1997). Assessing, controlling, and assuring the quality of medical information on the Internet: caveant lector et viewor - Let the reader and viewer beware. Journal of the American Medical Association, 277, 1244-1245.

Skelly, A. H., Carlson, J. R., Leeman, J., Holditch Davis, D., \& Soward, A. C. (2005). Symptom-focused management for African American women with type 2 diabetes: a pilot study. Applied Nursing Research, 18, 213-220.

Smith-West, D., DiLillo, V., Bursac, Z., Gore, S. A., \& Greene, P. G. (2007). Motivational interviewing improves weight loss in women with type 2 diabetes. Diabetes Care, 30, 1081-1087.

Smith, D. E., Heckemeyer, C. M., Kratt, P. P., \& Mason, D. A. (1997). Motivational interviewing to improve adherence to a behavioral weight-control program for older obese women with NIDDM. A pilot study. Diabetes Care, 20, 52-54.

Snijders, T. A. B., \& Bosker, R. J. (1999). Multilevel analysis: an introduction to basic and advanced multilevel modeling. London: Sage Publications Ltd.

Snoek, F. J., \& Hogenelst, M. H. (2008). Psychologische implicaties van diabetes mellitus. Nederlands Tijdschrift voor Geneeskunde, 152, 2395-2399.

Snoek, F. J., Pouwer, F., Welch, G. W., \& Polonsky, W. H. (2000). Diabetes-related emotional distress in Dutch and U.S. diabetic patients: cross-cultural validity of the problem areas in diabetes scale. Diabetes Care, 23, 1305-1309.

Snoek, F. J., \& Skinner, T. C. (2005). Psychology in Diabetes Care. Chichester: Wiley.

Stampfer, M. J., Hu, F. B., Manson, J. E., Rimm, E. B., \& Willett, W. C. (2000). Primary prevention of coronary heart disease in women through diet and lifestyle. New England Journal of Medicine, 343, 16-22. 
Steckler, A., Goodman, R. M., McLeroy, K. R., Davis, S., \& Koch, G. (1992). Measuring the diffusion of innovative health promotion programs. American Journal of Health Promotion, 6, 214-224.

Steed, L., Lankester, J., Barnard, M., Earle, K., Hurel, S., \& Newman, S. (2005). Evaluation of the UCL diabetes self-management programme (UCL-DSMP): a randomized controlled trial. Journal of Health Psychology, 10, 261-276.

Stewart, M., Brown, J. B., Donner, A., McWhinney, I. R., Oates, J., Weston, W. W., \& Jordan, J. (2000). The impact of patient-centered care on outcomes. Journal of Family Practice, 49, 796-804.

Stichting Regionale Huisartsenzorg Heuvelland. (2010). Eerstelijns keten DBC diabetes Mellitus type 2, jaarverslag 2009. Maastricht: Stichting RHZ.

Stratton, I. M., Adler, A. I., Neil, H. A., Matthews, D. R., Manley, S. E., Cull, C. A., Hadden, D., Turner, R. C., \& Holman, R. R. (2000). Association of glycaemia with macrovascular and microvascular complications of type 2 diabetes (UKPDS 35): prospective observational study. British Medical Journal, 321, 405-412.

Street, R. L., Jr., Piziak, V. K., Carpentier, W. S., Herzog, J., Hejl, J., Skinner, G., \& McLellan, L. (1993). Provider-patient communication and metabolic control. Diabetes Care, 16, 714-721.

Suls, J., Martin, R., \& Wheeler, L. (2002). Social comparison: why, with whom, and with what effect? Current Directions in Psychological Science, 11, 159-163.

Surgenor, L. J., Horn, J., Hudson, S. M., Lunt, H., \& Tennent, J. (2000). Metabolic control and psychological sense of control in women with diabetes mellitus. Alternative considerations of the relationship. Journal of Psychosomatic Research, 49, 267-273.

Tomky, D., Cypress, M., Dang, D., Maryniuk, M., \& Peyrot, M. (2008). AADE7TM Self-care behaviors. The Diabetes Educator, 34, 445-449.

Toobert, D. J., Glasgow, R. E., Strycker, L. A., Barrera, M., Jr., Radcliffe, J. L., Wander, R. C., \& Bagdade, J. D. (2003). Biologic and quality-of-life outcomes from the Mediterranean Lifestyle Program: a randomized clinical trial. Diabetes Care, 26, 2288-2293.

Toobert, D. J., Glasgow, R. E., Strycker, L. A., Barrera, M., Ritzwoller, D. P., \& Weidner, G. (2007). Long-term effects of the Mediterranean Lifestyle Program: a randomized clinical trial for postmenopausal women with type 2 diabetes. International Journal of Behavioral Nutrition and Physical Activity, 4, 1.

Toobert, D. J., Hampson, S. E., \& Glasgow, R. E. (2000). The summary of diabetes self-care activities measure: results from 7 studies and a revised scale. Diabetes Care, 23, 943-950.

Toobert, D. J., Strycker, L. A., Glasgow, R. E., Barrera, M., \& Angell, K. (2005). Effects of the Mediterranean Lifestyle Program on multiple risk behaviors and psychosocial outcomes among women at risk for heart disease. Annals of Behavioral Medicine, 29, 128-137. 
UK Prospective Diabetes Study Group. (1998). Intensive blood-glucose control with sulphonylureas or insulin compared with conventional treatment and risk of complications in patients with type 2 diabetes (UKPDS 33). Lancet, 352, 837-853.

Van Assema, P., Brug, J., Ronda, G., \& Steenhuis, I. (2001). The relative validity of a short Dutch questionnaire as a means to categorize adults and adolescents to total and saturated fat intake. Journal of Human Nutrition and Dietetics, 14, 377-390.

Van Assema, P., Brug, J., Ronda, G., Steenhuis, I., \& Oenema, A. (2002). A short Dutch questionnaire to measure fruit and vegetable intake: relative validity among adults and adolescents. Nutrition Health, 16, 85-106.

Van Assema, P., Mesters, I., \& Kok, G. (1992). Het focusgroep-interview: een stappenplan. Tijdschrift voor gezondheidswetenschappen, 70, 431-437.

Van Dam, H. A., Van der Horst, F., Van den Borne, B., Ryckman, R., \& Crebolder, H. (2003). Provider-patient interaction in diabetes care: effects on patient self-care and outcomes. A systematic review. Patient Education and Counseling, 51, 17-28.

Van den Arend, I. J. M., Stolk, R. P., Krans, H. M. J., Grobbee, D. E., \& Schrijvers, A. J. P. (2000). Management of type 2 diabetes: a challenge for patient and physician. Patient Education and Counseling, 40, 187-194.

Van der Bijl, J., Van Poelgeest-Eeltink, A., \& Shortridge-Baggett, L. M. (1999). The psychometric properties of the diabetes management self-efficacy scale for patients with type 2 diabetes mellitus. Journal of Advanced Nursing, 30, 352-359.

Van Keulen, H. M., Mesters, I., Brug, J., Ausems, M., Campbell, M., Resnicow, K., Zwietering, P. J., Van Breukelen, G., Van Mechelen, W., Severens, J. L., \& De Vries, H. (2008). Vitalum study design: RCT evaluating the efficacy of tailored print communication and telephone motivational interviewing on multiple health behaviors. BioMed Central Public Health, 8, 216.

VanWormer, J. J., \& Boucher, J. L. (2004). Motivational interviewing and diet modification: a review of the evidence. The Diabetes Educator, 30, 404-419.

Verhoeven, F., Tanja-Dijkstra, K., Nijland, N., Eysenbach, G., \& van Gemert-Pijnen, L. (2010). Asynchronous and synchronous teleconsultation for diabetes care: a systematic literature review. Journal of Diabetes Science and Technology, 4, 666684.

Vermeire, E., Wens, J., Van Royen, P., Biot, Y., Hearnshaw, H., \& Lindenmeyer, A. (2005a). Interventions for improving adherence to treatment recommendations in people with type 2 diabetes mellitus. Cochrane Database of Systematic Reviews, Art. No.: CD003638. DOI:003610.001002/14651858.CD14003638.pub14651852.

Vermeire, E., Wens, J., Van Royen, P., Biot, Y., Hearnshaw, H., \& Lindenmeyer, A. (2005b). Interventions for improving adherence to treatment recommendations in people with type 2 diabetes mellitus. Cochrane Database of Systematic Reviews, Art. No.: CD003638. DOI:003610.001002/14651858.CD14003638.pub14651852.

Vrijhoef, H. J., Diederiks, J. P., Spreeuwenberg, C., \& Wolffenbuttel, B. H. (2001). Substitution model with central role for nurse specialist is justified in the care for stable type 2 diabetic outpatients. Journal of Advanced Nursing, 36, 546-555. 
Wagner, E. H., Austin, B. T., Davis, C., Hindmarsh, M., Schaefer, J., \& Bonomi, A. (2001). Improving chronic illness care: translating evidence into action. Health Affairs, 20, 64-78.

Wagner, E. H., Austing, B. T., \& Von Korff, M. (1996). Organizing care for patients with chronic illness. The Milbank Quarterly, 511-544.

Wagner, E. H., Bennett, S. M., Austin, B. T., Greene, S. M., Schaefer, J. K., \& Vonkorff, M. (2005). Finding common ground: patient-centeredness and evidencebased chronic illness care. Journal of Alternative and Complementary Medicine, $11, \mathrm{~S} 7-15$.

Wallston, K. A., Stein, M. J., \& Smith, C. A. (1994). Form C of the MHLC scales: a condition-specific measure of locus of control. Journal of Personality Assessment, 63, 534-553.

Wallston, K. A., Wallston, B. S., \& DeVellis, R. (1978). Development of the Multidimensional Health Locus of Control (MHLC) Scales. Health Education Monographs, 6, 160-170.

Walters, S. T., Matson, S. A., Baer, J. S., \& Ziedonis, D. M. (2005). Effectiveness of workshop training for psychosocial addiction treatments: a systematic review. Journal of Substance Abuse Treatment, 29, 283-293.

Watkins, K. W., Connell, C. M., Fitzgerald, J. T., Klem, L., Hickey, T., \& Ingersoll Dayton, B. (2000). Effect of adults' self-regulation of diabetes on quality-of-life outcomes. Diabetes Care, 23, 1511-1515.

Wattana, C., Srisuphan, W., Pothiban, L., \& Upchurch, S. L. (2007). Effects of a diabetes self-management program on glycemic control, coronary heart disease risk, and quality of life among Thai patients with type 2 diabetes. Nursing \& Health Sciences, 9, 135-141.

Wens, J., Vermeire, E., Hearnshaw, H., Lindenmeyer, A., Biot, Y., \& Van Royen, P. (2008). Educational interventions aiming at improving adherence to treatment recommendations in type 2 diabetes: a sub-analysis of a systematic review of randomised controlled trials. Diabetes Research and Clinical Practice, 79, 377-388.

Whittemore, R., Melkus, G. D., Sullivan, A., \& Grey, M. (2004). A nurse-coaching intervention for women with type 2 diabetes. The Diabetes Educator, 30, 795-804.

WHO. (2009). Global health risks: mortality and burden of disease attributable to selected major risks. Geneva: World Health Organization.

Wild, S., Roglic, G., Green, A., Sicree, R., \& King, H. (2004). Global Prevalence of Diabetes. Estimates for the year 2000 and projections for 2030. Diabetes Care, 27, 1047-1053.

Williams, G. C., Freedman, Z. R., \& Deci, E. L. (1998). Supporting autonomy to motivate patients with diabetes for glucose control. Diabetes Care, 21, 1644-1651.

Williams, G. C., Grow, V. M., Freedman, Z. R., Ryan, R. M., \& Deci, E. L. (1996). Motivational predictors of weight loss and weight-loss maintenance. Journal of Personality and Social Psychology, 70, 115-126. 
Williams, G. C., McGregor, H., Zeldman, A., Freedman, Z. R., Deci, E. L., \& Elder, D. (2005). Promoting glycemic control through diabetes self-management: evaluating a patient activation intervention. Patient Education and Counseling, 56, 28-34.

Williams, G. C., McGregor, H. A., Zeldman, A., Freedman, Z. R., \& Deci, E. L. (2004). Testing a self-determination theory process model for promoting glycemic control through diabetes self-management. Health Psychology, 23, 58-66.

Williams, G. C., Rodin, G. C., Ryan, R. M., Grolnick, W. S., \& Deci, E. L. (1998). Autonomous regulation and long-term medication adherence in adult outpatients. Health Psychology, 17, 269-276.

Windsor, R., Baranowski, T., Clark, N., \& Cutter, G. (1994). Evaluation of health promotion, health education and disease prevention programs. Mountain View: Mayfield.

Wyatt, J. C. (1997). Commentary: measuring quality and impact of the World Wide Web. British Medical Journal, 314, 1879-1881. 
150 
Dankwoord 
152 
Dit proefschrift zou niet tot stand zijn gekomen zonder de medewerking van zorgverleners en mensen met diabetes. Allereerst bedank ik daarom al deze mensen.

Mijn begeleiders Nanne de Vries en Nicolaas Schaper bedank ik voor de kans die ze mij gaven om dit project uit te voeren en voor het delen van hun expertise.

Bedankt mensen van Stichting DIEP en diep@work voor de prettige samenwerking en Els Denis in het bijzonder voor haar hulp met name gedurende de eerste fasen van het project.

Mijn oud-collegae van de afdeling Gezondheidsbevordering wil ik bedanken voor het uitwisselen van kennis en ervaring, voor de steun bij het afronden van mijn promotie en voor de gezelligheid.

Judith en Froukje, zonder jullie als paranimfen was alles anders geweest. Bedankt voor de hulp bij de laatste loodjes van het promoveren en voor alles wat daaraan vooraf ging.

Lieve familie en vrienden, bedankt voor jullie interesse in mijn werk, voor het soms juist zwijgen over mijn proefschrift, voor het sturen van bemoedigende kaartjes en e-mails en natuurlijk voor de afleiding en de leuke momenten samen.

Leon, wat ben ik blij dat ik je heb leren kennen! De toekomst met jou te mogen delen voelt geweldig. Bedankt voor alles dat je me geeft en vooral ook voor je positieve instelling en je vertrouwen in mijn kunnen!

\section{Evelien}


154 
Curriculum Vitae 
156 


\begin{abstract}
About the author
Evelien Heinrich was born on September 23, 1981 in Enschede, the Netherlands. In 1999 she received her VWO diploma at 'Het Assink' in Haaksbergen. That same year, she started the academic studies Health Education and Health Promotion at the Faculty of Health Sciences of the Maastricht University, from which she graduated in August 2003. Evelien subsequently worked for one year as a research assistant on a project on smoking prevention and smoking cessation among 15-17 year-old Dutch adolescents at the Department of Health Promotion. In 2004, she continued her carreer at the same department as a $\mathrm{PhD}$ student on the project 'Diabetes education in primary care: improving self-management'. The studies she conducted resulted in the current dissertation. Besides her $\mathrm{PhD}$ project, she engaged in 'Stichting DIEP' as a researcher and as a member of the editorial staff.

In July 2010, Evelien was appointed leader of a project on care for the elderly at Health Centre 'Withuis' in Venlo. From September 2010 she combines this job with a position as primary care consultant for the regional support organisation 'Stichting Fast' in Eindhoven.
\end{abstract}


